\title{
Evidence-Based Guideline of the German Nutrition Society: Carbohydrate Intake and Prevention of Nutrition-Related Diseases
}

\author{
Hans Hauner $^{\mathrm{a}} \quad$ Angela Bechthold $^{\mathrm{b}}$ Heiner Boeing ${ }^{c} \quad$ Anja Brönstrup $^{\mathrm{b}}$ \\ Anette Buyken $^{d}$ Eva Leschik-Bonnet ${ }^{b}$ Jakob Linseisen ${ }^{e}$ Matthias Schulze ${ }^{c}$ \\ Daniela Strohm $^{\text {b }}$ Günther Wolfram ${ }^{a}$ \\ ${ }^{a}$ Technical University of Munich, Freising-Weihenstephan, ${ }^{b}$ German Nutrition Society, Bonn, ${ }^{c}$ German Institute of \\ Human Nutrition, Potsdam-Rehbrücke, d Research Institute of Child Nutrition, Dortmund, and ${ }^{\mathrm{e} H e l m h o l t z ~ Z e n t r u m, ~}$ \\ Munich, Germany
}

\section{Key Words}

Carbohydrates $\cdot$ Obesity $\cdot$ Type 2 diabetes $\cdot$ Coronary heart disease $\cdot$ Dyslipoproteinaemia $\cdot$ Hypertension $\cdot$ Metabolic syndrome $\cdot$ Cancer $\cdot$ Prevention

\begin{abstract}
The relative contribution of nutrition-related chronic diseases to the total disease burden of the society and the health care costs has risen continuously over the last decades. Thus, there is an urgent necessity to better exploit the potential of dietary prevention of diseases. Carbohydrates play a major role in human nutrition - next to fat, carbohydrates are the second biggest group of energy-yielding nutrients. Obesity, type 2 diabetes mellitus, dyslipoproteinaemia, hypertension, metabolic syndrome, coronary heart disease and cancer are wide-spread diseases, in which carbohydrates could have a pathophysiologic relevance. Correspondingly, modification of carbohydrate intake could have a preventive potential. In the present evidence-based guideline of the German Nutrition Society, the potential role of carbohydrates in the primary prevention of the named diseases was judged systematically. The major findings were: a high carbohydrate intake at the expense of total fat and saturated fatty acids reduces the concentrations of total, LDL and HDL cholester-
\end{abstract}

ol. A high carbohydrate consumption at the expense of polyunsaturated fatty acids increases total and LDL cholesterol, but reduces HDL cholesterol. Regardless of the type of fat being replaced, a high carbohydrate intake promotes an increase in the triglyceride concentration. Furthermore, a high consumption of sugar-sweetened beverages increases the risk of obesity and type 2 diabetes mellitus, whereas a high dietary fibre intake, mainly from whole-grain products, reduces the risk of obesity, type 2 diabetes mellitus, dyslipoproteinaemia, cardiovascular disease and colorectal cancer at varying evidence levels. The practical consequences for current dietary recommendations are presented.

Copyright $\odot 2012$ S. Karger AG, Basel

\section{Introduction}

It is well known that nutrition has a substantial influence on the development and progression of many chronic diseases. These include especially obesity, type 2 diabetes mellitus, dyslipoproteinaemia, hypertension, heart and vascular diseases and cancer. In recent decades, the relative importance of these diseases has continually increased; correspondingly, their part of the expenditure of the health care system has grown. In addition, new studies

\section{KARGER \\ Fax +41613061234 E-Mail karger@karger.ch} www.karger.com

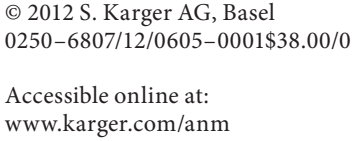

\footnotetext{
Dr. Daniela Strohm

Department of Science, German Nutrition Society

Godesberger Allee 18

DE-53175 Bonn (Germany)

Tel. +49 2283776 623,E-Mail strohm@dge.de
} 
indicate that there is a very high potential to prevent these diseases, and this has not yet been fully exploited [WHO, 2003, 2009].

According to its constitution, the German Nutrition Society (DGE) aims to improve the health of the general public by developing and communicating scientifically based nutritional recommendations which contribute to the primary prevention of nutrition-related diseases. Already in 2006, with this intention a Guideline Commission published an evidence-based guideline regarding fat intake and prevention of nutrition-related diseases, which is publicly available (www.dge.de/leitlinie).

Next to fat, carbohydrates are the second big group of energy-yielding nutrients, providing an important part of human nutrition. Correspondingly, they could have a substantial influence on the prevention of nutrition-related diseases. In the context of systematically investigating macronutrients regarding their preventive potential, a Guideline Commission of the DGE has addressed the question of the role of carbohydrate intake on the occurrence of nutrition-related diseases in healthy individuals [DGE, 2011a]. For this purpose, the current knowledge based on human studies was critically reviewed, and scientifically validated information was extracted. The results of this evidence-based guideline should contribute to reduce consumer's uncertainty which is caused by contradictory statements regarding the influence of nutritional carbohydrate intake. Especially in recent years, there has been intensive and controversial discussion about this [Mack and Hauner, 2007].

The aim of this evidence-based guideline regarding carbohydrate intake and the prevention of certain nutrition-related diseases is:

- to perform a systematic review of the scientific literature on the effects of carbohydrate intake on the development and prevention of diseases;

- to separately assess single qualitative parameters of carbohydrate intake in this context;

- to provide scientifically based information on the effects of carbohydrate quantity and quality for nutrition experts and the media.

Because of the clinical significance of nutrition-related diseases and their relevance in preventive medicine, this systematic review focussed on obesity, type 2 diabetes mellitus, dyslipoproteinaemia, hypertension, metabolic syndrome, coronary heart disease (CHD) and cancer. Due to limited rescources, it was not possible to include all diseases for which an impact of carbohydrate intake has been suggested, like gastrointestinal diseases and dental caries.

\section{Methodological Approach}

\subsection{Key Questions}

The key questions were: does the quantity and quality of carbohydrate intake have an impact on the development of nutritionrelated diseases like obesity, type 2 diabetes mellitus, dyslipoproteinaemia, hypertension, metabolic syndrome, CHD and cancer? If so, of which directions are the observed effects and how strong is the impact of carbohydrate intake? Last but not least, what are the resulting practical recommendations for primary prevention?

First, the aspects of carbohydrate intake to be investigated were identified. The Guideline Commission agreed on the following to be considered in the present guideline:

- total carbohydrates (percentage of energy intake or absolute amount)

- mono- and disaccharides (sugar), sugar-sweetened beverages

- polysaccharides

- dietary fibre/whole-grain products

- glycaemic index (GI) and glycaemic load (GL)

The Guideline Commission has chosen this selection due to the relevance of these aspects and in order to cover important prevention potentials of carbohydrate intake. In addition, sufficient study data are available for these aspects.

\subsection{Search Strategy}

A systematic literature search was performed regarding the key questions. The focus was on meta-analyses, systematic reviews and original papers published between 1975 and December 2009. Meta-analyses from 2010 published before December 15, 2010, were also included.

The search was conducted explicitly for human studies in English or German language. The literature search was performed using the PubMed database (www.ncbi.nlm.nih.gov/pubmed) and reviewing the reference lists of guidelines, reviews and original papers.

\subsection{Classification of the Literature into Levels of Evidence}

The classification of the levels of evidence which evaluate the study design and results with regard to their potential relevance was conducted according to the judgement scheme of the World Health Organization (WHO) [WHO, 2003]. The judgement of the selected literature was performed on the basis of the levels of evidence shown in table 1.

The focus of the judgement was put on randomised controlled intervention studies ${ }^{1}$ with the best evidence (level of evidence I) and prospective cohort studies with the second best evidence (level of evidence II). Case-control studies (level of evidence III) and non-analytic studies (level of evidence IV) were not included. In case-control studies, it is principally possible to use detailed assessment instruments; however, this advantage does not outweigh the methodological weakness of the 'wrong' chronology of exposure and disease occurrence and the associated sources of bias. Cross-sectional studies were not considered for this guideline either, because they do not allow statements regarding chronology (risk of reverse causation), and because existing diseases possibly lead to bias in the nutritional assessment.

1 The present DGE guideline includes mainly intervention studies with a duration of at least 12 weeks. 
Table 1. Classification of levels of evidence (LOE)

\section{LOE I}

Ia Meta-analysis of randomised controlled intervention studies

Ib Randomised controlled intervention studies

Ic Non-randomised/non-controlled intervention studies (if well-designed)

LOE II

IIa Meta-analysis of cohort studies

IIb Cohort studies

\section{LOE III}

IIIa Meta-analysis of case-control studies

IIIb Case-control studies

\section{LOE IV}

IV Non-analytic studies (cross-sectional studies, case reports etc.) as well as reports/opinions of expert committees or consensus conferences, where no strength of evidence was stated, and/or clinical experience of respected authorities

Characteristics and results of all studies fully meeting the inclusion criteria were systematically recorded (see tables for each health outcome: http://www.dge.de/leitlinie; German only). Figure 1 gives an overview of the number of identified publications.

\subsection{Judgement of the Strength of the Evidence}

For evidence judgement, the strength of the evidence was rated, taking into account the scientific evidence (level of evidence, LOE) of the single studies. The strength of the evidence indicates the respective quality and consistency of the scientific data upon which a statement is based. Following the judgement scheme of the WHO [WHO, 2003], the strength of the evidence was judged as 'convincing', 'probable', 'possible' and 'insufficient' regarding a preventive effect or a lack of an association (table 2).

The chronology of associations (cohort studies) as well as results from intervention studies and the biological plausibility were included into the judgement of the strength of the evidence. As a consequence, the strength of the evidence also provides information on causation and justifies the deviation of recommendations for primary prevention on a population level.

Based upon the performed judgement of the evidence, foodbased recommendations for carbohydrate intake were derived. The guideline draft was presented online for 2 months for discussion and comments. After taking into consideration relevant comments received during this period, the guideline was published.

\subsection{Studies on Primary and Secondary Prevention}

In principle, it has to be considered in the interpretation of study data and the resulting prevention measures or recommendations if the studies were performed on primary or on secondary prevention. The topic of primary preventive research is to avoid triggering or present causes or partial causes of a disease with the aim to lower the incidence of the disease in the entire population or the risk of occurrence in an individual [Walter and Schwartz,
2003]. Accordingly, study subjects are not yet suffering from the disease that is investigated. In contrast, secondary prevention deals with the early therapy of existing diseases and aims to lower the incidence of manifest or progressed diseases in general. This also includes the prevention of recurring events (like reinfarction) and the avoidance of secondary diseases. Thus, in this case the study population consists of patients with a corresponding medical history. The present guideline only includes studies on primary prevention.

\subsection{Quality and Type of Data on Carbohydrate Intake}

Like other energy-yielding nutrients, carbohydrate intake is closely associated with energy intake. This means that individuals with higher energy intake usually ingest more carbohydrates than individuals with lower energy intake. Most of the variation between individuals regarding carbohydrate intake is therefore due to differences in total energy intake. However, it was neither the focus of interest if people have a high or low energy intake (and, correspondingly, a high or low carbohydrate uptake), nor if they are of different gender, weight or more or less physically active, but rather if they have a high or low carbohydrate intake in relation to similar individuals. In observational studies, risk factors can be statistically adjusted between the investigated groups.

There are often no intervention studies assessing the effects of carbohydrate intake on the prevention of certain nutrition-related diseases. For obesity, most of the studies identified were studies on weight reduction, which have not been considered relevant for the present question (see chapter 3). In addition, heterogeneity between studies regarding the definition of endpoints has to be taken into account. For example, for the definition of the metabolic syndrome (see chapter 7), different levels of elevated fasting blood glucose levels have been considered, which in part may explain the different results observed.

Nutritional interventions differ from medical therapeutic interventions in some important features [Knorpp and Kroke, 2011]. In the field of nutrition, conduction of randomised controlled intervention studies is often not possible, due to ethical and other reasons. Even if they were ethically justifiable, there is still the problem that nutritional interventions cannot be blinded. Because of this, knowledge on the relation between nutritional factors and diseases is often derived from observational studies like the ones that were mainly used for the present guideline, and not from randomised controlled intervention studies [Kroke et al., 2003]. In addition, nutritional interventions most often have very complex effects. For example, individual nutrient intake and nutrition on the whole are influenced in many different ways by changing the intake of a single nutrient [Knorpp and Kroke, 2011]. Hence, only focussing on randomised controlled intervention studies is not appropriate for deriving nutritional recommendations.

The aim of this research was to identify studies on total carbohydrate intake or on the intake of certain kinds of carbohydrates, respectively. Therefore, studies that considered carbohydrate-containing foods like bread, pasta or potatoes as exposure factors were not included into the evaluation. One exception was the group of sugar-sweetened beverages. Sugar-sweetened beverages are defined as carbonated soft drinks like cola beverages and lemonades as well as non-carbonated soft drinks like beverages with fruit juice or nectar and ice tea that contain added sugar. Unsweetened fruit juices are not included. In North America, 


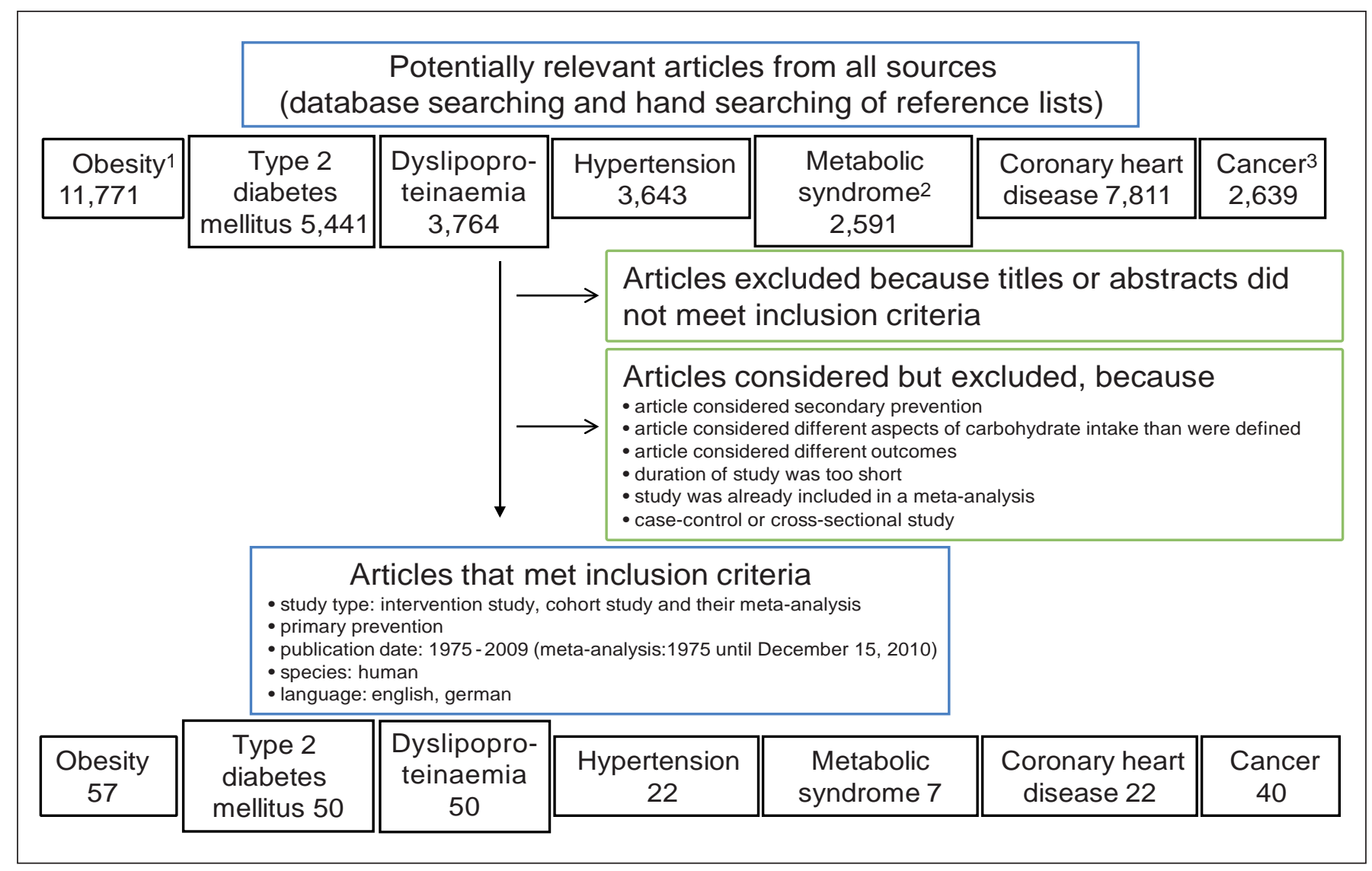

Fig. 1. PRISMA flow chart of studies identified, screened and excluded or included during the literature search and review. ${ }^{1}$ Original papers published from 2006; for earlier papers we referred to the literature search for 'obesity' which was enclosed in the report of the World Cancer Research Fund [International Journal of Obe- sity, 2009]. ${ }^{2}$ Original papers published from 2001 where 'metabolic syndrome' was defined according to the International Diabetes Federation [Alberti et al., 2005]. ${ }^{3}$ Original papers published from 2005, for earlier papers we referred to the literature search of the World Cancer Research Fund [WCRF, 2007]. these beverages are usually sweetened with high-fructose corn syrup (HFCS) with a fructose content of 42 or $55 \%$, while in Europe, sucrose is usually added as sweetener [Jones, 2009]. The energy content of these beverages almost exclusively depends on their content of mono- and disaccharides. Therefore, the results of these studies are presented in the subparagraphs mono- and disaccharides in the respective chapters. As another exception, studies on whole-grain products or refined grain products, respectively, were included into the research, since considering these food groups as exposure factor indirectly allows statements on the influence of cereal dietary fibre, and there are a number of studies available regarding this aspect.

\subsection{Search Terms Used for the Search Strategy}

For the search in PubMed, the following search terms were used for the different health outcomes considered in this guideline:

Obesity: obesity, overweight, weight gain, BMI, body mass index, waist circumference, weight maintenance, caloric intake, body fat, energy intake.
Type 2 diabetes mellitus: diabetes, insulin resistance, insulin sensitivity, prediabetes, impaired glucose tolerance, impaired fasting glucose, fructosamine, A1c.

Dyslipoproteinaemia: dyslipoprotein(a)emia, hyperlipoprotein(a)emia, hyperlipid(a)emia, hypercholesterol(a)emia, hypertriglycerid(a)emia, dyslipid(a)emia, serum/plasma triglycerides, plasma lipids, serum/plasma cholesterol, LDL, HDL, lipoprotein(s), risk factor(s) + cardiovascular.

Hypertension: hypertension, blood pressure, hypertonia.

Metabolic syndrome: metabolic syndrome, syndrome X.

Coronary heart disease (CHD): cardiovascular disease, CVD, coronary artery disease, coronary heart disease, CHD, myocardial infarction, arteriosclerosis, atherosclerosis.

Cancer: cancer, polyp(s), adenoma, tumor.

The search terms for the different health outcomes each were connected to the following search terms: carbohydrate, fibre/fiber, whole grain(s), refined grain(s), sugar(s), sucrose, fructose, glucose, starch, glyc(a)emic, monosaccharide(s), disaccharide(s), polysaccharide(s), (macro)nutrient(s). 
Table 2. Strength of the evidence

\begin{tabular}{ll}
$\begin{array}{l}\text { Strength of } \\
\text { the evidence }\end{array}$ & $\begin{array}{l}\text { Criteria for strength of the evidence } \\
\text { Convincing }\end{array}$ \\
$\begin{array}{l}\text { There are a considerable number of studies including prospective cohort studies and, where possible, randomised } \\
\text { controlled intervention studies of sufficient size, duration and quality with consistent results (at least } 2 \text { randomised } \\
\text { controlled intervention studies of the highest quality (LOE I); in case of methodological weakness or cohort studies } \\
\text { only: at least 5). Ideally a meta-analysis of the present studies is available (no heterogeneous study results, no high } \\
\text { percentage of study results with opposite effects). }\end{array}$ \\
\hline $\begin{array}{l}\text { Probable } \\
\text { weaknesses regarding the causal argumentation or there is evidence for an opposite relation, which does not allow } \\
\text { a definite judgement. The required number of studies for classifying the strength of the evidence as 'probable' } \\
\text { remains at as many as } 5 \text { very good studies (LOE I and/or LOE II). }\end{array}$ \\
$\begin{array}{l}\text { There are only intervention studies with high risk of bias, cohort studies or non-controlled clinical trials. } \\
\text { The results of the majority of the present studies and at least of } 3 \text { studies point in the same direction. } \\
\text { There may exist other studies without risk relations or with opposite risk relations, respectively. }\end{array}$ \\
$\begin{array}{l}\text { A few study results indicate an association between a factor and a disease, but they are not sufficient to establish the } \\
\text { relation. This means, the relation between nutritional factor and disease has not yet or rarely been investigated, or } \\
\text { the studies available are inconsistent with a majority of studies without risk relations and nearly equally as strong } \\
\text { opposite results. }\end{array}$ \\
\hline
\end{tabular}

If it was required to further limit the number of hits, this was performed by entering the following terms: intake, uptake, ingestion, consumption, prevention, nutrition(al), diet, dietary.

\section{Quantity and Quality of Carbohydrate Intake and Primary Prevention of Obesity}

There are only a few intervention studies upon which the evidence judgement regarding the prevention of obesity by carbohydrate intake can be based, because most of the intervention studies are therapeutic studies on weight reduction or weight maintenance, respectively, in individuals with already existing obesity. Though cohort studies, which are investigating the relation between food intake at baseline and the subsequent body weight development, allow conclusions concerning the cause-effect relation, they do not include possible changes of eating behaviour during follow-up. In particular, this is a problem regarding the endpoint development of obesity, as individuals who gain weight often change their food intake specifically to counteract weight gain. In part, this adaptation of behaviour is considered in cohort studies which relate nutritional changes to the simultaneous change of body composition. These approaches, however, do not allow conclusions on chronology, as cause and effect variables are measured repeatedly, yet stimultaneously [van Dam and Seidell, 2007].

\subsection{Proportion of Carbohydrates}

Observational studies on the proportion of carbohydrates examined the intake of carbohydrates as both percentage of energy uptake (EN\%) and in g/day. As eating habits in Europe differ from those in the USA, Canada or Australia, at first, results from European studies are presented. Cohort studies on children and adolescents are summarised by the age of the investigated collective. Details on the studies reviewed herein are presented in the tables for each health outcome (http://www.dge.de/leitlinie; German only).

\section{Adults}

Among 1,762 participants of the Danish MONICA arm, in the total population no association between carbohydrate intake and subsequent body weight development over 5 years could be detected. However, in men who were obese at baseline, higher carbohydrate intake $(\mathrm{EN} \%)$ was associated with greater weight reduction $[\beta$ $(\mathrm{SE})=-0.2(0.9) \mathrm{kg} / \mathrm{EN} \%$ per 5 years in men with BMI $<25 ;-1.0(1.0) \mathrm{kg} / \mathrm{EN} \%$ per 5 years in men with BMI $\geq 25$ to $<29.9 ;-4.7(2.7) \mathrm{kg} / \mathrm{EN} \%$ per 5 years in men with BMI $\geq 30 ; \mathrm{p}=0.03$ for interaction] [Iqbal et al., 2006] (LOE IIb). In the Danish Diet, Cancer and Health Study including about 45,000 women and men, no association was shown between carbohydrate intake at baseline and 
changes in waist circumference in the following 5 years [Halkjaer et al., 2006] (LOE IIb). Furthermore, in 288 healthy Dutch men, an increase in carbohydrate intake was not associated with simultaneous changes in either body weight or waist circumference over 5 years [Nooyens et al., 2005] (LOE IIb). In a cohort of 641 healthy US-American women and men, who were examined every 3 months over 1 year, baseline carbohydrate intake was not associated with subsequent changes in BMI [Ma et al., 2005] (LOE IIb). In a study by Kleges et al. [1992] (LOE IIb), involving 250 US-American men and women, the authors found no association between carbohydrate intake at baseline and change in body weight at the 1-year follow-up either. Likewise, in 465 US-American women and men, no association between baseline carbohydrate intake and changes in BMI during a 4-year follow-up could be observed [Parker et al., 1997] (LOE IIb). However, Colditz et al. [1990] (LOE IIb) showed in an investigation of 31,940 subjects in the Nurses' Health Study that higher carbohydrate intake at baseline was associated with a lower increase in body weight over 4 years. Likewise, 2,909 participants of the CARDIA study with a higher carbohydrate intake at baseline had a lower body weight after 10 years than those with lower carbohydrate intake (Caucasian women and men: quintile $1: 76.6 \mathrm{~kg}$ vs. quintile $5: 75.2 \mathrm{~kg}, \mathrm{p}=0.04$; Afro-Americans: quintile 1 : $84.1 \mathrm{~kg}$ vs. quintile $5: 82.6 \mathrm{~kg}, \mathrm{p}=0.03$ ). However, no prospective association with the waist-to-hip ratio was observed [Ludwig et al., 1999] (LOE IIb).

There are no targeted intervention studies on the preventive effect of increasing or decreasing the carbohydrate proportion in a diet. However, as lowering fat intake usually is accompanied by an increased intake of carbohydrates and dietary fibre, intervention studies on decreasing fat intake can indirectly provide information.

In the Women's Health Initiative Dietary Modification Trial (WHI) including 48,835 post-menopausal women (mean BMI 29), subjects in the intervention arm were motivated to reduce their fat intake and to increase their intake of fruits, vegetables and cereal products without any specific training on weight reduction. Among other aspects, this intervention resulted in an increased proportion of carbohydrate and dietary fibre $(52.7 \mathrm{EN} \%$ carbohydrates and $16.9 \mathrm{~g}$ dietary fibre vs. $44.7 \mathrm{EN} \%$ carbohydrates and $14.4 \mathrm{~g}$ dietary fibre in the control group) and led to an initial reduction in body weight compared with the control group $(-2.2 \mathrm{~kg}$ after year $1 ; \mathrm{p}<0.001)$ [Howard et al., 2006] (LOE Ib). In spite of rebound, body weight was still lower in the intervention group than in the con- trol group, even after 9 years $(-0.5 \mathrm{~kg} ; \mathrm{p}=0.001)$. After the initial weight loss, body weight increased steadily in the intervention group (comparable to the weight development in the control group). That means that the diet modification was able to delay weight gain for about 2 years, but could not prevent long-term weight gain. The beneficial effects observed may in particular be due to the simultaneous increase in dietary fibre intake. According to a multivariate secondary analysis, weight reduction was indeed associated with a reduction in fat intake and an increase in dietary fibre intake [Howard et al., 2006] (LOE IIb).

Fat-reduced ad libitum diet (26 EN\% fat, 54 EN\% carbohydrates) in an intervention with fibrocystic breast disease as an endpoint including 29 women with normal body weight resulted in a significantly reduced body weight after 6 months, without any specific training on weight reduction. In the control group, body weight remained constant (36 EN\% fat, 44 EN\% carbohydrates). However, after 1 year, the loss of weight was not significant anymore [Lee-Han et al., 1988] (LOE Ib). Details about the dietary fibre content of the diets were not available.

In 2 other intervention studies without the aim of weight reduction, basically normal-weight subjects were investigated, and no modification of carbohydrate intake was planned as intervention. In these studies, post hoc analyses on the influence of carbohydrate intake were performed: in a 1-year pilot study on the prevention of obesity including 40 young women with familial predisposition to obesity, change in nutritional carbohydrate proportion was not associated with the weight reduction that was achieved during the intervention [Eiben and Lissner, 2006] (LOE IIb). Lin et al. [2000] (LOE IIb) analysed data of a 2-year physical activity intervention study with 54 normal-weight US-American women on the influence of calcium on the development of body composition. Pooled analysis of the follow-up data after 2 years showed that higher carbohydrate intake (mean value of 5 surveys in 2 years) was associated with an increase in body weight and body fat percentage after 2 years (coefficient of correlation $r=0.33$ and 0.35 , respectively; $p<$ 0.05).

The available studies regarding adults mainly suggest that carbohydrate intake or dietary carbohydrate proportion, respectively, is not associated with the risk of obesity. The evidence regarding the lack of a long-term effect of a change in carbohydrate intake on the development of obesity is judged as probable. 
Children and Adolescents

In the Amsterdam Growth and Health Study (AGHS) including 181 13-year-old Dutch adolescents, carbohydrate intake at baseline was not associated with BMI or the sum of the skinfold thickness, respectively, after 15 years of follow-up [Twisk et al., 1998] (LOE IIb). In a second evaluation of this cohort, no association was found between carbohydrate intake and waist circumference after 15 years. However, in female adolescents an increase in carbohydrate intake was associated with an increase in subscapular skinfold thickness $[\beta=0.09$ (95\% CI 0.02; 0.16)] [van Lenthe et al., 1998] (LOE IIb). In a cohort of 509 12-year-old Northern Irish adolescents, baseline carbohydrate intake was not associated with a change in BMI or the sum of the skinfold thickness in the following 3 years [Boreham et al., 1999] (LOE IIb). In a group of overweight US-American adolescents of Latin-American origin $(n=85)$, changes in carbohydrate intake over a period of 2 years were not associated with either changes in BMI or total body fat or with changes in visceral or subcutaneous adipose tissue [Davis et al., 2009] (LOE IIb).

In 2 children cohorts, one including 41 Australian 8to 9-year-olds, who were followed up after 1 year [Bogaert et al., 2003] (LOE IIb), and the other one including 203 US-American 4-year-olds, who were followed up after 3 years [Klesges et al., 1992] (LOE IIb), there were no associations observed between baseline carbohydrate intake and the subsequent change in BMI z-score or BMI either. In 3 other cohorts, carbohydrate intake at the age of 2 years and above was investigated regarding its prospective effect on body composition. In an Australian cohort of 243 children, an inverse association was observed between the carbohydrate intake from the 2 nd to the 15 th year of live (mean) and the $\mathrm{z}$-score of the triceps skinfold thickness $[\beta(\mathrm{SE})=-0.003(0.001) / \mathrm{g}$ carbohydrates; $\mathrm{p}=$ $0.02]$ as well as the subscapular skinfold thickness $[\beta$ $(\mathrm{SE})=-0.003(0.001) / \mathrm{g}$ carbohydrates; $\mathrm{p}=0.0006]$. For BMI, no association was found [Magarey et al., 2001] (LOE IIb). Higher carbohydrate intake between the 2nd and the 8th year of life (9 measurements) in 70 US-American children was associated with a lower BMI at the age of 8 years [Skinner et al., 2004] (LOE IIb). In a French cohort $(\mathrm{n}=287)$, Rolland-Cachera et al. [1995] (LOE IIb) did not observe an association between carbohydrate intake and subsequent changes in BMI, or the subscapular or triceps skinfold thickness from the 2nd to the 8th year of life. Skinner et al. [2003] (LOE IIb) investigated USAmerican babies from the age of 2 months until the 8th year of life. They found no association between long-term carbohydrate intake (average from up to 15 surveys) and body fat percentage (measured with DEXA) at the age of 8 years either.

For children and adolescents, no targeted intervention studies on the preventive effect of increasing or decreasing the carbohydrate proportion in the diet are available. In the Special Turku Coronary Risk Factor Intervention Project for Children (STRIP) study, babies were randomised at the age of 7 months into an intervention group (target: reduced intake of saturated fat and cholesterol, $\mathrm{n}=540$ ) or a control group (no targeted dietary recommendations, $\mathrm{n}=522$ ). The intervention was carried on until the 14th year of life. The intake of saturated fatty acids and total fat in the course of the study was significantly lower in the intervention group than in the control group, whereas carbohydrate intake was significantly higher. However, differences in carbohydrate intake until the 10th year of life were only 1-2 EN\%; after that they were even smaller [Niinikoski et al., 2007] (LOE Ib). Neither at the 7-year follow-up [Kaitosaari et al., 2003] (LOE Ib) nor at the 14-year follow-up [Niinikoski et al., 2007] (LOE Ib), there was a significant body weight difference between the groups. Furthermore, a pooled analysis of data until the 5th year of life did not show an association between the quantity of fat intake (and the changes of carbohydrate intake accompanied with that) and simultaneous weight increase [Lagström et al., 1999] (LOE IIb).

The available studies regarding children and adolescents mainly suggest that carbohydrate intake or dietary carbohydrate proportion, respectively, is not associated with the risk of obesity. The respective evidence is judged as probable.

\subsection{Mono- and Disaccharides}

\subsubsection{Monosaccharides}

In a 10 -week intervention study including 32 overweight or obese men and women, subjects were asked to cover $25 \%$ of their energy requirements within an ad libitum diet with fructose-sweetened beverages or glucose-sweetened beverages, respectively. Both groups showed comparable body weight gain, but only in the group that had consumed fructose-sweetened beverages, total abdominal fat $(\mathrm{p}<0.05)$ as well as visceral abdominal fat $(\mathrm{p}<0.01)$ increased [Stanhope et al., 2009] (LOE Ib). 
No other prospective cohort studies or well-conducted intervention studies performed for a sufficient period of time are available on the associations between the consumption of monosaccharides and body weight development.

The evidence regarding the relevance of monosaccharides for the risk of obesity is insufficient.

However, there are some cohort and intervention studies on (added) sugar in total as well as specifically on the intake of soft drinks (usually sweetened with HFCS in the USA and with sucrose in Europe), which are presented in the following.

\subsubsection{Disaccharides}

Adults

The relevance of sucrose consumption was investigated in 2 older cohort studies in the USA. In 31,940 nonsmoking female participants of the Nurses' Health Study, higher sucrose intake at baseline was associated with higher body weight gain after 2 years and a lower weight gain after 4 years [Colditz et al., 1990] (LOE IIb). However, a cohort of 465 US-American men and women showed no association between total sucrose intake and a change of BMI in the course of a 12-year follow-up [Parker et al., 1997] (LOE IIb).

In a 10 -week intervention including 49 healthy nonobese women, subjects were asked to replace usual foods by either fat-reduced $(n=17)$ or sugar-reduced $(n=19)$ foods or to maintain their usual diet $(n=13)$, respectively. The intervention resulted in a fat intake reduction by $4 \%$ and sucrose intake reduction by $2.5 \%$, respectively. Body weight change did not differ significantly between the 3 groups [Gatenby et al., 1997] (LOE Ib). There are no other well-conducted intervention studies of sufficiently long duration available.

The evidence regarding the relevance of sucrose or added sugar, respectively, for the risk of obesity in adults is insufficient.

Children and Adolescents

In a collective including 380 German participants of the DONALD study, changing the intake of added sugar between the 2 nd and the 7th year of life was not accompanied by a simultaneous change in BMI or body fat percentage [Buyken et al., 2008] (LOE IIb). A secondary analysis including 543 subjects of the Special Turku Coronary Risk Factor Intervention Project for Children
(STRIP) study did not show an association between sucrose intake and the occurrence of obesity either. However, children with a high average of sucrose intake [highest decile $(n=54)$, mean sucrose intake from the logs from the 1st to the 9th year of life] had a higher BMI between the 1st and the 3rd year of life, but a lower BMI between the 5th and the 9th year of life than children with average $(n=435)$ or low sucrose intake $(n=54)$ [Ruottinen et al., 2008] (LOE IIb). In a group of overweight US-American adolescents of Latin-American origin $(n=85)$, changes in the intake of added or total sugar during a 2-year follow-up were not associated with changes in BMI, total body fat or with changes in the visceral or subcutaneous adipose tissue, respectively [Davis et al., 2009] (LOE IIb). However, a cohort of 519 US-American children showed an inverse association between sucrose intake at the age of 3-4 years and the BMI 4 years later (standardised $\beta=-0.10$ per $g$ sucrose/day; $\mathrm{p}<0.05$ ) [Williams and Strobino, 2008] (LOE IIb).

In a 6-month intervention study including 192 USAmerican families (at least one 7- to 12-year-old child with obesity or at risk of obesity), subjects in the intervention arm were trained to increase their physical activity (2,000 additional steps/day) and to reduce energy intake by $100 \mathrm{kcal} /$ day by replacing typically consumed dietary sugar with non-caloric sweeteners. Compared with the control group, a significantly higher percentage of children in the intervention group were able to either reduce or maintain the BMI $z$-score (67 vs. $53 \%$; $p<0.05$ ) [Rodearmel et al., 2007] (LOE Ib). However, the evaluation of this intervention study regarding the association between sugar intake and body weight development is difficult, as weight reduction might only have been due to the lower energy intake, and because it was a multiple intervention.

The evidence regarding children and adolescents is insufficient.

\subsubsection{Sugar-Sweetened Beverages}

Adults

The meta-analysis performed by Vartanian et al. showed an association between the intake of sugar-sweetened beverages and body weight in adults. The estimated effect size from the 3 cohort studies that have been considered [Bes-Rastrollo et al., 2006; Kvaavik et al., 2005; Schulze et al., 2004b] [r $=0.14$ (95\% CI 0.13; 0.16)] [Vartanian et al., 2007] (LOE IIa) was comparable to the effect size of 5 intervention studies [DiMeglio et al., 2000; Grandjean et al., 2000; Raben et al., 2002; Tordoff et al., 1990; van Wymelbeke et al., 2004] [ $\mathrm{r}=0.15$ (95\% CI 0.05; 
0.24)] [Vartanian et al., 2007] (LOE Ia) and should be rated as medium or low according to the authors' criteria. However, the authors judged the evidence as unambiguous and consistent altogether.

In 2 of the 5 intervention studies considered in the meta-analysis [Grandjean et al., 2000; van Wymelbeke et al., 2004], no effect on body weight was found. However, these studies were repeated experiments, each lasting 1-2 days, on the influence of sugar-sweetened beverages on hydration status and food intake, respectively. The 3 remaining intervention studies (duration of 3-10 weeks) consistently found an increase in body weight after high consumption of sugar-sweetened beverages [DiMeglio et al., 2000; Raben et al., 2002; Tordoff et al., 1990].

Another meta-analysis from 2010 only included intervention studies [Mattes et al., 2010] (LOE Ia). The authors conducted separate analyses for 4 studies on adding mandatory consumption of nutritively sweetened beverages to a person's diet (so-called 'efficacy studies') and for 6 studies on the reduction in sugar-sweetened beverage consumption (so-called 'effectiveness studies'). The latter were almost exclusively studies with children and adolescents (see subparagraph on children and adolescents). The evaluation of adding mandatory consumption of nutritively sweetened beverages also included 1 study that did not investigate the added consumption of sugarsweetened beverages, but the added consumption of a beverage containing milk (7\%) and fruit juice (15\%) [Haub et al., 2005]. The analysis proved a dose-dependent increase in body weight, according to which an added consumption of $250 \mathrm{kcal}$ (about $600 \mathrm{ml}$ ) of nutritively sweetened beverages over a period of 3-12 weeks would result in an about $0.2-\mathrm{kg}$ weight increase [Mattes et al., 2010] (LOE Ia).

Two European and 2 US-American cohort studies were published later than the meta-analyses or were not included into the meta-analyses, respectively: in 288 Dutch workers, increasing the consumption of sugarsweetened beverages by 1 glass/day during a 5 -year follow-up was associated with an increase in body weight of $0.2 \mathrm{~kg} /$ year. However, in the multiple regression model, this association was reduced to $0.12 \mathrm{~kg} /$ year $(\mathrm{p}=0.05)$ [Nooyens et al., 2005] (LOE IIb). In the Cardiovascular Risk in Young Finns Study (2,139 participants), only in women who had increased their consumption of sugarsweetened beverages from childhood and adolescence (3-18 years) to follow-up after 21 years, an association with the risk of obesity was shown [OR $=1.9$ (95\% CI 1.38; 2.62)] [Nissinen et al., 2009] (LOE IIb). A post hoc analysis of the PREMIER Trial, an 18-month randomised con- trolled intervention study on blood pressure reduction including 810 US-American subjects, showed that lowering the consumption of sugar-sweetened beverages by 1 portion/day was accompanied by a simultaneous decrease in body weight by $-0.49 \mathrm{~kg}(95 \% \mathrm{CI}-0.11 ;-0.82$; $\mathrm{p}=0.006)$ after 6 months and by $-0.65 \mathrm{~kg}(95 \% \mathrm{CI}-0.22$; $-1.09 ; \mathrm{p}=0.003$ ) after 18 months, respectively [Chen et al., 2009] (LOE IIb). In the Framingham Heart Study including more than 6,000 middle-aged subjects, the consumption of sugar-sweetened beverages (per portion/ day) led to an increased risk of obesity [OR $=1.31(95 \%$ $\mathrm{CI} 1.02 ; 1.68)]$ or a higher waist circumference $[\mathrm{OR}=1.30$ $(95 \%$ CI $1.09 ; 1.56)]$, respectively, in the course of a 4 -year follow-up [Dhingra et al., 2007] (LOE IIb).

The available cohort and intervention studies regarding adults mainly show that a higher consumption of sugar-sweetened beverages is accompanied by an increased risk of obesity. Therefore, the respective evidence is judged as probable.

\section{Children and Adolescents}

Using cross-sectional, cohort and intervention studies that had been published until 2006, 2 meta-analyses evaluated the evidence in children regarding the association between the consumption of sugar-sweetened beverages and BMI [Forshee et al., 2008] or body weight, respectively [Vartanian et al., 2007]. Forshee et al. [2008] performed a meta-analysis of 8 cohort studies [Berkey et al., 2004; Blum et al., 2005; Ludwig et al., 2001; Mrdjenovic and Levitsky, 2003; Mundt et al., 2006; Newby et al., 2004; Phillips et al., 2004; Striegel-Moore et al., 2006] and 2 intervention studies [Ebbeling et al., 2006; James et al., 2004] regarding changes in BMI. The authors concluded that there was no association between the consumption of sugar-sweetened beverages and BMI [Forshee et al., 2008] (LOE Ia/IIa): increasing the consumption of sugarsweetened beverages by 1 portion/day was accompanied by a non-significant change in BMI of 0.02 (95\% CI $-0.009 ; 0.044)$. However, the financial support of the meta-analysis by the American Beverages Association leaves room for doubt regarding the authors' independence. Furthermore, Forshee et al. [2008] were criticised for their weighting of the studies. Malik et al. [2009] repeated the meta-analysis and excluded studies that adjusted for energy [Blum et al., 2005; Mundt et al., 2006; Newby et al., 2004; Striegel-Moore et al., 2006], as this had been the suspected mechanism of action and had resulted in an underestimation of the effect of sugar-sweetened beverages. According to the re-analysis, the increase in sugar- 
sweetened beverage consumption by 1 portion/day was accompanied by a significant change in BMI of 0.08 (95\% CI 0.03; 0.13) [Malik et al., 2009] (LOE Ia/IIa). Another meta-analysis was based on nearly identical data and concluded that there was a positive association between the consumption of sugar-sweetened beverages and body weight [Vartanian et al., 2007] (LOE Ia/IIa). However, the effects derived from 7 cohort studies [Berkey et al., 2004; Blum et al., 2005; Ludwig et al., 2001; Mrdjenovic and Levitsky, 2003; Newby et al., 2004; Phillips et al., 2004; Striegel-Moore et al., 2006] and 2 intervention studies [Ebbeling et al., 2006; James et al., 2004] are distinctly lower for children and adolescents than for adults: In total, a correlation with body weight of 0.03 (95\% CI 0.02; 0.04 ) was shown, which should be judged as small or close to zero according to the authors' criteria; however, the association regarding the 2 considered intervention studies was greater $[r=0.29(95 \%$ CI $0.29 ; 0.35)]$; this effect size should be judged as medium according to the authors [Vartanian et al., 2007] (LOE Ia).

A new meta-analysis published in 2010 [Mattes et al., 2010] included only intervention studies on the reduction in sugar-sweetened beverage consumption (so-called 'effectiveness studies'). As in previous meta-analyses, the intervention studies by Ebbeling et al. [2006] and James et al. [2004] were included. Furthermore, the authors included 2 additional intervention studies from South America with children and adolescents [Albala et al., 2008; Sichieri et al., 2009] as well as an intervention study with young college students [Munoz, 2006]. The combined analysis of these 5 intervention studies including children, adolescents and young adults did not show any significant effect of lowering the consumption of sugarsweetened beverages on BMI [standardised mean BMI difference -0.037 (95\% CI -0.120; 0.046); $\mathrm{p}=0.6$ ] [Mattes et al., 2010] (LOE Ia). Comparable results were also found without consideration of the young college student study [Munoz, 2006] or with additional consideration of the 2 -year follow-up of James et al. [2007], respectively. However, in a meta-analysis of a subgroup based on the data of 3 studies [Ebbeling et al., 2006; Munoz, 2006; Sichieri et al., 2009], the authors found a consistent effect of the interventions on the BMI of subjects with higher BMI or obesity at baseline [standardised BMI difference 0.35 (95\% CI 0.21;0.49)]. In this subgroup, participants responded to the intervention with a greater weight loss or lower weight gain compared with the control group [Mattes et al., 2010] (LOE Ia).

Since the publication of these meta-analyses, 9 additional cohort studies on the association between the con- sumption of sugar-sweetened beverages and body weight development in children and adolescents have been published until the end of 2009. In a subgroup of participants of the British Avon Longitudinal Study of Parents and Children (ALSPAC), no association was shown between the consumption of sugar-sweetened beverages at the age of 5 $(n=521)$ and 7 years $(n=682)$, respectively, and changes in body fat during the 4-year follow-up [Johnson et al., 2007] (LOE IIb). The analysis of 244 9- to 18 -year-old subjects of the DONALD study revealed a trend regarding an association between the change in the consumption of sugar-sweetened beverages and simultaneous changes in the BMI z-score only for girls. However, increased consumption of fruit juice was associated with a significant increase in BMI z-scores in girls [Libuda et al., 2008] (LOE IIb). Project EAT including 2,294 adolescents showed no association between the consumption of sugar-sweetened beverages and BMI changes during a 5-year follow-up. According to the authors, the direct association with low-energy soft drinks $(p=0.002)$ was due to a targeted modification of eating behaviour for weight reduction [Vanselow et al., 2009] (LOE IIb). In 268 US-American adolescents, no associations between the consumption of sugar-sweetened beverages at baseline and changes in BMI during an 18-month follow-up were shown [Laurson et al., 2008] (LOE IIb). Dubois et al. [2007] (LOE IIb) found a more than 2-fold higher risk of obesity at the age of 4.5 years in 1,944 Canadian children who consumed sugar-sweetened beverages between meals on a regular basis already at the age of $2.5,3.5$ and 4.5 years. In a study including 49 US-American children, there was an associaton between higher consumption of sugar-sweetened beverages between the $3 \mathrm{rd}$ and the 6 th year of life and the simultaneous change in waist circumference, but not in BMI z-score; in this study, children with familial increased risk of obesity consumed significantly more sugar-sweetened beverages already at the age of 3 years [Kral et al., 2008] (LOE IIb). In 365 Afro-American preschool children, the intake of sugar-sweetened beverages at baseline was associated with an increased risk of developing overweight in the 2-year follow-up [Lim et al., 2009] (LOE IIb). In another study including 166 US-American girls, the consumption of sugar-sweetened beverages at the age of 5 years was predictive for a higher body fat percentage, greater waist circumference and weight gain by the age of 15 years [Fiorito et al., 2009] (LOE IIb). In 268 subjects of an Australian cohort, there was an association between the consumption of sugar-sweetened beverages during childhood and subsequent weight gain in the 5-year follow-up [Tam et al., 2006] (LOE IIb). 
From 2 of the 4 available meta-analyses the conclusion is drawn that increased consumption of sugar-sweetened beverages in children and adolescents is associated with a higher risk of obesity. In contrast, another meta-analysis judges the effect as almost zero. The cohort studies published since then verify this risk-increasing effect only in part. The most recent meta-analysis concludes that the risk-increasing effect is limited to individuals with initially already increased BMI or existing overweight, respectively. The overall evidence regarding an increased risk of obesity due to higher consumption of sugar-sweetened beverages in children and adolescents is therefore only judged as possible.

The evidence regarding an increased risk of obesity especially in children and adolescents with initially already increased BMI or already existing overweight is also judged as possible.

The judgement of the evidence regarding sugar-sweetened beverages altogether is impeded due to a possible publication bias. Vartanian et al. [2007] for example showed that the majority of studies sponsored by the sugar industry do not find an association with the risk of obesity. On the other hand, since the public health sector expects a risk-increasing association, this could lead to preferred publication of confirming studies [Gibson, 2008]. The judgement of the evidence regarding the role of sugar-sweetened beverages in the prevention of obesity therefore is particularly based on the available intervention studies.

\subsection{Polysaccharides}

No cohort or intervention studies were identified that were relevant for the present question.

\subsubsection{Refined Grain Products}

Adults

In 2 evaluations of observational data of the Danish Diet, Cancer and Health Study including about 45,000 women and men, an increased consumption of refined bread was associated with an increase in waist circumference in women only $[\beta=0.42(95 \% \mathrm{CI} 0.11 ; 0.73) \mathrm{cm} /$ quintile rise of consumption; $p<0.05$ ] [Halkjaer et al., 2004] (LOE IIb). Likewise, a higher carbohydrate intake due to refined grain products and potatoes was associated with an increase in waist circumference $[\beta=0.48$ ( $95 \%$ CI 0.18 ; $0.78) \mathrm{cm} / \mathrm{MJ}$ carbohydrates from these sources; $\mathrm{p}=0.002$ ] [Halkjaer et al., 2006] (LOE IIb). Regarding men, no asso- ciations were found [Halkjaer et al., 2004, 2006] (both LOE IIb). In the Nurses' Health Study, modification of the intake of refined grain products over a period of 12 years was associated with a change in body weight and the risk of developing obesity. While women with the lowest increase in the intake of refined grain products (quintile 1) had an average weight gain of $4.3 \mathrm{~kg}$, women with the highest increase in intake (quintile 5) showed a weight gain of 4.7 $\mathrm{kg}$ and had a higher risk of developing obesity [adjusted $\mathrm{OR}=1.18$ (95\% CI 1.08; 1.28); $\mathrm{p}=0.0001$ ] [Liu et al., 2003] (LOE IIb). However, in the Physicians' Health Study including 17,881 men, a higher intake of refined breakfast cereals at baseline was associated with lower weight gain after 8 years [consumption of at least 1 portion/day vs. rare consumption: 0.94 (0.16) kg vs. $1.46(0.05) \mathrm{kg} ; \mathrm{p}=0.005$ ]. However, after 13 years, no comparable trend could be shown ( $\mathrm{p}=0.3$ ) [Bazzano et al., 2005] (LOE IIb).

The evidence regarding the relevance of refined grain products for the risk of obesity is judged as insufficient.

\section{Children and Adolescents}

No cohort or intervention studies were identified that were relevant for the present question.

\subsubsection{Dietary Fibre}

Cohort studies on the consumption of dietary fibre investigated both the intake in g/day and the dietary fibre density. Both approaches are considered together in the following; for details on the influencing variables, see the tables for each health outcome (http://www.dge.de/leitlinie; German only).

\section{Adults}

As part of the DIOGenes Project, in the EPIC Study including 89,432 subjects a significant inverse association was found between dietary fibre intake and subsequent change in body weight or waist circumference, respectively, during a mean follow-up of 6.5 years. A dietary fibre intake increase by $10 \mathrm{~g}$ was associated with an average body weight decrease of $-39 \mathrm{~g} /$ year $(95 \%$ CI $-71 ;-7)$ and an average waist circumference decrease of -0.08 $\mathrm{cm} /$ year (95\% CI -11; -0.05) [Du et al., 2010] (LOE IIb). A study including 288 Dutch workers found a lowering effect of an increase in dietary fibre density on body weight and waist circumference, respectively, during a 5-year follow-up ( $\beta=-0.31 \mathrm{~kg}$ per $\mathrm{g} / \mathrm{MJ}$ increase in 5 years, $\mathrm{p}=$ 0.01 ; and $\beta=-0.32 \mathrm{~cm}$ per $\mathrm{g} / \mathrm{MJ}$ increase in 5 years, $\mathrm{p}<$ 0.01 , respectively). However, these associations were not significant anymore after adjusting for other lifestyle fac- 
tors $(\beta=-0.17, p=0.10$; or $\beta=-0.08, p=0.6)$ [Nooyens et al., 2005] (LOE IIb). Karnehed et al. [2006] (LOE IIb) investigated 952 young men from the Swedish Twin Registry and found that low dietary fibre intake compared with high intake was associated with an increase of 0.45 BMI units $(95 \%$ CI 0.15 ; 0.76) during a follow-up of 4 years. However, in 1,762 participants of the Danish MONICA arm, no association between baseline dietary fibre intake and subsequent body weight development over 5 years could be detected [Iqbal et al., 2006] (LOE IIb). Both in the Nurses' Health Study and in the Health Professionals Follow-Up Study, an increase in dietary fibre intake over 12 and 8 years, respectively, was associated with a significant reduction in body weight [Liu et al., 2003; Koh-Banerjee et al., 2004] (both LOE IIb). The risk of overweight and obesity, respectively, was significantly lower in women in the highest quintile of dietary fibre intake than in women in the lowest quintile of dietary fibre intake [adjusted OR $=0.51(95 \%$ CI $0.39 ; 0.67)$, $\mathrm{p}$ for trend $<0.0001$; and $\mathrm{OR}=0.66(95 \% \mathrm{CI} 0.58 ; 0.74)$, $\mathrm{p}$ for trend $<0.0001$ )] [Liu et al., 2003] (LOE IIb). In an analysis of the Nurses' Health Study from 1990, increased dietary fibre intake at baseline was accompanied by weight reduction after 2 years; however, increased dietary fibre intake at baseline was also associated with increased weight gain after 4 years [Colditz et al., 1990] (LOE IIb). In young US-American participants of the CARDIA study, again a protective effect of high dietary fibre intake was found: men and women with the highest dietary fibre intake at baseline had significantly lower body weight 10 years later than subjects with the lowest baseline dietary fibre intake (Caucasian women and men: quintile 1: 78.7 $\mathrm{kg}$ vs. quintile 5: $75.0 \mathrm{~kg}, \mathrm{p}<0.001$; Afro-Americans: quintile $1: 83.5 \mathrm{~kg}$ vs. quintile $5: 79.9 \mathrm{~kg}, \mathrm{p}=0.001)$. Only in Caucasian subjects, an association with a lower waistto-hip ratio was seen $(\mathrm{p}=0.004)$ [Ludwig et al., 1999] (LOE IIb). In 116 US college students, a lower dietary fibre intake at baseline, at least in female subjects, was associated with an increase in BMI after 1 year $(\mathrm{p}=0.01)$ [Adams and Rini, 2007] (LOE IIb). Dietary fibre intake and changes in body composition were also associated in a cohort of 252 US-American women during a follow-up of 20 months. A $1 \mathrm{~g} / 1,000 \mathrm{kcal}$ higher dietary fibre intake was accompanied by a decrease in body weight of $0.25 \mathrm{~kg}$ $(\mathrm{p}=0.0061)$ and a reduction in body fat percentage of $0.25 \%(\mathrm{p}=0.0052)$ [Tucker and Thomas, 2009] (LOE IIb). Intervention studies on the preventive effect of increased dietary fibre intake are not available. In a 1-year pilot study on the prevention of obesity including 40 young women with familial predisposition to obesity, in which modification of dietary fibre intake was not part of the planned intervention, an increase in dietary fibre density was inversely associated with the achieved weight change $[\beta(\mathrm{SE})=-0.21(0.06) \mathrm{kg}$ body weight/increase in dietary fibre intake in $\mathrm{g} / 1,000 \mathrm{kcal} ; \mathrm{p}=0.002$ ] [Eiben and Lissner, 2006] (LOE Ib). In addition, in the Women's Health Initiative Dietary Modification Trial (WHI) including about 49,000 post-menopausal US-American women, an increase in dietary fibre intake was associated in the course of a 7.5-year follow-up with a trend towards weight reduction according to the multivariate secondary analysis [Howard et al., 2006] (LOE IIb).

The majority of the cohort studies indicate that increased dietary fibre intake is associated with a reduced risk of obesity. Therefore, the evidence regarding this association is judged as probable.

\section{Children and Adolescents}

In the Danish participants of the European Youth Heart Study ( $\mathrm{n}=398,8$ - to 10-year-olds), only for boys with normal weight an inverse association was shown between changed dietary fibre intake and simultaneous change in BMI z-score ( $p$ for interaction $=0.02$ ): an increase by $10 \mathrm{~g} /$ day was associated with a decrease by 0.15 z-scores in the course of the 3-year study period [Iqbal Kring and Heitmann, 2008] (LOE IIb). The DONALD study showed no association during adolescence (from the onset of puberty to 4 years later) between a change of dietary fibre intake and the accompanied development of BMI and body fat [Cheng et al., 2009] (LOE IIb). However, in children who consumed a maximum of 5 meals per day during infancy, an increase in dietary fibre intake between the 2 nd and the 7 th year of life was accompanied by a reduction in body fat $[-0.26 \%$ body fat/increase in dietary fibre intake by $1 \mathrm{SD}$ (ca. $4 \mathrm{~g} /$ day); $\mathrm{p}=0.005$ ]; in the control group with a higher frequency of meals, no such association was observed $(0.07 \%$ body fat/increase in dietary fibre intake by $1 \mathrm{SD} ; \mathrm{p}=0.3)$ [Buyken et al., 2008] (LOE IIb). Berkey et al. [2000] (LOE IIb) did not find an association between changes in dietary fibre intake and simultaneous changes in body weight in almost 11,000 9- to 14-year-old US-American children during a 1-year follow-up. Similarly, Williams and Strobino [2008] (LOE IIb) did not find an association with the BMI 4 years later in an investigation of 519 3- to 4-year-old US-American children. However, in a group of overweight adolescents of Latin-American origin $(n=85)$, subjects who reduced their dietary fibre intake (by an average of $3 \mathrm{~g} / 1,000$ $\mathrm{kcal}$ ) in the 2-year follow-up significantly gained visceral 
fat compared with those with increased dietary fibre intake (+21 vs. $-4 \% ; \mathrm{p}=0.02$ ) [Davis et al., 2009] (LOE IIb).

Intervention studies on the preventive effect of increased dietary fibre intake in children and adolescents are not available.

Regarding children and adolescents, the cohort studies available show with possible evidence that there is no association between dietary fibre intake and the risk of obesity. The relevance of dietary fibre intake in children and adolescents might depend on the initial body weight, but the respective evidence is insufficient.

\subsubsection{Whole-Grain Products}

Adults

In 2 analyses of the cohort study Danish Diet, Cancer and Health Study including about 45,000 women and men, no associations were shown between the consumption of whole-grain bread or carbohydrate intake from whole-grain products at baseline and change in body weight during a follow-up of 5-6 years [Halkjaer et al., 2004, 2006] (both LOE IIb). In the Nurses' Health Study ( $\mathrm{n}=74,091)$, women with the lowest increase in wholegrain intake (quintile 1) during a 12-year follow-up gained an average of $4.5 \mathrm{~kg}$ weight, while women with the highest increase in whole-grain intake (quintile 5) gained 4.1 $\mathrm{kg}$ weight and had a lower risk of developing obesity [adjusted OR $=0.81$ (95\% CI 0.73; 0.91); $\mathrm{p}=0.0002$ ] [Liu et al., 2003] (LOE IIb). In the Health Professionals Follow-Up Study ( $\mathrm{n}=27,082)$, an increase in whole-grain product intake in men during an 8-year follow-up was also associated with a lower weight gain [quintile $1:+1.24(0.23) \mathrm{kg}$ vs. quintile $5:+0.75(0.22) \mathrm{kg} ; \mathrm{p}<0.0001$ ] [Koh-Banerjee et al., 2004] (LOE IIb). Bazzano et al. [2005] (LOE IIb) showed in the Physicians' Health Study (17,881 men) that the adjusted body weight gain after 8 years was significantly higher in men who had consumed whole-grain breakfast cereals only rarely at baseline compared to those men who had consumed at least 1 portion/day at baseline [1.55 (0.55) kg vs. $1.13(0.11) \mathrm{kg} ; \mathrm{p}=0.003]$. The follow-up examination after 13 years showed a similar tendency $(\mathrm{p}=0.08)$.

There are no intervention studies on the primary prevention of obesity through whole-grain products.

The few existing cohort studies regarding adults mainly suggest that increased whole-grain product intake is accompanied by a reduced risk of obesity. The overall evidence is judged as possible.

Evidence-Based Guideline of the German Nutrition Society

\section{Children}

The DONALD study showed no association during adolescence (from the onset of puberty to 4 years later) between changes in whole-grain product intake and the accompanied BMI development and body fat, respectively [Cheng et al., 2009] (LOE IIb).

There are no intervention studies on the primary prevention of obesity in children and adolescents through whole-grain products.

For children and adolescents, the evidence regarding the role of whole-grain product intake in the development of obesity is insufficient.

\subsection{Glycaemic Index and Glycaemic Load}

\section{Adults}

In 191 female participants of the Danish MONICA arm, an association was shown between GI at baseline and body composition 6 years later. Higher baseline GI was associated with a moderate subsequent increase in body weight (logarithmic, $\mathrm{p}<0.05$ ) and body fat percentage $[\beta=0.09 \%$ per GI unit (GI reference: white bread) (95\% CI 0.004; 0.17), $\mathrm{p}<0.05]$. For diet GL, there were no prospective associations found in women; and in the 185 male subjects, neither GI nor GL were associated with body composition development [Hare-Bruun et al., 2006] (LOE IIb). As part of the DIOGenes Project in the EPIC Study including 89,432 subjects, no significant association was found between GI and GL, respectively, and change in body weight over an average of 6.5 years. Higher baseline GI, however, resulted in greater increase in waist circumference $[0.19 \mathrm{~cm}$ per year $(95 \%$ CI $0.11 ; 0.27)$ per GI difference of 10 units]; but this association was heterogeneous between the different European study populations combined in this study [Du et al., 2009] (LOE IIb). In a cohort of 641 healthy US-American women and men, who were examined every 3 months over 1 year, an increase in diet GI by 5 units was associated with a BMI increase by 0.04 units $(95 \%$ CI $0.01 ; 0.07)(p=0.02)$. No associations with diet GL have been observed [Ma et al., 2005] (LOE IIb).

In a $2 \times 12$-week (crossover) intervention study including 19 overweight and obese women, no influence of GI on changes in body weight, waist circumference or body fat percentage was observed. The aim of the study was not weight reduction; the intervention only comprised the replacement of 4 main sources of carbohydrates within the ad libitum consumption of every day

Ann Nutr Metab 2012;60(suppl 1):1-58 
diet by foods with either high GI or low GI. According to the 4-day-weighing records from the end of each period, a GI difference of 8.4 units was achieved [Aston et al., 2008] (LOE Ib).

The few exisisting studies suggest possible evidence in women regarding the association of an increased GI with increased risk of obesity, while in men the evidence regarding such an association is insufficient.

Regarding GL, the 3 cohort studies consistently suggest that there is no association with the risk of obesity. The respective evidence is judged as possible.

\section{Children and Adolescents}

In 2 prospective analyses of the DONALD study, no association between changes in GI or GL and simultaneous development of BMI and body fat percentage, respectively, was found for either childhood (2-7 years) or adolescence (from the onset of puberty to 4 years later), [Buyken et al., 2008; Cheng et al., 2009] (LOE IIb). However, different relevances were indicated regarding GI increase and simultaneous development of body fat percentage or BMI-SDS, respectively, depending on whether the adolescents already had overweight at the onset of puberty or not $(p=0.03$ or 0.08 for interaction GI $\times$ overweight at baseline $\times$ change in body composition) [Cheng et al., 2009] (LOE IIb). In a group of overweight USAmerican adolescents of Latin-American origin $(n=85)$, changes in GI or GL during the 2-year follow-up were not associated with changes in BMI, total body fat or with changes in the visceral or subcutaneous adipose tissue, respectively [Davis et al., 2009] (LOE IIb).

There are no intervention studies available on the primary prevention of obesity.

Regarding children and adolescents, the evidence on the role of GI and GL, respectively, concerning the development of obesity is insufficient.

\subsection{Need for Research regarding Obesity}

For children and adolescents, in particular, the data basis concerning the relevance of carbohydrates in the prevention of obesity is still insufficient. Especially studies on the relevance of whole-grain product intake as well as on GI and GL are still required. A number of cohort studies are available on sugar-sweetened beverages. However, further intervention studies would be desirable.
For the identification of possible vulnerable groups, separate approaches are important, especially for children and adolescents with initially already increased BMI or existing obesity. Besides, targeted intervention studies regarding all aspects of carbohydrate intake seem to be feasible only for these risk groups.

Considering the respective insufficient evidence, current intervention and cohort studies on the relevance of sucrose, total sugar and added sugar regarding children, adolescents and adults would be interesting.

Targeted intervention studies on reducing the risk of obesity by means of increased consumption of wholegrain products would be desirable so as to draw firmer conclusions regarding the evidence for a protective effect of whole-grain products in adults, which is presently judged as 'possible'.

\section{Quantity and Quality of Carbohydrate Intake and Primary Prevention of Type 2 Diabetes Mellitus}

\subsection{Proportion of Carbohydrates}

In prospective cohort studies, the role of the proportion of carbohydrates in the diet or the quantity of carbohydrates, respectively, was investigated regarding the occurrence of type 2 diabetes mellitus. These studies greatly vary in population size and study duration, but also in particular in the investigated exposure variable and its multivariate modelling. In some studies, the absolute quantity of carbohydrates in the diet was investigated, while in others the relative proportion of carbohydrates in the diet was evaluated either as percentage of total energy intake or by means of energy adjustment with the residual method.

The absolute quantity of carbohydrate intake (not energy-adjusted) was investigated in 3 cohort studies which showed varying results. In one part of the Seven Countries Study, the Zutphen Study, including 176 men, a positive association was observed between absolute carbohydrate intake and the risk of diabetes over a 3-year period (OR for extreme tertiles $=2.97 ;$ 95\% CI 1.30; 6.79) [Feskens et al., 1991] (LOE IIb). In an Australian cohort study including 2,123 men and women, no association between carbohydrate intake and the risk of diabetes was observed over a period of 10 years [multivariate-adjusted relative risk $(\mathrm{RR})$ per $200 \mathrm{~g} / \mathrm{day}=1.14 ; 95 \% \mathrm{CI} 0.43 ; 3.00, \mathrm{p}=0.79]$ [Barclay et al., 2007] (LOE IIb). In the EPIC-Potsdam Study (9,702 men, 15,365 women), absolute carbohydrate intake was not significantly associated with the risk of 
diabetes, if adjustment for important risk factors was done (RR extreme quintiles: men $=0.67 ; 95 \%$ CI 0.41 ; 1.08 ; women $=0.81 ; 95 \%$ CI $0.46 ; 1.44$ ) [Schulze et al., 2008] (LOE IIb).

Due to the strongly varying results and the low number of cohort studies available so far, the evidence regarding an association between the absolute intake of carbohydrates and the risk of type 2 diabetes mellitus is considered to be insufficient.

Several studies investigated the proportion of carbohydrate intake of total energy intake (EN\%) or as energyadjusted intake quantity in grams per day. The majority of these studies suggested that the relative carbohydrate proportion of the diet does not influence the risk of diabetes. In a Swedish cohort $(n=1,462)$, the proportion of carbohydrates of total energy intake did not differ between women who developed type 2 diabetes mellitus and women without diabetes after an observation period of 12 years [Lundgren et al., 1989] (LOE IIb). In the Nurses' Health Study ( $=84,360)$, the risk of women with higher carbohydrate intake (energy-adjusted) to develop diabetes was not significantly different from the risk of women with lower intake over 6 years of followup (RR comparing extreme quintiles of carbohydrate intake in women with BMI <29: $1.31, \mathrm{p}=0.42$; in women with BMI $\geq 29: 1.13, \mathrm{p}=0.39$ ) [Colditz et al., 1992] (LOE IIb). Salmeron et al. [1997b] (LOE IIb) investigated a later follow-up period in the same cohort (1986-1992, 65,173 women). The RR comparing extreme quintiles of the energy-adjusted carbohydrate intake was 1.04 (95\% CI 0.83; 1.30). In the San Luis Valley Diabetes Study, subjects who developed diabetes during a 1- to 3-year follow-up period $(\mathrm{n}=20)$ had a lower mean relative carbohydrate intake of $40.5 \mathrm{EN} \%$ compared with subjects who still had impaired glucose tolerance ( $\mathrm{n}=43,43.5 \mathrm{EN} \%$ carbohydrates) and subjects who reverted to normal glucose tolerance ( $\mathrm{n}=60,45.1$ EN\% carbohydrates) $(\mathrm{p}=$ 0.08 ), with adjustment for age, gender and ethnic origin [Marshall et al., 1994] (LOE IIb). In the Seven Countries Study, including 338 men, the proportion of carbohydrates of total energy intake at baseline and its change during the 20-year follow-up was not significantly associated with the risk of diabetes [Feskens et al., 1995] (LOE IIb). Energy-adjusted carbohydrate intake in the prospective Health Professionals Follow-Up Study (42,759 US-American male health-care professionals) was not associated with the risk of type 2 diabetes mellitus $(\mathrm{RR}$ comparing extreme quintiles $=0.85 ; 95 \% \mathrm{CI}$
0.62; 1.15) [Salmeron et al., 1997a] (LOE IIb). In the Iowa Women's Health Study including 35,988 women (6 years of follow-up), there was no association between energyadjusted carbohydrate intake and the risk of diabetes either (RR comparing extreme quintiles $=0.93 ; 95 \% \mathrm{CI}$ 0.76; 1.13) [Meyer et al., 2000] (LOE IIb). In the EPICNorfolk Study, no association between the proportion of carbohydrates of total energy intake and the risk of diabetes during 3-7 years of follow-up could be identified (OR 0.99 or 1.01) in either 9,611 men or 11,861 women [Harding et al., 2004] (LOE IIb). Likewise in the EPICPotsdam Study, the proportion of carbohydrates in total energy intake was not associated with the risk of diabetes $[\mathrm{RR}$ comparing extreme quintiles $=0.91(95 \% \mathrm{Cl} 0.66$; 1.26) among men and 0.89 (95\% CI $0.41 ; 1.08)$ among women] [Schulze et al., 2008] (LOE IIb). In contrast to these studies, a higher energy-adjusted carbohydrate intake in the Shanghai Women's Health Study $(64,227$ women, 4.6 years of follow-up) was associated with an increased risk of diabetes (RR comparing extreme quintiles $=1.28$ (95\% CI 1.09; 1.50) [Villegas et al., 2007] (LOE IIb). In Pima Indians, carbohydrate intake was not significantly associated with the risk of type 2 diabetes mellitus in a model adjusting for total energy intake (RR comparing extreme quartiles $=2.2 ; 95 \%$ CI $0.3 ; 16.7$ ) [Williams et al., 2001] (LOE IIb).

In the Women's Health Initiative Randomized Controlled Dietary Modification Trial (WHI) including 48,835 post-menopausal women, subjects in the intervention arm were motivated to reduce their fat intake. The intervention resulted in an increase in carbohydrate percentage of the diet $(52.7 \%$ vs. $44.7 \%$ of total energy intake after year $1 ; 54.1 \%$ vs. $45.9 \%$ after year 6 ) to a large extent at the expense of the fat percentage $(24.2 \%$ vs. $35.0 \%$ and $28.6 \%$ vs. $36.9 \%$, respectively). Women in the intervention group lost slightly more weight during the first year than women in the control group (mean body weight 74.0 vs. $75.9 \mathrm{~kg}$ ); after 6 years there was only a minor difference (75.6 vs. $76.2 \mathrm{~kg}$ ). Between intervention and control group there was no difference in the incidence of type 2 diabetes mellitus during 9 years of follow-up ( $\mathrm{RR}=0.96$; 95\% CI 0.90; 1.03) [Tinker et al., 2008] (LOE Ib).

It can be concluded from the available data that primary prevention of type 2 diabetes mellitus through increasing the carbohydrate proportion is not possible. The majority of the cohort studies as well as a large randomised intervention study result in convincing evidence that there is no association. 
In addition, 4 prospective cohort studies also investigated nutrient substitutions with clearly heterogeneous results. In the Nurses' Health Study II $(91,249$ women, 8-year follow-up), no significant relation between carbohydrate intake and the risk of diabetes was observed $(\mathrm{RR}$ comparing extreme quintiles $=0.89 ; 95 \%$ CI $0.60 ; 1.33)$; due to adjustment for dietary fats this result can be interpreted as substitution of proteins with carbohydrates [Schulze et al., 2004a] (LOE IIb). However, modelling of specific substitution models in the EPIC-Potsdam Study showed that isocaloric exchange of proteins for carbohydrates is associated with reduced risk $(\mathrm{RR}$ per $5 \%$ energy $=0.77 ; 95 \%$ CI $0.64 ; 0.91)$. In the same study, there was no association when exchanging total dietary fat for carbohydrates [Schulze et al., 2008] (LOE IIb). In contrast, in the Nurses' Health Study (85,059 women) Halton et al. [2008] (LOE IIb) observed a higher risk of diabetes with higher carbohydrate intake during a follow-up period of 20 years (RR comparing extreme deciles $=1.26 ; 95 \% \mathrm{CI} 1.07 ; 1.49$, $\mathrm{p}$ trend $=$ $0.003)$. In this analysis, adjustment for protein and total energy intake was performed, so that higher carbohydrate intake has to be interpreted as isocaloric substitution of total fat. In the Nurses' Health Study, isoenergetic substitution of MUFA as well as SFA with carbohydrates did not result in an association with the risk of type 2 diabetes mellitus, while the substitution of PUFA with carbohydrates was associated with an increased risk (RR per $5 \mathrm{EN} \%=1.58 ; 95 \%$ CI $1.31 ; 1.90)$ and the substitution of trans-fatty acids with carbohydrates was associated with a lower risk (RR per $2 \mathrm{EN} \%=0.72 ; 95 \%$ CI 0.60; 0.87) [Salmeron et al., 2001] (LOE IIb).

Due to the strongly varying results and the low number of cohort studies available so far, the evidence regarding an association between increasing the intake of carbohydrates at the expense of other specific energy-yielding nutrients and the risk of type 2 diabetes mellitus is considered to be insufficient.

\subsection{Mono- and Disaccharides}

Total Amount of Mono- and Disaccharides

Some studies investigated the total intake of monoand disaccharides regarding possible associations with the risk of diabetes. In Australian adults participating in the Melbourne Collaborative Cohort Study ( $\mathrm{n}=36,787$ ), total intake of mono- and disaccharides was inversely associated with the risk of diabetes (OR per 100 g/day: 0.61;
95\% CI 0.47; 0.79). This association was still significant after further adjustment for BMI and waist-to-hip ratio [Hodge et al., 2004] (LOE IIb). In the Women's Health Study, no significant relation between the intake of monoand disaccharides and the risk of diabetes was observed (RR comparing extreme quintiles $=0.86$; 95\% CI 0.69; 1.06 , $p$ trend $=0.17$ ) [Janket et al., 2003] (LOE IIb). In the Finnish Mobile Clinic Health Examination Survey (4,304 men and women) Montonen et al. [2007] (LOE IIb) observed no significant relation between the total intake of mono- and disaccharides and the incidence of diabetes ( $\mathrm{RR}$ comparing extreme quartiles $=1.42 ; 95 \%$ CI 0.90 ; 2.24).

Due to the varying results, the evidence regarding an association between the total intake of mono- and disaccharides and the risk of type 2 diabetes mellitus is judged as insufficient.

Fructose and Glucose

In the Iowa Women's Health Study, the intake of fructose $(\mathrm{RR}=1.27 ; 95 \% \mathrm{CI} 1.06 ; 1.54)$ and glucose $(\mathrm{RR}=1.30$; $95 \%$ CI 1.08 ; 1.57) was positively associated with the risk of diabetes [Meyer et al., 2000] (LOE IIb). In the Finnish Mobile Clinic Health Examination Survey, the intake of glucose $(1.68 ; 1.06-2.65)$ or fructose $(1.62 ; 1.01-2.59)$ and the sum of the intake of both carbohydrates $(1.57 ; 1.00$ 2.48) was positively associated with the risk of diabetes [Montonen et al., 2007] (LOE IIb). In contrast, in the Women's Health Study there was no significant association between fructose and glucose intake and the risk of diabetes [Janket et al., 2003] (LOE IIb). In the EPIC-Potsdam Study, no association was observed between fructose or glucose intake and the risk of diabetes [Schulze et al., 2008] (LOE IIb).

The evidence regarding an association between the intake of glucose and fructose and the risk of diabetes is insufficient.

\section{Sucrose}

In the San Antonio Heart Study, sucrose intake at baseline examination did not differ significantly between subjects who got diabetes during follow-up and subjects who stayed healthy [Monterrosa et al., 1995] (LOE IIb). In the Nurses' Health Study ( $\mathrm{n}=84,360)$, the risk of developing diabetes during 6 years of follow-up did not differ between women with higher and women with lower sucrose intake (RR comparing extreme quintiles of sucrose intake in women with: $\mathrm{BMI}<29: 1.16, \mathrm{p}=0.76$; for women with BMI 
$\geq 29: 0.90, p=0.20)$ [Colditz et al., 1992] (LOE IIb). In the Women's Health Study there was no significant association between sucrose intake and the risk of diabetes either [Janket et al., 2003] (LOE IIb). In the EPIC-Potsdam Study, no significant association between sucrose intake and the risk of diabetes could be found in either men (RR comparing extreme quintiles $=0.72 ; 95 \%$ CI $0.50 ; 1.04)$ or women $(1.13$; 0.74-1.74) [Schulze et al., 2008] (LOE IIb). In the Finnish Mobile Clinic Health Examination Survey, Montonen et al. [2007] (LOE IIb) did not observe an association between sucrose intake and the risk of diabetes $(\mathrm{RR}=1.22 ; 95 \% \mathrm{CI}$ $0.77 ; 1.92)$. In the Iowa Women's Health Study including 35,988 women ( 6 years of follow-up), there was an inverse association between energy-adjusted sucrose intake and the risk of diabetes (RR comparing extreme quintiles = 0.81; 95\% CI 0.67; 0.99) [Meyer et al., 2000] (LOE IIb).

Due to the inconsistency of the results, the evidence regarding a lack of an association between sucrose intake and the risk of type 2 diabetes mellitus is judged as probable.

\section{Lactose}

Regarding the intake of lactose, no relation with the risk of diabetes was observed in the Iowa Women's Health Study $(\mathrm{RR}=0.94 ; 95 \%$ CI 0.77; 1.14) [Meyer et al., 2000] (LOE IIb). There was no significant association with the risk of diabetes in the Women's Health Study either [Janket et al., 2003] (LOE IIb). In addition, lactose did not have any influence on the risk of diabetes in the Finnish Mobile Clinic Health Examination Survey [Montonen et al., 2007] (LOE IIb).

In spite of homogeneous study results, the evidence regarding a lack of an association between lactose intake and the risk of diabetes is judged as possible due to the low number of studies.

\section{Sugar-Sweetened Beverages}

In addition to the intake of mono- and disaccharides, some studies have investigated the consumption of sugarsweetened beverages. While in the USA these beverages are usually sweetened with HFCS which mostly contains $55 \%$ fructose and $45 \%$ glucose, in Europe sucrose is normally used. In the Nurses' Health Study II including 91,249 women, a positive association was shown between the consumption of sugar-sweetened beverages and the risk of diabetes over a period of 8 years. The risk of diabetes increased by $83 \%$ (95\% CI 1.42; 2.36) in women who consumed these beverages on a daily basis compared to women who consumed these beverages only rarely $(<1$ time/month). This positive relation was only partly reduced due to adjustment for the participants' BMI [Schulze et al., 2004b] (LOE IIb). Positive associations were also observed in the Finnish Mobile Clinic Health Examination Survey (RR comparing extreme quartiles $=1.60 ; 95 \%$ CI 0.93; 2.76; p trend $=0.01$ ) [Montonen et al., 2007] (LOE IIb) and in the Black Women's Health Study (RR $\geq 2$ portions/day compared with $<1$ portion/month $=1.24 ; 95 \%$ CI 1.06; 1.45) [Palmer et al., 2008] (LOE IIb). In contrast, there was no association between the consumption of sugar-sweetened beverages and the risk of diabetes in the Atherosclerosis Risk in Communities Study [Paynter et al., 2006] (LOE IIb). In a meta-analysis from 2010, additionally to these studies the results of 4 more current prospective cohort studies were included, so that the evaluation was based on the data of 310,819 participants $(15,043$ cases) [Malik et al., 2010] (LOE IIa). Comparing highest and lowest intake (between $<1$ portion/month and $\geq 1-2$ portions/day) revealed a significantly increased risk of diabetes $(\mathrm{RR}=1.26,95 \% \mathrm{CI} 1.12 ; 1.41)$. The study results were largely consistent: 8 out of 9 showed a positive association. There were no signs of publication bias, as the degree of association was independent of the study size.

The majority of prospective cohort studies and 1 metaanalysis thereof indicate an increased risk of type 2 diabetes mellitus due to regular consumption of sugar-sweetened beverages. The evidence regarding this association is judged as probable.

\subsection{Polysaccharides}

Some studies investigated if the intake of polysaccharides was associated with the risk of diabetes. In Australian adults participating in the Melbourne Collaborative Cohort Study $(\mathrm{n}=36,787)$, the intake of starch was positively associated with the risk of diabetes (OR per $100 \mathrm{~g} /$ day $=1.47 ; 95 \%$ CI 1.06; 2.05). This association was still significant after further adjustment for BMI and waistto-hip ratio [Hodge et al., 2004] (LOE IIb). In a study on Pima Indians, the intake of complex carbohydrates was not associated with the risk of diabetes (RR comparing extreme quartiles $=1.2$; 95\% CI 0.2; 6.9) [Williams et al., 2001] (LOE IIb). However, starch and dietary fibre were analysed together. Therefore, the role of starch alone remains unclear. Studies on the intake of fibre-poor refined grain products also provide some information. Fung et al. [2002] (LOE IIb) could not identify an association between the intake of refined grain products and the risk of 
diabetes over a 12-year follow-up period (1986-1998) in the Health Professionals Follow-Up Study (RR comparing extreme quintiles $=1.08 ; 95 \%$ CI $0.87 ; 1.33)$. However, van Dam et al. [2002] (LOE IIb) reported a significant positive association in the same cohort and identical follow-up period with refined grain products (RR comparing extreme quintiles $=1.32 ; 95 \% \mathrm{CI} 1.09 ; 1.60)$. This discrepancy might be due to the respective foods that have been summarised by the term 'refined grain products'. In the Nurses' Health Study (75,521 women), the consumption of refined grain products was not associated with the risk of diabetes (RR comparing extreme quintiles $=1.11 ; 95 \% \mathrm{CI}$ 0.94; 1.30) [Liu et al., 2000a] (LOE IIb). In the Finnish Mobile Clinic Health Examination Survey, the consumption of refined grain products was not significantly associated with the risk of type 2 diabetes mellitus (RR comparing extreme quartiles $=0.62 ; 95 \%$ CI $0.36 ; 1.06$ ) [Montonen et al., 2003] (LOE IIb).

The evidence regarding an association between the intake of polysaccharides and the risk of diabetes is insufficient.

\subsubsection{Dietary Fibre}

The relation between total dietary fibre intake and the occurrence of type 2 diabetes mellitus 2 has been investigated in several prospective cohort studies. Though an early analysis of the Nurses' Health Study (follow-up 1980-1986) did not show a significant association between dietary fibre intake and the risk of diabetes [Colditz et al., 1992] (LOE IIb), Salmeron et al. [1997b] (LOE IIb) observed an inverse association in this study after a follow-up period of 6 years (RR comparing extreme quintiles $=0.78 ; 95 \%$ CI $0.62 ; 0.88)$. It is important to mention that the investigation of Salmeron et al. was based on a later period (1986-1992) when a largely extended nutrition questionnaire was used. An inverse relation was also identified in the Iowa Women's Health Study (RR comparing extreme quintiles $=0.78 ; 95 \%$ CI $0.64 ; 0.96)$ [Meyer et al., 2000] (LOE IIb), the Finnish Mobile Clinic Health Examination Survey (RR comparing extreme quartiles = $0.51 ; 95 \%$ CI $0.26 ; 1.00$, p trend $=0.04$ ) [Montonen et al., 2003] (LOE IIb) and the British Regional Heart Study (RR dietary fibre intake $\leq 20 \mathrm{~g} /$ day vs. $>20 \mathrm{~g} /$ day: $1.47 ; 95 \% \mathrm{CI}$ 1.03 ; 2.11) [Wannamethee et al., 2009] (LOE IIb). In the Multiethnic Cohort in Hawaii, an inverse association was observed in men (RR comparing extreme quintiles = $0.75 ; 95 \%$ CI $0.67 ; 0.84$ ), but not in women (RR comparing extreme quintiles $=0.95 ; 95 \%$ CI $0.85 ; 1.06$ ) [Hopping et al., 2010] (LOE IIb). In contrast to these studies, the in- take of dietary fibre at baseline and its change during the 20-year follow-up in the Seven Countries Study including 338 men was not significantly associated with the risk of diabetes [Feskens et al., 1995] (LOE IIb). Likewise, there was no association in the Health Professionals Follow-Up Study, in which the comparison of extreme quintiles resulted in an RR of 0.98 (95\% CI $0.73 ; 1.33)$ [Salmeron et al., 1997a] (LOE IIb). In addition, higher total dietary fibre intake was not associated with the risk of diabetes in a study with Pima Indians (RR comparing extreme quartiles $=1.2$; 95\% CI 0.3; 4.9) [Williams et al., 2001] (LOE IIb), in the Atherosclerosis Risk in Communities Study $(\mathrm{RR}=0.999 ; 95 \%$ CI $0.987 ; 1.012$ for Whites and 0.998; 95\% CI 0.980; 1.017 for Afro-Americans, respectively) [Stevens et al., 2002] (LOE IIb), the Melbourne Collaborative Cohort Study (OR per $20 \mathrm{~g} / \mathrm{day}=0.93$; 95\% CI 0.73; 1.18) [Hodge et al., 2004] (LOE IIb), the Nurses' Health Study II (RR extreme quintile $=1.00 ; 95 \%$ CI $0.75 ; 1.34$ ) [Schulze et al., 2004a] (LOE IIb) and the EPIC-Potsdam Study (RR comparing extreme quintiles $=0.86 ; 95 \% \mathrm{CI}$ 0.65 ; 1.14) [Schulze et al., 2007b] (LOE IIb). In a post hoc analysis of the Finnish Diabetes Prevention Study, a reduced risk of diabetes with higher dietary fibre intake was observed; in this study, the participants of both the intervention and the control group were pooled so that the intervention design was dispersed [Lindstrom et al., 2006] (LOE IIb). RR for the highest quartile of dietary fibre intake $(>15.55 \mathrm{~g} / 1,000 \mathrm{kcal})$ compared with the lowest quartile $(<10.86 \mathrm{~g} / 1,000 \mathrm{kcal})$ was 0.38 (95\% CI 0.19 ; $0.77)$. However, no significant association was observed after adjustment for total fat content, which was an essential aspect of the intervention in addition to dietary fibre ( $\mathrm{RR}=0.88$; 95\% CI 0.68; 1.16). In an Australian cohort, no statistically significant association was found over 10 years of follow-up (RR per $5 \mathrm{~g} /$ day $=0.90 ; 95 \% \mathrm{CI}$ 0.79; 1.02) [Barclay et al., 2007] (LOE IIb).

Due to the varying results, the evidence regarding a lack of an association between total intake of dietary fibre and the risk of diabetes is judged as possible.

Differences between water-soluble and insoluble dietary fibre have only been investigated in a few studies. In the Iowa Women's Health Study, Meyer et al. [2000] (LOE IIb) observed a significant inverse association between insoluble dietary fibre and the risk of diabetes (RR comparing extreme quintiles $=0.75 ; 95 \%$ CI $0.61 ; 0.91$ ), but not for water-soluble dietary fibre ( $\mathrm{RR}=0.89 ; 95 \% \mathrm{CI}$ $0.73 ; 1.08)$. In the Finnish Mobile Clinic Health Examination Survey, both insoluble and water-soluble dietary fi- 
bre were inversely associated with the risk of diabetes, but this relation was only significant regarding insoluble dietary fibre (RR comparing extreme quartiles $=0.47$; $95 \%$ CI 0.25; 0.91) [Montonen et al., 2003] (LOE IIb). In the EPIC-Potsdam Study, Schulze et al. [2007b] (LOE IIb) observed no association with the risk of diabetes for either water-soluble or insoluble dietary fibre.

The evidence regarding a reduced risk of diabetes due to higher intake of insoluble dietary fibre is judged as insufficient. There is possible evidence that there is no association between water-soluble dietary fibre and the risk of diabetes.

A relatively large number of studies investigated dietary fibre separately according to their origin from cereal products, fruit and vegetables. A meta-analysis of 9 prospective cohort studies showed an inverse association between the intake of dietary fibre from cereal products and the risk of type 2 diabetes mellitus. Subjects with a relatively high intake (highest quintile or quartile) had a $\mathrm{RR}$ of 0.67 (95\% CI 0.62; 0.72) in comparison with subjects with low intake [Schulze et al., 2007b] (LOE IIa). Seven out of the 9 included studies showed a significant inverse association. These results are confirmed by a more recent cohort study (Black Women's Health Study) in which women with high intake of dietary fibre from cereal products showed reduced risk (RR comparing extreme quintiles $=0.82 ; 95 \%$ CI 0.70; 0.96) [Krishnan et al., 2007] (LOE IIb). In the Multiethnic Cohort as well, an inverse relation between the intake of dietary fibre from cereal products and the risk of diabetes was observed (RR comparing extreme quintiles in men $=0.91 ; 95 \%$ CI $0.82 ; 1.00 ; \mathrm{p}$ trend $=0.006$; RR in women $=0.88 ; 95 \%$ CI $0.79 ; 0.97 ; \mathrm{p}$ trend $=0.02$ ) [Hopping et al., 2010] (LOE IIb). In the British Regional Heart Study, there was no statistically significant association between low dietary fibre intake from cereal products and the risk of diabetes ( $R R \leq 6.9 \mathrm{~g} /$ day vs. $\geq 6.9$ g/day: 1.43 ; 95\% CI 1.00; 2.06) [Wannamethee et al., 2009] (LOE IIb). Barclay et al. [2007] (LOE IIb) did not find an association in an Australian cohort either $(\mathrm{RR}$ per $5 \mathrm{~g} / \mathrm{day}=0.96$; $95 \% \mathrm{CI} 0.78 ; 1.20)$. It is confirmed by another meta-analysis which investigated the consumption of whole-grain products that the intake of dietary fibre from cereal products lowers the risk of diabetes. Combined analysis of 6 cohort studies showed a reduced risk of type 2 diabetes mellitus with each increase in whole-grain product intake by 2 portions/day ( $\mathrm{RR}=$ 0.79; 95\% CI 0.72; 0.87) [de Munter et al., 2007] (LOE IIa). A prospective cohort study (Physicians' Health Study, $\mathrm{n}=$
$21,152)$ published after this meta-analysis also observed a lowering of risk with higher whole-grain product intake. Men who consumed whole-grain breakfast cereals daily had an RR of 0.60 (95\% CI 0.50; 0.71) compared with men who never consumed such cereal products [Kochar et al., 2007] (LOE IIb). In the EPIC-Potsdam Study, the consumption of whole-grain bread was inversely associated with the risk of diabetes (RR per $50 \mathrm{~g} / \mathrm{day}=0.918 ; 95 \% \mathrm{CI}$ $0.855 ; 0.986$ ) [Schulze et al., 2007a] (LOE IIb). However, this association does not seem to apply for carriers of a certain gene variant of TCF7L2 [Fisher et al., 2009] (LOE IIb). A Cochrane Review by Priebe et al. [2008] provides an overview of studies on the association between the intake of dietary fibre from cereal products and the risk of diabetes. The studies that are relevant for the present guideline have been considered in this chapter.

The prospective cohort studies indicate with high consistency that high intake of whole-grain products or dietary fibre from cereal products, respectively, causes a lower risk of diabetes. The evidence regarding this association is judged as probable.

According to a meta-analysis of prospective cohort studies, the intake of dietary fibre from fruit (8 studies; $\mathrm{RR}=0.96 ; 95 \%$ CI $0.88 ; 1.04)$ or vegetables (7 studies; $\mathrm{RR}=1.04 ; 95 \%$ CI $0.94 ; 1.15)$ was not associated with the risk of developing type 2 diabetes mellitus [Schulze et al., 2007b] (LOE IIa). Consistently, there was no association observed in all the studies included in this meta-analysis. Barclay et al. [2007] (LOE IIb) did not observe an association regarding dietary fibre from fruit in an Australian cohort either (RR per $5 \mathrm{~g} / \mathrm{day}=0.94 ; 95 \%$ CI $0.78 ; 1.15$ ); however, there was an inverse association regarding dietary fibre from vegetables (RR per $5 \mathrm{~g} / \mathrm{day}=0.76$; $95 \% \mathrm{CI}$ 0.57; 0.99). In the Multiethnic Cohort in Hawaii, Hopping et al. [2010] (LOE IIb) reported an inverse association between the intake of dietary fibre from vegetables and the risk of diabetes in men (RR comparing extreme quintiles $=0.78$; $95 \%$ CI $0.69 ; 0.88)$, but not in women $(\mathrm{RR}=$ $0.96 ; 95 \%$ CI $0.87 ; 1.08)$. In the same cohort, dietary fibre from fruit was not associated with the risk of diabetes in either men (RR comparing extreme quintiles $=0.93 ; 95 \%$ CI $0.84 ; 1.02)$ or women ( $\mathrm{RR}=0.95 ; 95 \%$ CI $0.85 ; 1.06)$. Low intake of dietary fibre from vegetables was not significantly associated with the risk of diabetes in the British Regional Heart Study (RR $\leq 11.3$ vs. $>11.3$ g/day $=$ $1.40 ; 95 \%$ CI 0.98; 1.98) [Wannamethee et al., 2009] (LOE IIb). 
The evidence regarding a lack of association between the intake of dietary fibre from fruit and vegetables and the risk of diabetes is judged as probable.

\subsection{Glycaemic Index and Glycaemic Load}

The results of several prospective cohort studies on GI and GL were summarised in 2008 by a meta-analysis. For this, Barclay et al. [2008] (LOE IIa) included 7 studies on GI and 8 studies on GL. The meta-analysis resulted in a significant positive association for both GI (RR comparing extreme quantiles $=1.20 ; 95 \%$ CI $1.04 ; 1.38$ ) and for GL (1.16; 95\% CI 1.04; 1.28). However, 1 of the included studies had gestational diabetes as endpoint [Zhang et al., 2006]. Another study investigated pancreatic carcinoma as primary endpoint; it remained unclear which modelling was the basis for the association between GL and diabetes that has been reported in the discussion [Patel et al., 2007] (LOE IIb). Furthermore, it is noticeable that the included studies gave quite heterogeneous results, but these have not been quantified by the authors. Positive associations have been observed in particular in the Nurses' Health Study II [Schulze et al., 2004a] (LOE IIb) as well as in the Health Professionals Follow-Up Study [Salmeron et al., 1997a] (LOE IIb), while these kinds of associations could not be found in the Nurses' Health Study [Salmeron et al., 1997b] (LOE IIb), the Iowa Women's Health Study [Meyer et al., 2000] (LOE IIb) and the Atherosclerosis Risk in Communities Study [Stevens et al., 2002] (LOE IIb). In the Melbourne Collaborative Cohort Study, a positive association regarding GI was observed, but there was no evidence of an association between GL and the risk of diabetes [Hodge et al., 2004] (LOE IIb).

There are further studies available whose results have not been included in the meta-analysis, partly due to the later date of publication. In the Insulin Resistance Atherosclerosis Study, no associations between GI and GL and the risk of diabetes have been found. However, GI in subjects without abdominal obesity (waist circumference $\leq 102 \mathrm{~cm}$ for men and $\leq 88 \mathrm{~cm}$ for women) and subjects with an increasing waist circumference during follow-up was associated with an increased risk; but this association was only statistically significant in the latter group $(\mathrm{p}=$ 0.04) [Schulz et al., 2006] (LOE IIb). In the Black Women's Health Study (40,078 women, 8 years of follow-up), a higher GI was associated with a higher risk of diabetes (RR comparing extreme quintiles $=1.23$; 95\% CI 1.05; 1.44). Regarding GL, this tendency was seen as well; however, this relation was not statistically significant. The RR for the comparison of extreme quintiles was 1.22 (95\% CI $0.98 ; 1.51, \mathrm{p}$ trend $=0.06$ ) [Krishnan et al., 2007] (LOE IIb). In the Shanghai Women's Health Study, Villegas et al. [2007] (LOE IIb) observed a positive association regarding GI (RR comparing extreme quintiles $=1.21 ; 95 \%$ CI $1.03 ; 1.43)$ and GL $(1.34 ; 1.13 ; 1.58)$. Hu et al. [2001] (LOE IIb) as well as Halton et al. [2008] (LOE IIb) reported a strong positive association between GL and the risk of diabetes after 20 years of follow-up in the Nurses' Health Study ( $\mathrm{n}=85,059)$. In the current evaluation by Halton et al. [2008] (LOE IIb), RR comparing women in the highest decile was 2.47 (95\% CI 1.75; 3.47) compared with women in the lowest decile of GL. However, intake of total energy, protein, saturated fatty acids, polyunsaturated fatty acids and trans-fatty acids was adjusted for at the same time. As such a model practically excludes a GL increase by means of a higher carbohydrate proportion in the diet, these results rather have to be interpreted as an association between GI (and not GL) and diabetes. In the Multiethnic Cohort, higher GL was positively associated with the risk of diabetes in women (RR comparing extreme quintiles $=1.41 ; 95 \%$ CI 1.15; 1.73), but not in men $(\mathrm{RR}=1.16$; 95\% CI 0.99; 1.36) [Hopping et al., 2010] (LOE IIb). In contrast to these studies, Mosdøl et al. [2007] (LOE IIb) could not identify an increased risk of diabetes for either higher GI or higher GL in the Whitehall II Study. The RRs for comparison of extreme terciles were $0.94(95 \%$ CI $0.71 ; 1.23)$ for GI and 0.80 (95\% CI $0.51 ; 1.26)$ for GL, respectively. Likewise, in the Health, Aging, and Body Composition Study $(\mathrm{n}=1,898)$, there were no associations between GI (RR comparing extreme quintiles = 1.0 ; 95\% CI $0.5 ; 2.0)$ and GL $(1.3 ; 95 \%$ CI $0.6 ; 2.7)$, respectively, and type 2 diabetes mellitus [Sahyoun et al., 2008] (LOE IIb) during a follow-up period of 4 years. A metaanalysis published in 2010 resulted in an RR of 1.58 (95\% CI 1.30; 1.86) for GL [Liu and Chou, 2010] (LOE IIa). This analysis included a more recent cohort study (EPIC-NL), in which a positive association has been observed [Sluijs et al., 2010] (LOE IIb). However, the results of the Iowa Women's Health Study [Meyer et al., 2000] (LOE IIb) and of the Multiethnic Cohort Study [Hopping et al., 2010] (LOE IIb) are missing in this analysis. The result has clearly been influenced by the study of Halton et al. [2008] (LOE IIb), in which grouping (deciles) differed from other studies and which does not allow an unambigious interpretation of the results. Besides, results from the Nurses' Health Study are included into the meta-analysis twice [Salmeron et al., 1997b] (LOE IIb); [Halton et al., 2008] (LOE IIb). 
Although a meta-analysis which includes some of the studies which have been published until then shows a higher risk of diabetes with higher GI, the evidence regarding a positive association for GI is judged as possible, due to the heterogeneity of the results of all studies. Two meta-analyses indicate a positive association regarding GL. However, most of the studies indicate a lack of an association and the meta-analyses show methodological weaknesses. The evidence regarding a lack of association is judged as possible, due to the partial inconsistency of the study results.

\subsection{Need for Research regarding Type 2 Diabetes Mellitus}

The relevance of the absolute carbohydrate intake regarding the risk of diabetes is unknown so far. As some prospective cohort studies have investigated associations between the relative proportion of carbohydrates in total energy intake, it would be possible and desirable to analyse this question in the available studies to create a reliable database.

The database regarding the relevance of polysaccharides, sucrose, glucose and fructose for the risk of diabetes is unsteady as well. The results of the available studies are too inconsistent, maybe due to methodological problems. As only a few studies have investigated these kinds of carbohydrates, it is desirable to analyse available prospective studies regarding this aspect.

Although some studies have investigated relations between GI and GL and the risk of diabetes, results have been very heterogeneous so far. Here there are methodological problems as well, especially regarding the characterisation of the participants' diet in cohort studies. The significance of studies depends to a great extent on the quality of the GI tables being used. Efforts should be made to improve the quality of data from available studies. Besides, studies vary with regard to the nutrition survey instruments that have been used and which usually have not been designed specifically for the determination of GI or GL.

There is also need for research regarding the question of how the kind of dietary fibre modifies the risk of diabetes, particularly as there is a considerable preventive potential. There is probable evidence that whole-grain products or dietary fibre from cereal products, respectively, reduce the risk of diabetes. Intervention studies on cereal products in particular would be highly desirable to prove causation of this association without any doubt.

\section{Quantity and Quality of Carbohydrate Intake and Primary Prevention of Dyslipoproteinaemia}

For healthy individuals, it is a goal to avoid the occurrence of dyslipoproteinaemia caused by environmental factors. In inherited forms of dyslipoproteinaemia, however, primary prevention can only achieve a delayed and/ or attenuated disease manifestation. Intervention studies on long-term primary prevention of primary dyslipoproteinaemia depending on the intake of carbohydrates, mono- and disaccharides, dietary fibre or GI and GL, respectively, could not be found.

\subsection{Proportion of Carbohydrates}

Foods that are naturally rich in carbohydrates usually are also rich in dietary fibre. Therefore, changes in carbohydrate intake are also associated with changes in dietary fibre intake and vice versa. In this subparagraph (5.1), only studies are mentioned in which dietary fibre intake rises by up to $7 \mathrm{~g} /$ day with the increase in carbohydrate intake (arbitrarily chosen limiting value). Studies with a simultaneous dietary fibre intake increase of more than $7 \mathrm{~g} /$ day are presented in subparagraph 5.3.1 Dietary Fibre.

Total and LDL Cholesterol

In a 2.5-year cohort study including 1,182 nine-yearold children with a mean dietary fat percentage of 33 $\mathrm{EN} \%$ and a dietary fibre intake of $15 \mathrm{~g} /$ day, an increase in carbohydrate intake was associated with a lowering of the total cholesterol concentration $(\beta=-0.021, p<0.02)$ [Nicklas et al., 2002] (LOE IIb). A cohort study including 157 men and women with an observation period of 9 years showed a decrease in plasma levels of LDL cholesterol (men $-0.028 \mathrm{mmol} / \mathrm{l}$; women $-0.022 \mathrm{mmol} / \mathrm{l} ; \mathrm{p}<$ 0.05 ) with an increase in carbohydrate intake (from 46 to $53 \mathrm{EN} \%$ in women, from 44 to $50 \mathrm{EN} \%$ in men). In women, the total cholesterol concentration was lowered by $0.039 \mathrm{mmol} / \mathrm{l}$ and in men by $0.059 \mathrm{mmol} / \mathrm{l}(\mathrm{p}<0.05 \mathrm{each}$ ). Body weight decreased by an average of not more than $1 \mathrm{~kg}$ [Garry et al., 1992] (LOE IIb). Ma et al. [2006] (LOE IIb) observed in a cohort study including 574 men and women that after 1 year, the carbohydrate proportion was not associated with total and LDL cholesterol in either cross-sectional or longitudinal analyses. In the Multiple Risk Factor Intervention Trial including 6,438 overweight men and an intervention period of 6 years, energy intake was lowered in the intervention group due to the speci- 
fied selection of foods; therefore, only the findings in the control group could be used. In the course of the study, a significant inverse association between carbohydrate intake and plasma total and LDL cholesterol $(\mathrm{p}<0.001$ and $p<0.01$, respectively) was shown [Tillotson et al., 1997a] (LOE IIb).

In a prospective cohort study including 2,909 subjects, LDL cholesterol levels did not differ significantly after 10 years between white and black men and women with higher carbohydrate intake (48 EN\%) and those with lower carbohydrate intake (36 EN\%). Dietary fibre intake was between $5.9 \mathrm{~g} / \mathrm{MJ}$ and $10.5 \mathrm{~g} / \mathrm{MJ}$ [Ludwig et al., 1999] (LOE IIb). Twisk et al. [1997] (LOE IIb) did not find an association in adolescents between carbohydrate intake and total or LDL cholesterol. In the Northern Ireland Young Hearts Project including 459 12-year-old girls and boys, no association between higher carbohydrate intake (53 EN\%) and the plasma total cholesterol concentration was found over the course of 3 years [Boreham et al., 1999] (LOE IIb).

According to a meta-analysis of 72 intervention studies including 129 groups of men and women in 395 experiments with a median duration of 1 month, replacement of $10 \mathrm{EN} \%$ of total fat intake by complex carbohydrates resulted in a decrease in plasma levels of total $[-0.20(0.05) \mathrm{mmol} / \mathrm{l}]$ and LDL cholesterol $[-0.12$ (0.06) $\mathrm{mmol} / \mathrm{l}]$; replacement of $10 \mathrm{EN} \%$ of saturated fatty acids by complex carbohydrates also led to a decrease in total $[-0.52(0.03) \mathrm{mmol} / \mathrm{l}]$ and LDL cholesterol $[-0.36(0.05) \mathrm{mmol} / \mathrm{l}]$. The isocaloric substitution of $5 \mathrm{EN} \%$ of complex carbohydrates with polyunsaturated fatty acids resulted in a further decrease in the total cholesterol concentration by $0.13(0.02) \mathrm{mmol} / \mathrm{l}$ and of the LDL cholesterol concentration by $0.11(0.03) \mathrm{mmol} / \mathrm{l}$. Substitution of complex carbohydrates with monounsaturated fatty acids did not have a significant influence on the total and LDL cholesterol concentrations [Clarke et al., 1997] (LOE Ia).

According to a meta-analysis including 628 men and women from 27 controlled intervention studies with a duration of 14-91 days, the replacement of 1 EN\% of carbohydrate intake by saturated fatty acids resulted in a calculated increase in the total $(\beta=0.039)$ and LDL cholesterol concentrations $(\beta=0.033)$ ( $p<0.001$ each). The replacement of 1 EN\% of carbohydrate intake by monounsaturated fatty acids led to a non-significant decrease in the total $(\beta=0.003, p=0.342)$ and LDL cholesterol concentrations $(\beta=0.006, p=0.114)$, and the replacement of $1 \mathrm{EN} \%$ of carbohydrates by polyunsaturated fatty acids resulted in a significant decrease in the total $(\beta=0.015$, $p=0.001)$ and LDL cholesterol concentrations $(\beta=0.014$, $\mathrm{p}=0.002$ ) [Mensink and Katan, 1992] (LOE Ia). According to another meta-analysis performed by the same workgroup including 1,672 men and women from 60 controlled intervention studies with a duration of 13-91 days and a mean fat intake of $34 \mathrm{EN} \%$, the replacement of $1 \mathrm{EN} \%$ of carbohydrate intake by saturated fatty acids resulted in a calculated increase in the total $(\beta=0.036)$ and LDL cholesterol concentrations $(\beta=0.032)(p<0.001$ each). The replacement of $1 \mathrm{EN} \%$ of carbohydrate intake by monounsaturated fatty acids led to a non-significant decrease in the total cholesterol concentration $(\beta=-0.006$, $\mathrm{p}=0.061)$ and to a significant decrease in the LDL cholesterol concentration $(\beta=-0.009, p=0.004)$; the replacement of $1 \mathrm{EN} \%$ of carbohydrate intake by polyunsaturated fatty acids led to a decrease in the total cholesterol concentration $(\beta=-0.021, \mathrm{p}<0.001)$ and LDL cholesterol concentration $(\beta=-0.019, \mathrm{p}<0.001)$ [Mensink et al., 2003] (LOE Ia). In another meta-analysis of 30 intervention studies ( 24 crossover, 6 parallel design studies) with a duration of 14-84 days including 1,213 women and men, among them 90 diabetics, the effects of diets with $18-30$ EN\% fat were compared with the effects of diets with $30-50$ EN\% fat. The difference in fat content was mainly achieved by doubling the content of monounsaturated fatty acids (on average, from 11.4 to $23.6 \mathrm{EN} \%$ ) with otherwise unchanged fatty acid composition. Replacement of carbohydrates by monounsaturated fatty acids in the diets with $30-50 \mathrm{EN} \%$ fat resulted in a non-significant reduction in the plasma total and LDL cholesterol concentrations [Cao et al., 2009] (LOE Ia).

In addition to these meta-analyses, individual studies are available. In a 1-year intervention study including 146 men and women, an increase in carbohydrate intake (from 50 to $55 \mathrm{EN} \%$ ) and dietary fibre intake (from 19 to $25 \mathrm{~g} /$ day) resulted in a significant reduction in the concentrations of total and LDL cholesterol, respectively, in comparison to the control group. However, in the control group similar changes in the plasma lipids were observed as well. This seems to be due to an increased nutritional awareness, though the eating habits were not supposed to be changed. Body weight decreased by about $1 \mathrm{~kg}$ [Anderson et al., 1992] (LOE Ib). An intervention study with 32 very active and well-trained endurance athletes over 12 weeks with an increase in carbohydrate intake from 55 to $69 \mathrm{EN} \%$ and in dietary fibre intake from 40 to $46 \mathrm{~g} /$ day at constant body weight showed a significant increase in the total cholesterol concentration (from 4.42 to $4.62 \mathrm{mmol} / \mathrm{l} ; \mathrm{p}<0.05)$ and a non-significant increase in the LDL cholesterol concentration (from 2.73 to 2.84 
$\mathrm{mmol} / \mathrm{l})$. There were no significant differences in comparison to a parallel intervention group with a diet rich in fat (47 EN\% fat) [Brown et al., 1998] (LOE Ib). In the 1-year intervention study by Kasim et al. [1993] (LOE Ib) including 72 women, the increase in carbohydrate intake by $11 \mathrm{EN} \%$ (and at the same time a slight increase in dietary fibre intake by $4 \mathrm{~g} /$ day) was accompanied by an unchanged total cholesterol concentration but a lower LDL cholesterol concentration. However, at the end of this study, a weight loss of $3.4 \mathrm{~kg}$ in the test group and of $0.8 \mathrm{~kg}$ in the control group was observed. Sacks et al. [1986] (LOE Ic) advised 22 men and women to consume a diet high in carbohydrates (increase in carbohydrate intake from 50 to $62 \mathrm{EN} \%$; in dietary fibre intake from 24 to $27 \mathrm{~g} /$ day) and observed significant decreases in the total and LDL cholesterol concentrations after 3 months, while the participants kept a constant body weight. However, this study had no control group. Starting with a carbohydrate percentage of $51 \mathrm{EN} \%, 36$ adolescents received a diet rich in carbohydrates for 3 months (60 EN\%) by Vartiainen et al. [1986] (LOE Ic). There was no control group either. The increase in dietary fibre intake was less than $5 \mathrm{~g} /$ day. Body weight did not change. The diet with increased carbohydrate intake lowered the total cholesterol concentration (from 4.94 to $4.18 \mathrm{mmol} / \mathrm{l}, \mathrm{p}<$ 0.001 ). An intervention group without control group by Kasim-Karakas et al. [2000] (LOE Ic) showed a significant decrease in the total cholesterol concentration ( $\mathrm{p}<$ 0.05 ), but not in the LDL cholesterol concentration after a 1-year diet rich in carbohydrates (67 EN\%). The increase in dietary fibre intake was less than $5 \mathrm{~g} /$ day. The study resulted in a weight loss of $6 \mathrm{~kg}$.

In an intervention study including 200 women over 1 year, an increase in carbohydrate intake from 44 to 52 EN\%, at constant body weight, did not result in a significant decrease in the total cholesterol concentration [Boyd et al., 1990] (LOE Ib). In the CARMEN Study, 316 overweight adults received a diet rich in mono- and disaccharides or rich in complex carbohydrates (total carbohydrate intake 55 and $52 \mathrm{EN} \%$, respectively) for 6 months compared with a control group (45 EN\%). There was no restriction of energy intake. Dietary fibre intake changed only slightly ( $<2$ g/day). Body weight was reduced by 1.7 $\mathrm{kg}$ with the diet rich in mono- and disaccharides and by $2.6 \mathrm{~kg}$ with the diet rich in complex carbohydrates compared with the control group. Regarding plasma lipoproteins, it did not make a significant difference whether the fat in the low-fat diets was substituted with mono- and disaccharides or with complex carbohydrates, compared with baseline values and the control group [Saris et al.,
2000] (LOE Ib). In an intervention study including 644 children over several years, the association between carbohydrate intake and serum lipids as well as in dependence of the apoE phenotype was investigated. After adjustment, there was no association between the total and LDL cholesterol concentrations and carbohydrate intake [Ruottinen et al., 2009] (LOE Ib).

In the intervention study by Krauss et al. [2006], men with a BMI between 26 and 35 were divided into 4 subsets and assigned to a diet with $54 \mathrm{EN} \%$ (basal diet), with 39 EN\%, or with $26 \mathrm{EN} \%$ carbohydrates and a low content of saturated fatty acids (7-9 EN\%), or with 26 EN\% carbohydrates and a higher content of saturated fatty acids (15 EN\%). After 1 week of consuming a basal diet that all participants had to stick to and 3 weeks of diet with 39 EN\% carbohydrates and 7-9 EN\% saturated fatty acids, the total cholesterol concentration was lowered by $9.4 \mathrm{mg} /$ $\mathrm{dl}$ and the LDL cholesterol concentration by $0.6 \mathrm{mg} / \mathrm{dl}$. However, the difference was not significant. The diet with the $26 \mathrm{EN} \%$-carbohydrate and low saturated fatty acids content (7-9 EN\%) significantly lowered the total cholesterol concentration by $21.4 \mathrm{mg} / \mathrm{dl}$ and non-significantly lowered the LDL cholesterol by $11.2 \mathrm{mg} / \mathrm{dl}$. The diet with the $26 \mathrm{EN} \%$-carbohydrate and higher saturated fatty acids content (15 EN\%) lowered the total cholesterol concentration by $10.7 \mathrm{mg} / \mathrm{dl}$ and the LDL cholesterol concentration by $0.7 \mathrm{mg} / \mathrm{dl}$. These differences were not significant. Regarding the $39 \mathrm{EN} \%$ - and $26 \mathrm{EN} \%$-carbohydrate diets, the protein percentage in the diet increased from 15 to $29 \mathrm{EN} \%$. After subsequent weight reduction over a period of 5 weeks, total and LDL cholesterol concentrations had further decreased with the 54 EN\%-carbohydrate diet; however, this association was not significant. Regarding the $39 \mathrm{EN} \%$-carbohydrate diet, the total and LDL cholesterol concentrations decreased slightly, but not significantly. In contrast, the total and LDL cholesterol concentrations significantly increased again with the 26 EN\%-carbohydrate and low saturated fatty acids content diet (total cholesterol concentration $+7 \mathrm{mg} / \mathrm{dl}$, LDL cholesterol concentration $+4.3 \mathrm{mg} / \mathrm{dl}$ ), while the increase with the 26 EN\%-carbohydrate and higher saturated fatty acids content diet was not significant [Krauss et al., 2006] (LOE Ib).

There is convincing evidence that a higher carbohydrate proportion in the diet at the expense of total fat or saturated fatty acids intake lowers the plasma concentrations of total and LDL cholesterol. An increase in carbohydrate proportion at the expense of polyunsaturated fatty acids results with convincing evidence in an increase 
in the plasma concentrations of total and LDL cholesterol. This judgement is in particular derived from the consistent results of 3 large meta-analyses of intervention stud$i e s$. The present studies provide convincing evidence regarding a lack of an association between the exchange of carbohydrates for monounsaturated fatty acids and the plasma concentrations of total and LDL cholesterol.

\section{HDL Cholesterol}

Garry et al. [1992] (LOE IIb) and Nicklas et al. [2002] (LOE IIb) observed a decrease in the HDL cholesterol concentration with an increase in carbohydrate intake [-0.022 (women), -0.028 (men) $\mathrm{mmol} / \mathrm{l} ; \mathrm{p}<0.05$ or -0.010 $\mathrm{mmol} / \mathrm{l} ; \mathrm{p}<0.005]$. A cohort study, which lasted for 15 years and included 181 adolescents, showed a significant association between an increased carbohydrate intake (44-46 EN\%) and a lowered HDL cholesterol concentration [Twisk et al., 1997] (LOE IIb). In the study by Tillotson et al. [1997a] (LOE IIb), there was a significant inverse association in the control group between carbohydrate intake and the HDL cholesterol concentration. The carbohydrate proportion (EN\%) was significantly inversely associated with the plasma HDL cholesterol concentration in the cohort study by Ma et al. [2006] (LOE IIb) in cross-sectional and in longitudinal analyses.

Boreham et al. [1999] (LOE IIb) found an inverse association between carbohydrate intake and the HDL cholesterol concentration only in girls $(\beta=-0.22, p=0.014)$, but not in boys. In another prospective cohort study including 2,909 subjects, plasma HDL cholesterol levels did not differ significantly after 10 years between white and black men and women with higher carbohydrate intake and those with lower carbohydrate intake [Ludwig et al., 1999] (LOE IIb).

In a meta-analysis of 72 intervention studies with multivariate analysis, the replacement of $10 \mathrm{EN} \%$ of total fat by complex carbohydrates resulted in a decrease in plasma HDL cholesterol levels $[-0.10(0.02) \mathrm{mmol} / \mathrm{l}]$, and the replacement of $10 \mathrm{EN} \%$ of saturated fat by complex carbohydrates led to a decrease in the HDL cholesterol concentration as well $[-0.13(0.02) \mathrm{mmol} / \mathrm{l}]$. Isocaloric substitution of $5 \mathrm{EN} \%$ of complex carbohydrates with polyunsaturated fatty acids resulted in an increase in the HDL cholesterol concentration by $0.03(0.01) \mathrm{mmol} / \mathrm{l}$. An equivalent substitution of complex carbohydrates with monounsaturated fatty acids led to an increase in the plasma HDL cholesterol concentration [Clarke et al., 1997] (LOE Ia).

According to a meta-analysis [Mensink and Katan, 1992] (LOE Ia) including 628 men and women from 27 controlled intervention studies with a duration of 14-91 days, the replacement of $1 \mathrm{EN} \%$ of carbohydrate intake by saturated fatty acids resulted in a calculated increase ( $\beta=0.012, p<0.001)$ in the plasma concentration of HDL cholesterol; the replacement by monounsaturated fatty acids $(\beta=0.009, \mathrm{p}<0.001)$ and by polyunsaturated fatty acids $(\beta=0.007, p<0.002)$ resulted in an increase as well. In another meta-analysis performed by the same workgroup including 1,672 men and women from 60 controlled intervention studies with a duration of 13-91 days and a mean fat intake of $34 \mathrm{EN} \%$, the replacement of $1 \mathrm{EN} \%$ of carbohydrate intake by saturated fatty acids resulted in an increase in the plasma HDL concentration $(\beta=-0.010, p<0.001)$, the replacement by monounsaturated fatty acids $(\beta=0.008, \mathrm{p}<0.001)$ and by polyunsaturated fatty acids $(\beta=0.006, p<0.001)$ resulted in an increase as well. The total cholesterol/HDL cholesterol quotient did not change significantly, when saturated fatty acids were replaced by carbohydrates. But the quotient decreased significantly when carbohydrates or saturated fatty acids were replaced by mono- or cis-polyunsaturated fatty acids [Mensink et al., 2003] (LOE Ia).

In another meta-analysis of 30 intervention studies ( 24 crossover, 6 parallel design studies) including 1,213 women and men, among them 90 diabetics, with a duration of 14-84 days, the effects of diets with 18-30 EN\% fat (mean $26 \mathrm{EN} \%$ fat) were compared with the effects of diets with 30-50 EN\% fat (mean 39 EN\% fat). The difference in fat content was mainly achieved by doubling the content of monounsaturated fatty acids (from 11.4 to $23.6 \mathrm{EN} \%$ ) with otherwise unchanged fatty acid composition. The exchange of carbohydrates for unsaturated fatty acids in the diets with 30-50 EN\% fat resulted in a significant increase ( $p<0.00001)$ in the plasma HDL cholesterol concentration. Regression analysis indicated a significant positive association only between the increase in total fat intake and the HDL cholesterol concentration. The total cholesterol/HDL cholesterol quotient was more reduced in the diets with $30-50$ EN\% $(-0.36)$ than in the diets with 18-30 EN\% fat (-0.06). There was a significant difference between the quotients ( $\mathrm{p}=0.001$ ) [Cao et al., 2009] (LOE Ia).

In addition to these meta-analyses, individual studies are available. In the intervention study by Kasim et al. [1993] (LOE Ib), increased carbohydrate intake (and at the same time slightly increased dietary fibre intake by $4 \mathrm{~g}$ /day) was accompanied by a lower plasma HDL cholesterol concentration (from 1.56 to $1.44 \mathrm{mmol} / \mathrm{l}, \mathrm{p}<0.05$ ). In the study by Sacks et al. [1986] (LOE Ic), a diet high in carbohydrates resulted in a significant decrease in the HDL cholesterol concentration as well. The intervention 
study by Vartiainen et al. [1986] (LOE Ic) showed a decrease in the HDL cholesterol concentration (from 1.45 to $1.20 \mathrm{mmol} / \mathrm{l}, \mathrm{p}<0.001$ ) with a diet rich in carbohydrates. A high-carbohydrate diet significantly lowered the plasma HDL concentration in postmenopausal women (from 1.76 to $1.45 \mathrm{mmol} / \mathrm{l}, \mathrm{p}<0.001)$ in the intervention study by Kasim-Karakas et al. [2000] (LOE Ic). In an intervention study including 644 children over several years, the association between carbohydrate intake and serum lipids as well as in dependence of the apoE phenotype was investigated. Even after adjustment, there was a significant inverse association between the HDL cholesterol concentration and carbohydrate intake $(\beta=-0.0058, \mathrm{p}<0.001)$ [Ruottinen et al., 2009] (LOE Ib).

In the intervention study by Anderson et al. [1992] (LOE Ib), higher carbohydrate and dietary fibre intake did not have a significant influence on the HDL cholesterol concentration. In the intervention study by Brown et al. [1998] (LOE Ib), there was no significant change of the plasma HDL cholesterol concentration with a highcarbohydrate diet.

In an intervention study with 4 groups consisting of 45 healthy subjects each and $54 \mathrm{EN} \%, 39 \mathrm{EN} \%$ as well as 26 EN\% carbohydrates (the latter with different proportions of saturated fatty acids), HDL cholesterol concentrations remained largely constant with all tested diets and were non-significantly increased by $2 \mathrm{mg} / \mathrm{dl}$ after weight reduction. The total cholesterol/HDL cholesterol quotient decreased much more with the diets containing $26 \mathrm{EN} \%$ carbohydrates (significantly with 7-9 EN\% saturated fatty acids) than with the diet containing $39 \mathrm{EN} \%$. The reduction in the HDL cholesterol concentration with 54 EN\% carbohydrate was marginal, but after weight reduction it was higher than with $39 \mathrm{EN} \%$ and $26 \mathrm{EN} \%$ carbohydrates, while the reduction in the HDL cholesterol concentration with the $26 \mathrm{EN} \%$-carbohydrate diet was marginal, but significant (p < 0.01) [Krauss et al., 2006] (LOE Ib).

There is convincing evidence that a higher carbohydrate proportion in the diet at the expense of total fat or saturated fatty acids intake lowers the plasma concentration of HDL cholesterol. There is convincing evidence that a higher carbohydrate proportion in the diet at the expense of mono- or polyunsaturated fatty acids intake lowers the plasma concentration of HDL cholesterol.

The present studies provide convincing evidence that the total cholesterol/HDL cholesterol quotient remains unchanged with the exchange of saturated fatty acids for carbohydrates and is lowered with the exchange of carbohydrates for mono- or polyunsaturated fatty acids.

\section{Triglycerides}

In a cohort study by Ma et al. [2006] (LOE IIb) including 574 healthy men and women, the carbohydrate proportion was significantly associated with the triglyceride concentration in the cross-sectional analysis $(\beta=0.09$, $p=0.047$ ), but not in the longitudinal analysis. There was no significant association between carbohydrate intake and the triglyceride concentration in the cohort study by Garry et al. [1992] (LOE IIb) in 157 men and women after a 9-year follow-up period.

According to the study by Millen et al. [2006] (LOE IIb), a higher carbohydrate and dietary fibre intake is not associated with a higher triglyceride concentration. In another prospective cohort study including 2,909 subjects, triglyceride concentrations did not differ significantly after 10 years between white and black men and women with higher carbohydrate intake and those with lower carbohydrate intake [Ludwig et al., 1999] (LOE IIb). The quantity of carbohydrate intake did not influence the plasma triglyceride concentration in the study by Tillotson et al. [Tillotson et al., 1997a] (LOE IIb).

According to a meta-analysis including 628 men and women from 27 controlled intervention studies with a duration of 14 days, the replacement of $1 \mathrm{EN} \%$ of carbohydrate intake by saturated fatty acids resulted in a calculated decrease in plasma triglyceride concentrations $(\beta=-0.025 ; p<0.001)$, likewise this is valid for a replacement by monounsaturated fatty acids $(\beta=-0.022$; $\mathrm{p}<$ $0.001)$ as well as a replacement by polyunsaturated fatty acids $(\beta=-0.028 ; p<0.001)$ [Mensink and Katan, 1992] (LOE Ia). In a meta-analysis including 1,672 men and women from 60 controlled intervention studies with a duration of 13-91 days and a mean fat intake of $34 \mathrm{EN} \%$, the replacement of $1 \mathrm{EN} \%$ of carbohydrate intake by saturated fatty acids resulted in a decrease in plasma triglyceride concentrations $(\beta=-0.021, p<0.001)$, likewise this is valid for a replacement by monounsaturated fatty acids $(\beta=-0.019, p<0.001)$ as well as a replacement by polyunsaturated fatty acids $(\beta=-0.026, p<0.001)$ [Mensink et al., 2003] (LOE Ia). In another meta-analysis of 30 intervention studies ( 24 crossover, 6 parallel design studies) including 1,213 women and men, among them 90 diabetics, with a duration of 14-84 days, the effects of diets containing 18-30 EN\% fat (mean 26 EN\% fat) were compared with the effects of diets containing 30-50 EN\% fat (mean $39 \mathrm{EN} \%$ fat). The difference in fat content was mainly achieved by doubling the content of monounsaturated fatty acids (from 11.4 to 23.6 EN\%) with otherwise unchanged fatty acid composition. Replacement of carbohydrates by monounsaturated fatty acids in the diets with 
$30-50 \mathrm{EN} \%$ fat resulted in a significant $(\mathrm{p}<0.00001)$ reduction in plasma triglyceride concentrations in the subjects without diabetes. Regression analysis indicated a significant association only between the increase in total fat intake and the decrease in triglyceride concentrations [Cao et al., 2009] (LOE Ia).

In addition to these meta-analyses, individual studies are available. In the intervention study by Brown et al. [1998] (LOE Ib), the increase in carbohydrate intake (from 55 to $70 \mathrm{EN} \%$ ) resulted in a significant increase in the plasma triglyceride concentration (from 1.04 to $1.28 \mathrm{mmol} / \mathrm{l}$, $\mathrm{p}<0.001$ ). In an intervention study including 644 children, the association between carbohydrate intake and serum lipids as well as in dependence of the apoE phenotype was investigated for several years. Even after adjustment, there was a significant positive association between triglyceride concentration and carbohydrate intake $(\beta=$ 0.016, p < 0.001) [Ruottinen et al., 2009] (LOE Ib).

In an intervention study by Anderson et al. [1992] (LOE Ib), a higher carbohydrate intake did not have a significant influence on the triglyceride concentration. In an intervention study by Kasim et al. [1993] (LOE Ib), carbohydrate intake increased by 11 EN\% (and at the same time dietary fibre intake increased by $4 \mathrm{~g}$ /day) did not influence the triglyceride concentration. In a study by Sacks et al. [1986] (LOE Ic), the high-carbohydrate diet (62 EN\%) did not influence the triglyceride concentration. An intervention study by Vartiainen et al. [1986] (LOE Ic) showed no influence of high-carbohydrate diet (60 EN\%) on the triglyceride concentration. A diet rich in carbohydrates and starch did not influence the triglyceride concentration in the intervention study by Kasim-Karakas et al. [2000] (LOE Ib). In an intervention study with 4 groups consisting of about 45 healthy subjects each and 54 EN\%, 39 EN\%, and $26 \mathrm{EN} \%$ carbohydrates, respectively (the latter with different proportions of saturated fatty acids), triglyceride concentrations were lowered in the periods with low carbohydrate intake, but this was only significant with the 26 EN\%-carbohydrate diet and low content of saturated fatty acids. After weight reduction, plasma triglyceride concentrations were slightly lower in all diet groups, but this was not significant [Krauss et al., 2006] (LOE Ib).

Particularly based on the results of the meta-analyses, the evidence regarding an increasing effect of an increased carbohydrate proportion (independent of the quality of the fatty acids in the diet) on the plasma triglyceride concentration is judged as convincing.

\subsection{Mono- and Disaccharides}

\section{Total and LDL Cholesterol}

In the Multiple Risk Factor Intervention Trial including 6,438 overweight men, energy intake was restricted in the intervention group. Therefore, only the findings in the control group at baseline and during the 6-year observation period could be used for analysis. In this group, differences in sucrose intake did not influence the plasma levels of total or LDL cholesterol. There was a significant inverse association between other mono- and disaccharides (naturally occurring sucrose, fructose, maltose and lactose in vegetables, fruit, wine or beer) and the total and LDL cholesterol concentrations [Tillotson et al., 1997a] (LOE IIb).

In a prospective cohort study over 4 years including 519 children at the age of 3-4 years, no association was found between the quantity of sucrose intake and the plasma total cholesterol concentration [Williams and Strobino, 2008] (LOE IIb).

In a 10 -week intervention study including 32 overweight men and women, plasma levels of total and LDL cholesterol significantly increased under the influence of fructose-sweetened beverages in the quantity of $25 \mathrm{EN} \%$ (percentage change: $+10.1, \mathrm{p}<0.0001$ and $+13.9, \mathrm{p}<0.01$ ) [Stanhope et al., 2009] (LOE Ib). In an intervention study (41 EN\% carbohydrates), isocaloric exchange of sucrose ( $25 \%$ of carbohydrates) for glucose syrup in 18 men over 1 year resulted in a significant reduction in the total cholesterol concentration, except in men who gained weight $(\mathrm{n}=5$; men without significant weight difference from 6.24 to $5.80 \mathrm{mmol} / \mathrm{l}$, men with significant weight reduction from 6.11 to $5.49 \mathrm{mmol} / \mathrm{l}, \mathrm{p}<0.025$ each) [Lock et al., 1980] (LOE Ib).

In an intervention study on sugar intake including 72 women over a period of 22 months, sucrose, fructose and xylitol did not differ regarding their effect on the total cholesterol concentration. After 22 months, the total cholesterol concentrations did not differ from baseline levels. Even the consumption of $>100 \mathrm{~g}$ fructose or xylitol per day did not lead to changes in the cholesterol concentration [Huttunen et al., 1976] (LOE Ib). In a 10-week nonrandomised and non-controlled intervention study in 7 overweight women, the replacement of $25 \mathrm{EN} \%$ of complex carbohydrates by fructose-sweetened beverages did not change the LDL cholesterol concentration [Swarbrick et al., 2008] (LOE Ic). In an intervention study including 644 children over several years, the association between carbohydrate intake and serum lipids as well as in dependence of the apoE phenotype was investigated. After adjustment, there was no association between total and LDL 
cholesterol concentrations and the intake of sucrose and fructose [Ruottinen et al., 2009] (LOE Ib).

Due to inconsistent study results, there is insufficient evidence regarding the influence of increased intake of mono- or disaccharides on the plasma levels of total and/ or LDL cholesterol.

\section{HDL Cholesterol}

In the CARDIA study, there was a significant inverse correlation in 4,734 white and black men and women between sucrose intake and plasma HDL cholesterol concentrations in the course of 7 years. Within this period, the BMI increased by an average of 2 units [Archer et al., 1998] (LOE IIb). In an analysis of the Multiple Risk Factor Intervention Trial including 6,438 overweight men, there was a significant inverse association between the quantity of sugar intake and the plasma HDL cholesterol concentration, and a significant positive association between the quantity of the intake of other mono- and disaccharides and the plasma HDL cholesterol concentration [Tillotson et al., 1997a] (LOE IIb). In the cohort study by Williams and Strobino [2008] (LOE IIb), at baseline there was no association found in 3-to 4-year-olds between the quantity of sugar intake and the HDL cholesterol concentration. At the end of the follow-up, a significant inverse association was found $(\mathrm{p}<0.05)$.

In an intervention study including 644 children over several years, the association between carbohydrate intake and serum lipids was investigated. Plasma HDL concentration decreased with increasing sucrose and fructose intake (significant for fructose: $\beta=-0.0065, p=0.02$ ) [Ruottinen et al., 2009] (LOE Ib). In a 10-week intervention study with 32 overweight men and women, the plasma HDL cholesterol concentration significantly increased under the influence of fructose-sweetened beverages in the amount of $25 \mathrm{EN} \%$ (percentage change: +3.5 , $\mathrm{p}<0.01$ ), under the influence of glucose-sweetened beverages it decreased significantly (percentage change: -2.4 , $\mathrm{p}<0.05$ ) [Stanhope et al., 2009] (LOE Ib).

In a 10-week non-randomised and non-controlled intervention study in 7 overweight women, the replacement of $25 \mathrm{EN} \%$ of complex carbohydrates by fructose-sweetened beverages did not change the HDL cholesterol concentration [Swarbrick et al., 2008] (LOE Ic).

Due to inconsistent study results, there is insufficient evidence regarding the influence of increased intake of mono- or disaccharides on the plasma levels of HDL cholesterol.

Evidence-Based Guideline of the German Nutrition Society

\section{Triglycerides}

In an analysis of the Multiple Risk Factor Intervention Trial including 6,438 overweight men, there was no association between the quantity of sugar intake or other mono- and disaccharides, respectively, and the plasma triglyceride concentration [Tillotson et al., 1997a] (LOE IIb). In the cohort study by Williams and Strobino [2008] (LOE IIb), no association was found between the quantity of sugar intake and the plasma triglyceride concentration.

According to a meta-analysis of 14 intervention studies over a period of 2-26 weeks with a total of 713 men and women, there is no significant association between an intake of $<100 \mathrm{~g}$ fructose/day and the plasma fasting triglyceride concentration and no significant influence of an intake of $<50 \mathrm{~g}$ fructose/day on the postprandial triglyceride concentration. With higher fructose quantities up to $350 \mathrm{~g} /$ day, the fasting plasma triglyceride concentrations significantly increase $(\mathrm{p}=0.05$ and $\mathrm{p}=0.001$, respectively) [Livesey and Taylor, 2008] (LOE Ia).

In addition to the intervention studies that have been included in this meta-analysis, further intervention studies are available. In a 10-week non-randomised and noncontrolled intervention study in 7 overweight women, the replacement of $25 \mathrm{EN} \%$ of complex carbohydrates by fructose-sweetened beverages did not result in an increase in the fasting triglyceride concentration, however, it led to a significant increase in the postprandial triglyceride concentration (from 5.1 to $12.3 \mathrm{mmol} / \mathrm{l}, \mathrm{p}=0.04$ ) [Swarbrick et al., 2008] (LOE Ic). In an intervention study including 644 children over several years, triglyceride concentrations increased significantly with increasing sucrose intake $(\beta=0.0011, \mathrm{p}<0.001)$ [Ruottinen et al., 2009] (LOE Ib). In a 10-week intervention study including 32 overweight men and women, the influence of glucose-sweetened beverages was compared with the influence of fructose-sweetened beverages in the quantity of $25 \% \mathrm{EN}$ each. The fasting triglyceride concentration rose significantly after consumption of the glucose-sweetened beverage $(+9.7 \mathrm{mg} / \mathrm{dl}, \mathrm{p}<0.05)$, while the fructose-sweetened beverage did not have any influence. In contrast, the postprandial triglyceride concentration increased significantly after consumption of the fructose-sweetened beverage (percentage change $+38.1 \mathrm{mg} / \mathrm{dl}, \mathrm{p}<0.0001$ ) [Stanhope et al., 2009] (LOE Ib).

In a 1-year intervention study, the replacement of sucrose intake by glucose syrup in 18 men resulted in a significant increase in the plasma triglyceride concentration (from 1.33 to $1.51 \mathrm{mmol} / \mathrm{l}, \mathrm{p}<0.05$ ) only in men who gained weight $(\mathrm{n}=5)$ [Lock et al., 1980] (LOE Ib).

Ann Nutr Metab 2012;60(suppl 1):1-58 
Due to the results of a meta-analysis, the evidence is judged as probable that there is no association between a fructose intake of $<100 \mathrm{~g} /$ day and the fasting plasma triglyceride concentration as well as between a fructose intake of $<50 \mathrm{~g} /$ day and the postprandial plasma triglyceride concentration, respectively. There is convincing evidence that with higher fructose intake of up to $350 \mathrm{~g} /$ day, the fasting plasma triglyceride concentration increases. The evidence regarding the effects of other mono- and disaccharides on plasma triglycerides is insufficient.

Because there are not enough studies available, the evidence regarding the influence of mono- and disaccharides on the plasma lipoprotein levels is insufficient, except for fructose. Fructose has a greater lipogenic effect and leads to a higher increase in the plasma triglyceride concentration than glucose and carbohydrates composed thereof [Tappy and Le, 2010]. However, fructose intake of $<100 \mathrm{~g} /$ day does not result in an increase in the fasting plasma triglyceride concentration and fructose intake of $<50 \mathrm{~g} /$ day does not lead to an increase in the postprandial plasma triglyceride concentration either.

\subsection{Polysaccharides}

Total and LDL Cholesterol

In the Multiple Risk Factor Intervention Trial, there was no significant association between the quantity of starch intake and the plasma levels of total as well as LDL cholesterol [Tillotson et al., 1997a] (LOE IIb). In an intervention study in children, the quantity of starch intake did not influence plasma levels of total as well as LDL cholesterol [Ruottinen et al., 2009] (LOE Ib).

Regarding the association between the intake of polysaccharides (starch) and plasma levels of total as well as LDL cholesterol, there is insufficient evidence due to the low number of study results.

\section{HDL Cholesterol}

The quantity of starch intake was not associated with the plasma LDL concentration in the study by Tillotson et al. [1997a] (LOE IIb). A diet rich in starch significantly lowered the HDL cholesterol concentration in the intervention study by Kasim-Karakas et al. [2000] (LOE Ic) $(\beta=-0.425, p<0.05)$.
There is insufficient evidence regarding the effects of polysaccharides (starch) on the plasma HDL cholesterol concentration due to the low number of study results.

\section{Triglycerides}

In the Multiple Risk Factor Intervention Trial, there was no significant association between the quantity of starch intake and the plasma triglyceride concentration [Tillotson et al., 1997a] (LOE IIb).

There is insufficient evidence regarding the effects of polysaccharides (starch) on the plasma triglyceride concentration due to the low number of study results.

\subsubsection{Dietary Fibre}

Total and LDL Cholesterol

In a prospective cohort study including 316 men and women over a period of 7 years, there was a significant inverse association between total and LDL cholesterol concentrations and the quantity of dietary fibre intake $(\beta=-1.387 \mathrm{mg} / \mathrm{dl}, \mathrm{p}<0.05)$ [de Castro et al., 2006] (LOE IIb). In a prospective cohort study over 4 years including 519 children at the age of 3-4 years, there was a significant inverse association determined between the quantity of dietary fibre intake and the plasma total cholesterol concentration $(\beta=-0.14, p<0.05)$ [Williams and Strobino, 2008] (LOE IIb).

In a 12 -year cohort study including 300 women, Millen et al. [2006] (LOE IIb) observed that higher carbohydrate (49 EN\%) and dietary fibre intake $(18 \mathrm{~g} / 1,000 \mathrm{kcal}) \mathrm{com}-$ pared with lower intake (38 EN\% and $9 \mathrm{~g} / 1,000 \mathrm{kcal}$ ) is not accompanied by lower total and LDL cholesterol concentrations. In another prospective cohort study including 2,909 subjects, plasma LDL cholesterol levels did not differ significantly after 10 years between white and black men and women with high dietary fibre intake $(10.5 \mathrm{~g} / \mathrm{MJ} ; 48$ EN\%) and those with low dietary fibre intake (5.9 g/MJ; 36 EN\% carbohydrates) [Ludwig et al., 1999] (LOE IIb). Another cohort study lasting 2.5 years and including 1,182 children at the age of 9 years showed no relation between dietary fibre intake and plasma levels of total cholesterol [Nicklas et al., 2002] (LOE IIb). In a prospective 18-months cohort study including 500 men and women, there was no association between the intake of total, water-soluble and insoluble dietary fibre or pectin and the plasma LDL cholesterol concentration [Wu et al., 2003] (LOE IIb).

A meta-analysis of 67 intervention studies with a mean study duration of 49 days and a total of 2,990 men and women showed that soluble dietary fibre (2-10 g/day) significantly lowers the plasma level of total cholesterol 
(-0.045 $\mathrm{mmol} / \mathrm{l} / \mathrm{g}$ soluble dietary fibre, $95 \%$ CI -0.054 ; $-0.035)$. Soluble dietary fibre also lowers the concentration of LDL cholesterol $(-0.057 \mathrm{mmol} / \mathrm{l} / \mathrm{g}, 95 \% \mathrm{CI}-0.070$; $-0.044)$. Daily intake of $3 \mathrm{~g}$ soluble dietary fibre from oats (about $80 \mathrm{~g}$ oatmeal) reduces plasma levels of total and LDL cholesterol by only $0.13 \mathrm{mmol} / \mathrm{l}$. As determined by several studies included into this meta-analysis, average daily quantities of oatmeal $(5.0 \mathrm{~g})$, psyllium $(9.1 \mathrm{~g})$, pectin $(4.7 \mathrm{~g})$ and guar $(17.5 \mathrm{~g})$ reduce the plasma levels of total and LDL cholesterol not very much, but significantly [Brown et al., 1999] (LOE Ia).

According to another meta-analysis of 8 intervention studies including 391 participants and a duration of 4-12 weeks, daily intake of 3-10 g $\beta$-glucan from barley results in a reduction in plasma levels of total $(-13.38 \mathrm{mg} / \mathrm{dl}, 95 \%$ CI $-18.46 ;-8.31)$ and LDL cholesterol (-10.02 mg/dl, $95 \%$ CI -14.03; -6.00) [Talati et al., 2009] (LOE Ia).

According to a Cochrane review including 10 controlled intervention studies with a duration of $4-8$ weeks, the consumption of whole-grain products ( 8 of them oatmeal-based) resulted in a significant decrease $(-0.19$ $\mathrm{mmol} / \mathrm{l}, \mathrm{p}=0.0005)$ in the plasma total cholesterol concentration compared with the control diet. The plasma concentration of LDL cholesterol, too, was significantly lower at the end of the studies than at baseline $(-0.18$ $\mathrm{mmol} / \mathrm{l}, \mathrm{p}<0.0001$ ) [Kelly et al., 2007] (LOE Ia).

In addition to the intervention studies included in the meta-analyses, further studies are available. In an intervention study including 53 men and women over 3 months, a high intake of $26 \mathrm{~g}$ dietary fibre/day ( $3.5 \mathrm{~g}$ soluble dietary fibre) and $55 \mathrm{EN} \%$ carbohydrates at constant body weight resulted in a decrease in the plasma concentrations of total (from 5.1 to $4.9 \mathrm{mmol} / \mathrm{l}, \mathrm{p}<0.05$ ) and LDL cholesterol (from 3.5 to $3.1 \mathrm{mmol} / \mathrm{l}, \mathrm{p}<0.05$ ), compared with a control group with $9 \mathrm{~g}$ dietary fibre/day (1.7 $\mathrm{g}$ soluble dietary fibre) and 45 EN\% carbohydrates [Aller et al., 2004] (LOE Ib). An increase in carbohydrate intake from 45 to $60 \mathrm{EN} \%$ and in dietary fibre intake from 18 to $35 \mathrm{~g} /$ day in the Family Heart Study including 287 men and women resulted in a significant reduction in the plasma total cholesterol concentration after 5 years $(-0.27 \mathrm{mmol} / \mathrm{l}, \mathrm{p}=0.008)$ [Connor et al., 1992] (LOE Ib). In the study of Sandström et al. [1992] (LOE Ib) including 30 men and women and lasting for 8 months, a diet rich in carbohydrates (58 EN\%) and dietary fibre ( $50 \mathrm{~g} /$ day) resulted in a significant decrease in the plasma total cholesterol concentration (women: from 4.21 to $3.8 \mathrm{mmol} / \mathrm{l}$; men: from 4.61 to $3.89 \mathrm{mmol} / \mathrm{l}$ ) compared to the control group (45 EN\% carbohydrates, $30 \mathrm{~g}$ dietary fibre/day). Body weight remained constant. The Multiple Risk Factor Intervention Trial showed a sig- nificant inverse association between dietary fibre intake and total and LDL cholesterol concentration in men ( $\mathrm{p}=$ 0.001 each) [Tillotson et al., 1997b] (LOE Ib). In a randomised controlled intervention study over 3 months including 67 men and women with hypercholesterolaemia, plasma total $(-0.23 \mathrm{mmol} / \mathrm{l}, \mathrm{p}=0.026)$ and LDL cholesterol concentrations $(-0.24 \mathrm{mmol} / \mathrm{l}, \mathrm{p}=0.0057)$ were lowered at unchanged body weight by soluble dietary fibre (13 g/day) [Tai et al., 1999] (LOE Ib).

In an intervention study including 110 men and women over 3 months, the addition of $11 \mathrm{~g}$ oats per day did not influence the plasma total and LDL cholesterol concentrations [Chen et al., 2006] (LOE Ib). An intervention study including 21 men and women over 18 months with a change from white bread to whole-grain bread consumption (increase from $13 \mathrm{~g}$ dietary fibre/day to $23 \mathrm{~g} /$ day; carbohydrate proportion stayed the same) at almost constant body weight did not result in a change of the plasma total cholesterol concentration [Eastwood et al., 1986] (LOE Ib). Change from white bread to whole-grain bread in 19 students over 19 weeks at constant body weight did not result in a changed total cholesterol concentration [Heaton et al., 1976] (LOE Ib). In the 1-year intervention study by Rock et al. [2004] (LOEK Ib), 393 women received a diet with an increased carbohydrate (increase from 57 to $65 \mathrm{EN} \%$ ) and dietary fibre intake (increase from 21 to $30 \mathrm{~g} /$ day). Body weight did not change significantly. This diet did not have any effect on the plasma levels of total and LDL cholesterol. In an intervention study including 644 children over 4 years, the association between carbohydrate and dietary fibre intake and serum lipids was investigated. Dietary fibre did not have a significant effect on plasma total and LDL cholesterol concentrations [Ruottinen et al., 2009] (LOE Ib).

In an intervention study with a duration of 6 months including 54 men and women, arabinogalactan ( $8.4 \mathrm{~g} /$ day) did not influence total and LDL cholesterol concentrations in comparison to a placebo [Marett et al., 2004] (LOE Ib).

According to the results of a Cochrane Review, there is convincing evidence that an increased consumption of whole-grain products reduces the plasma levels of total and LDL cholesterol. Regarding the effect of dietary fibre on the plasma concentrations of total and LDL cholesterol in total, there is possible evidence for an inverse risk relation. There is convincing evidence that an increase in the intake of total soluble dietary fibre lowers the plasma concentrations of total and LDL cholesterol. According to special studies, there is also convincing evidence that this also applies for oatmeal, psyllium, pectin and guar alone. 
There is convincing evidence that $\beta$-glucan from barley lowers the plasma concentrations of total and LDL cholesterol.

\section{HDL Cholesterol}

In the cohort study by Ludwig et al. [1999] (LOE IIb), after 10 years white men and women with a high dietary fibre intake had a higher plasma HDL cholesterol concentration than those with low dietary fibre intake. In black men and women, different quantities of dietary fibre intake did not influence the plasma HDL cholesterol concentration. In a prospective cohort study including 500 men and women over 18 months, the intake of total ( $\mathrm{r}=$ $0.091, \mathrm{p}=0.03)$ and soluble dietary fibre $(\mathrm{r}=0.114, \mathrm{p}=$ $0.01)$ and of pectin $(r=0.089, \mathrm{p}=0.04)$ was significantly positively associated with the HDL cholesterol concentration [Wu et al., 2003] (LOE IIb).

In the cohort study by Nicklas et al. [2002] (LOE IIb), an increased dietary fibre intake was not associated with changes of the plasma HDL cholesterol concentration. In the cohort study by Tillotson et al. [1997b] (LOE IIb), no association was shown between the intake of total as well as of soluble and insoluble dietary fibre and the HDL cholesterol concentration. In a cohort study with 300 women over 12 years, Millen et al. [2006] (LOE IIb) observed that a higher carbohydrate (49 EN\%) and dietary fibre intake (18 g/1,000 kcal) compared with a lower intake (38 EN\% and $9 \mathrm{~g} / 1,000 \mathrm{kcal}$ ) was not associated with a significantly lower plasma HDL cholesterol concentration. In the prospective cohort study by Williams and Strobino [2008] (LOE IIb) including 519 children at the age of 3-4 years, no association was found between the quantity of dietary fibre intake and the plasma HDL cholesterol concentration.

A meta-analysis of 67 controlled intervention studies with a mean study duration of 49 days and a total of 2,990 men and women showed that soluble dietary fibre in total significantly lowers the plasma level of HDL cholesterol to a small extent $(-0.002 \mathrm{mmol} / \mathrm{l} / \mathrm{g}, 95 \%$ CI -0.004 ; 0.0003). Detailed investigations showed that psyllium and guar, but not oatmeal and pectin, lower the plasma concentration of HDL cholesterol very slightly, but significantly [Brown et al., 1999] (LOE Ia).

According to another meta-analysis of 8 intervention studies with 391 participants and a duration of 4-12 weeks, daily intake of 3-10 g $\beta$-glucan from barley does not lower the concentration of plasma HDL cholesterol significantly $(p=0.07)$ [Talati et al., 2009] (LOE Ia).

According to a Cochrane review including 10 controlled intervention studies with a duration of $4-8$ weeks, the consumption of whole-grain products (8 of them oatmeal-based) did not result in a significant difference in the plasma HDL cholesterol concentration compared with the control diet [Kelly et al., 2007] (LOE Ia).

One of the studies that were not included into the meta-analyses [Aller et al., 2004] (LOE Ib) showed that a total daily intake of $25 \mathrm{~g}$ dietary fibre (including $3.5 \mathrm{~g}$ soluble dietary fibre) did not influence the plasma concentration of HDL cholesterol. In the intervention study of Chen et al. [2006] (LOE Ib), the addition of $11 \mathrm{~g}$ oats per day did not influence HDL cholesterol. In an intervention study including 644 children over 4 years, there was no significant association between the quantity of dietary fibre intake and the plasma HDL cholesterol concentration [Ruottinen et al., 2009] (LOE Ib). In a randomised controlled intervention study lasting for 3 months and including 67 men and women with hypercholesterolaemia, the plasma concentration of HDL cholesterol was significantly increased by an increased intake of soluble dietary fibre (13 g/day) compared with baseline (0.09 $\mathrm{mmol} / \mathrm{l}, \mathrm{p}<0.01)$. However, in the placebo group it was significantly increased as well $(0.1 \mathrm{mmol} / \mathrm{l}, \mathrm{p}<0.01)$ [Tai et al., 1999] (LOE Ib). There were no significant differences between the 2 groups. The authors assumed that a change of lifestyle factors could be the cause in both groups.

Sandström et al. [1992] (LOE Ib) demonstrated that a diet rich in carbohydrates (58 EN\%) and dietary fibre (50 $\mathrm{g} /$ day) over the course of 8 months in 30 men and women resulted in a significant decrease in the plasma HDL cholesterol concentration. In the intervention study by Rock et al. [2004] (LOE Ib), an increased carbohydrate (from 57 to $65 \mathrm{EN} \%$ ) and dietary fibre intake (from 21 to $30 \mathrm{~g} /$ day) led to a significant decrease in the plasma HDL cholesterol concentration.

There is convincing evidence that there is no association between the consumption of whole-grain products and the plasma HDL cholesterol concentration. There is probable evidence that dietary fibre in total does not influence the plasma HDL cholesterol concentration. According to the meta-analyses' results, there is convincing evidence that increasing the intake of soluble dietary fibre in total slightly lowers the plasma concentration of $H D L$ cholesterol. Special investigations showed that there is probable evidence that psyllium and guar lower the plasma concentration of HDL cholesterol and probable evidence that, in contrast, oatmeal and pectin do not. With convincing evidence, $\beta$-glucan from barley does not reduce the plasma concentration of HDL cholesterol. 
Triglycerides

In the cohort study by Ludwig et al. [1999] (LOE IIb), after 10 years white men and women with high dietary fibre intake had a lower plasma triglyceride concentration than subjects with low dietary fibre intake (from 88.5 to $80.5 \mathrm{mg} / \mathrm{dl}, \mathrm{p}=0.05)$. In black men and women, different quantities of dietary fibre intake did not significantly influence the plasma triglyceride concentration.

The cohort study by Tillotson et al. [1997b] (LOE IIb) did not show an association between the intake of total as well as of soluble and insoluble dietary fibre and plasma triglycerides. In the prospective cohort study by Williams and Strobino [2008] (LOE IIb) including 519 children at the age of 3-4 years, no association was found between the quantity of dietary fibre intake and the plasma triglyceride concentration. In the prospective cohort study by Wu et al. [2003] (LOE IIb), there was no significant association between the intake of total, soluble and insoluble dietary fibre as well as of pectin and plasma triglyceride concentrations.

A meta-analysis of 67 intervention studies with a mean study duration of 49 days and a total of 2,990 men and women showed that increased intake of soluble dietary fibre in total does not lower the plasma level of triglycerides. This also applies to special investigations with oatmeal, psyllium, pectin and guar [Brown et al., 1999] (LOE Ia).

According to another meta-analysis of 8 intervention studies with 391 participants and a duration of 4-12 weeks, daily intake of 3-10 g $\beta$-glucan from barley resulted in a significant reduction in the plasma triglyceride concentration $(-11.83 \mathrm{mg} / \mathrm{dl}, 95 \% \mathrm{CI}-20.12 ;-3.55)$ [Talati et al., 2009] (LOE Ia).

According to a Cochrane review including 10 controlled intervention studies with a duration of 4-8 weeks, the consumption of whole-grain products ( 8 of them oatmeal-based) did not result in a significant difference in the plasma triglyceride concentration compared with the control diet [Kelly et al., 2007] (LOE Ia).

In another intervention study by Aller et al. [2004] (LOE Ib), a total daily intake of $25 \mathrm{~g}$ dietary fibre (including $3.5 \mathrm{~g}$ soluble dietary fibre) did not influence the plasma triglyceride concentration. In the intervention study of Chen et al. [2006] (LOE Ib), the addition of $11 \mathrm{~g}$ oats per day did not influence the plasma triglyceride concentration [Chen et al., 2006] (LOE Ib). An intervention study including 21 men and women over 18 months with a change from white bread (14 g dietary fibre/day) to whole-grain bread consumption (23 g dietary fibre/day) did not result in a change of the plasma triglyceride con- centration [Eastwood et al., 1986] (LOE Ib). Change from white bread to whole-grain bread in 19 students over 19 weeks did not result in a changed triglyceride concentration [Heaton et al., 1976] (LOE Ib). Sandström et al. [1992] (LOE Ib) demonstrated that a diet rich in carbohydrates (58 EN\%) and dietary fibre (50 g/day) over the course of 8 months in 30 men and women did not influence the plasma triglycerides. In an intervention study including 644 children over several years, there was no significant association between the quantity of dietary fibre intake and the plasma triglyceride concentration [Ruottinen et al., 2009] (LOE Ib). In the study by Rock et al. [2004] (LOE Ib), an increased carbohydrate and dietary fibre intake led to a significant increase in the triglyceride concentration (from 1.04 to $1.17 \mathrm{mmol} / \mathrm{l}, \mathrm{p}<$ $0.05)$. In a randomised and controlled intervention study over 3 months including 67 men and women with hypercholesterolaemia, soluble dietary fibre (13 g/day) did not influence the plasma triglycerides [Tai et al., 1999] (LOE Ib).

There is convincing evidence that there is no association between the consumption of whole-grain products and plasma triglyceride concentrations. There is convincing evidence that there is no influence of dietary fibre in total on the plasma triglyceride concentration. According to the meta-analyses' results, there is convincing evidence that increasing the intake of soluble dietary fibre in total does not influence the plasma concentration of triglycerides. This also applies to special investigations with oatmeal, psyllium, pectin and guar. According to 1 meta-analysis, there is convincing evidence that $\beta$-glucan from barley reduces the plasma concentration of triglycerides.

\subsection{Glycaemic Index and Glycaemic Load}

Total and LDL Cholesterol

Ma et al. [2006] (LOE IIb) found in a longitudinal analysis of the data of a 1-year cohort study including 574 men and women, that there was a significant positive association between GL, but not GI, and plasma concentrations of total and LDL cholesterol. In the cross-sectional analysis, GL was significantly associated with lower total and LDL cholesterol concentrations. Another cohort study including 335 men and women with an observation period of 6 years showed a positive association between GI and total cholesterol concentration, but not with LDL cholesterol concentration in men. In men, GL was only 
significantly associated with the total cholesterol concentration in the age group of about 35 years; the association with the LDL cholesterol concentration was significant in all age groups. In women, there was no significant association between GI and the total cholesterol concentration, but there was a significant positive association regarding the LDL cholesterol concentration, however, only in the age group of about 35 years [Oxlund et al., 2006] (LOE IIb).

According to a Cochrane review including 13 intervention studies, a low-GI diet compared with a high-GI diet was accompanied by a lower $(-0.17 \mathrm{mmol} / \mathrm{l}, \mathrm{p}=0.03)$ total cholesterol concentration. The authors point out that a significant effect was only demonstrated in 1 of the 13 studies. Regarding LDL cholesterol, there was no significant difference [Kelly et al., 2004] (LOE Ia).

Another Cochrane review including 3 intervention studies with a duration of 5 weeks to 6 months showed that diets with low GI are accompanied by a significantly $(\mathrm{p}<0.05)$ greater reduction in plasma total and LDL cholesterol concentrations than diets with high GI [Thomas et al., 2007] (LOE Ia).

In the intervention study by Sichieri et al. [2007] (LOE Ib) including 203 women over 18 months, diets rich in carbohydrates (60 EN\% with 44 or 36 g dietary fibre/day, respectively) with high GI or with low GI did not have different effects on the total and LDL cholesterol concentrations. In a more recent intervention study over 6 months including 38 men in 2 groups with low GI or high GI, respectively, and simultaneous instructions to reduce weight by $2-3 \mathrm{~kg}$, a low GI was associated with lower total $(-0.45 \mathrm{mmol} / \mathrm{l}, \mathrm{p}<0.05)$ and LDL cholesterol concentrations $(-0.22 \mathrm{mmol} / \mathrm{l}, \mathrm{p}<0.05)$ than a high GI [Philippou et al., 2009] (LOE Ib).

There is probable evidence that a low GI is associated with a lower plasma concentration of total cholesterol. The evidence regarding the association between GI and the plasma LDL cholesterol concentration is insufficient due to inconsistent results. The evidence regarding the association between GL and the plasma concentrations of total and LDL cholesterol is insufficient due to the low number of studies.

\section{HDL Cholesterol}

In the cohort study by Ma et al. [2006] (LOE IIb), GI and GL, respectively, were not associated with the plasma HDL cholesterol concentration in the longitudinal analysis, however, in the cross-sectional analysis there was a significant inverse association with the HDL cholesterol concentration for both GI and GL. In the cohort study by Oxlund et al. [2006] (LOE IIb), no association was shown between GI or GL and the plasma HDL cholesterol concentration.

According to a Cochrane review including 13 intervention studies, there was no significant difference between a low-GI diet compared with a high-GI diet regarding the plasma HDL cholesterol concentration [Kelly et al., 2004] (LOE Ia).

According to another Cochrane review [Thomas et al., 2007] (LOE Ia), 3 intervention studies on the HDL cholesterol concentration showed quite heterogeneous results. In 1 of the studies, a low-GI diet resulted in a significant increase in the plasma concentration of HDL cholesterol (+0.95 mmol/l, p < 0.05) [Sloth et al., 2004] (LOE Ib), the other 2 studies showed no significant association [Bouché et al., 2002; McMillen-Price et al., 2006] (LOE Ib).

In the intervention study by Sichieri et al. [2007] (LOE Ib), a high-GI diet and a low-GI diet did not have different effects on the plasma HDL cholesterol concentration. In a more recent intervention study over 6 months including 38 men in 2 groups with low GI or high GI, respectively, and simultaneous instructions to reduce weight by $2-3 \mathrm{~kg}$, GI did not influence the HDL cholesterol concentration.

There is convincing evidence that the GI value does not influence the plasma HDL concentration. The evidence regarding the association between GL and the plasma concentration of HDL cholesterol is insufficient due to the low number of studies.

\section{Triglycerides}

In the cohort study by Ma et al. [2006] (LOE IIb), GI or GL, respectively, were not associated with the plasma triglyceride concentration in either the longitudinal or the cross-sectional analysis. The cohort study by Oxlund et al. [2006] (LOE IIb) showed no association between GI or GL and the plasma triglyceride concentration.

According to the result of a Cochrane review including 13 intervention studies, the GI of the diet does not influence the plasma triglyceride concentration [Kelly et al., 2004] (LOE Ia).

According to a meta-analysis including 30 intervention studies investigating different study collectives (men and women, healthy people, diabetics, patients at risk of coronary heart disease) and a total of about 800 subjects per test group (GL higher or lower), a lowered GL is associated with a significant reduction in the fasting tri- 
glyceride concentration after adjustment for fat intake. There was a decrease in the fasting triglyceride concentration by $>0.10 \mathrm{mmol} / \mathrm{l}$ per unit GL decrease $(\mathrm{p}<0.02)$ [Livesey et al., 2008] (LOE Ia). The heterogeneity of the individual results was particularly related to the differences between normal weight and overweight persons. In contrast to the studies with overweight persons (BMI $>25$ ), in the studies with normal-weight individuals (7 studies) there was a significant association regarding a lowered plasma fasting triglyceride concentration in case of reduced GL ( $\mathrm{p}<0.02)$.

The results of 4 intervention studies included into another Cochrane review [Thomas et al., 2007] (LOE Ia) were inconsistent. In 1 of the studies, a low-GI diet resulted in a significant decrease in the plasma triglyceride level [Ebbeling et al., 2005] (LOE Ib), the 3 others did not show a significant effect [Bouché et al., 2002; McMillenPrice et al., 2006; Sloth et al., 2004] (LOE Ib).

In an intervention study by Sichieri et al. [2007] (LOE Ib), low-GI diet and high-GI diet did not have a different effect on plasma triglyceride levels. In a more recent intervention study over 6 months including 38 men in 2 groups with low GI or high GI, respectively, and simultaneous instructions to reduce weight by $2-3 \mathrm{~kg}$, a low GI resulted in a reduction in the plasma triglyceride level after 6 months $(-0.39 \mathrm{mmol} / \mathrm{l}, \mathrm{p}<0.05)$ [Philippou et al., 2009] (LOE Ib).

There is probable evidence that a high GI has no influence on the plasma fasting triglyceride concentration. According to the results of a meta-analysis, there is probable evidence that a high GL leads to an increased plasma fasting triglyceride concentration in normal-weight persons.

\subsection{Need for Research regarding Dyslipoproteinaemia}

In many studies, a change in carbohydrate intake is associated with a change in dietary fibre intake. Randomised controlled long-term intervention studies are urgently needed to investigate the influence of a change in carbohydrate intake (also regarding different carbohydrates) and of soluble and insoluble dietary fibre intake independently of each other.

Changing to a diet rich in carbohydrates and low in fat is often associated with spontaneous weight loss because of the lower energy density of this diet (DGE 2006). Due to the changed energy balance, the effects of food ingredients on the plasma lipoproteins are modified as well.
Additional controlled intervention studies regarding the effects of high-carbohydrate diets (including glucose versus fructose) on plasma lipoproteins at reliably constant body weight have to be performed. Extent and duration of carbohydrate-induced hypertriglyceridaemia in healthy individuals depending on the type and quantity of the ingested carbohydrates have to be investigated systematically.

Insufficient data are available regarding the effects of mono- and disaccharides as well as polysaccharides (starch) on plasma lipoproteins. Additional long-term randomised controlled intervention studies are necessary.

\section{Quantity and Quality of Carbohydrate Intake and Primary Prevention of Hypertension}

The data base for analyzing the relation between carbohydrate intake and the risk of hypertension compared with other diseases considered in this guideline is characterised by the low number of studies that can be used for evidence judgement.

\subsection{Proportion of Carbohydrates}

In the analyses of the cohort studies, carbohydrate intake (as relative proportion of energy intake) was not associated with blood pressure [Alonso et al., 2006; Ludwig et al., 1999; Stamler et al., 2002; Stamler et al., 1997] (all LOE IIb). In an intervention study with 662 girls and boys and the aim to change the lipoprotein composition by specifically modifying nutrition over 3 years, there was no change of blood pressure that was associated with modified carbohydrate intake [Simons-Morton et al., 1997] (LOE Ib). The results of 10 crossover intervention studies over 3-14 weeks, in which the intake of carbohydrates was increased isoenergetically at the expense of monounsaturated fatty acids, were summarised in a meta-analysis [Shah et al., 2007] (LOE Ia). The central finding of this meta-analysis was that increasing the intake of carbohydrates in exchange of monounsaturated fatty acids resulted in higher blood pressure (systolic blood pressure $\mathrm{p}=0.02$; diastolic blood pressure $\mathrm{p}=0.05$ ). However, the studies were very heterogeneous regarding this finding, which may be due to the different kinds of carbohydrates used for the intervention, according to the authors. 
There is possible evidence that there is no association between the proportion of nutritional carbohydrates and elevated blood pressure. The results of the cohort studies and the intervention study in children are consistent regarding a lack of an association; however, there was an increased risk in the meta-analysis of the intervention studies in adults with replacement of fats by carbohydrates. Therefore, the finding that there is no association should be considered with caution. Furthermore, there is possible evidence that the replacement of monounsaturated fatty acids by carbohydrates results in a short-term increase in blood pressure.

\subsection{Mono- and Disaccharides}

An analysis of the Nurses' Health Study I, the Nurses' Health Study II and the Health Professionals Follow-UP Study did not find evidence that the intake of fructose is associated with the development of hypertension [Forman et al., 2009] (LOE IIb). The intake of sucrose was not associated with blood pressure in the MRFIT Study [Stamler et al., 1997] (LOE IIb). During a 10-week intervention with either sucrose or artificial sweeteners including 41 adults $(n=21$ in the sucrose group, $n=20$ in the artificial sweeteners group) and the intake of other carbohydrates remaining the same, an increase in sucrose intake (from 11 to $27 \mathrm{EN} \%$ ) was associated with higher blood pressure and weight gain (systolic blood pressure: $3.8 \mathrm{~mm} \mathrm{Hg}$; diastolic blood pressure: $4.1 \mathrm{~mm}$ $\mathrm{Hg}$ ). The intake of foods sweetened with artificial sweeteners resulted in weight reduction as well as a lowered blood pressure [Raben et al., 2002] (LOE Ib). The subsequent multivariate analysis indicated that the increase in blood pressure was not only due to the change in body weight, but also was directly associated with the intake of sucrose. The analysis of the Framingham Heart Study did not reveal any evidence that sugar-sweetened beverages influence blood pressure [Dhingra et al., 2007] (LOE IIb).

There is possible evidence that there is no association between long-term intake of fructose or sucrose and blood pressure increase. Furthermore, there is possible evidence that there is no association between the intake of sugar-sweetened beverages and the risk of hypertension. This judgement is based on 1 cohort study only, but is confirmed by the judgement of the individual components of sweetened beverages like fructose and sucrose.

\subsection{Polysaccharides}

In the MRFIT Study, a positive association was observed over 6 years between the intake of starch and blood pressure levels (systolic blood pressure $\mathrm{p}<0.001$; diastolic blood pressure $\mathrm{p}<0.001$ ) [Stamler et al., 1997] (LOE IIb). On the other hand, no association was observed in cohort studies between the consumption of refined grain products and blood pressure levels [Steffen et al., 2005; Wang et al., 2007] (both LOE IIb), except in the study by Sahyoun et al. [2006] (LOE IIb) with 535 participants which showed a direct positive association between the intake of refined grain products and the level of systolic blood pressure $(\mathrm{p}=0.05)$.

The available data on the intake of polysaccharides or refined grain products, respectively, and blood pressure levels comprise only a few studies and are inconsistent; thus, the strength of the evidence is insufficient.

\subsubsection{Dietary Fibre}

In some cohort studies, the association between dietary fibre intake and the development of the blood pressure level or the risk of hypertension, respectively, was investigated. Mostly, inverse associations between dietary fibre intake and blood pressure levels have been observed [Alonso et al., 2006; Ascherio et al., 1996; Ascherio et al., 1992; Ludwig et al., 1999; Stamler et al., 1992; Witteman et al., 1989] (all LOE IIb). Some of these associations were not observed any more if the clinical diagnosis of hypertension was investigated instead of the blood pressure development. In these studies, the sources of dietary fibre played only a minor role [Alonso et al., 2006; Ascherio et al., 1996; Ascherio et al., 1992] (all LOE IIb). In the CARDIA study by Ludwig et al. [1999] (LOE IIb), inverse associations were only shown in white participants (systolic blood pressure $\mathrm{p}=0.01$; diastolic blood pressure $\mathrm{p}<0.001$ ), not in black people. In a Danish intervention study with 18 men, simultaneous increase in dietary fibre intake and a reduction in fat intake over 8 months resulted in a lowering of systolic blood pressure (from 123 to $119 \mathrm{~mm} \mathrm{Hg}, \mathrm{p}=0.002$ ), but not of diastolic blood pressure [Sandström et al., 1992] (LOE Ib). This result was concordant with an Australian intervention study including 74 overweight adults over 16 weeks. Dietary fibre and protein content was increased by lupin kernel flour (systolic blood pressure $\mathrm{p}=0.03$ ) [Lee et al., 2009] (LOE Ib).

Similar conclusions like in studies with dietary fibre as exposure factor were drawn from studies that investi- 
gated whole-grain products. Except for 1 small cohort study with 535 participants [Sahyoun et al., 2006] (LOE IIb), in the other studies a reduced risk of hypertension was observed with an increasing consumption of wholegrain products [Flint et al., 2009; Steffen et al., 2005; Wang et al., 2007] (all LOE IIb). The analysis of an intervention study with 6- to 11-year-old children showed an inverse relation between the intake of dietary fibre and blood pressure levels [Simons-Morton et al., 1997] (LOE Ib). Two meta-analyses from 2004 and 2005 have summarised the effects of dietary fibre in randomised intervention studies, including many studies on individuals with different blood pressure levels [Streppel et al., 2005; Whelton et al., 2005] (both LOE Ia). The data basis of the 2 meta-analyses mostly was overlapping; both showed that an average increase in intake of $11 \mathrm{~g}$ dietary fibre/day lowered both systolic and diastolic blood pressure in people with hypertension. The findings in normotensive persons were different. While the meta-analysis by Streppel et al. [2005] showed a lowering of diastolic blood pressure values in normotensive persons, this relation was also seen in the meta-analysis by Whelton et al. [2005], but it was not significant. In an intervention study including 36 middle-aged men who additionally consumed $14 \mathrm{~g}$ dietary fibre/day either in the form of oats or in form of wheat over 12 weeks, no changes in blood pressure levels were shown [Davy et al., 2002] (LOE Ib).

Based on the results of the present studies, there is probable evidence that increased dietary fibre consumption in a population with different blood pressure levels lowers the risk of hypertension. This also applies to the food group of whole-grain products.

\subsection{Glycaemic Index and Glycaemic Load}

Similar to the carbohydrate proportion, GL as well was not associated with the risk of hypertension in a Spanish cohort study [Alonso et al., 2006] (LOE IIb). An intervention study in 38 persons showed that a low-GI diet resulted in a decrease in the 24-hour systolic blood pressure [Phillippou et al., 2009] (LOE Ib). In contrast, the intervention with a high-GI diet did not have an effect on blood pressure levels [Phillippou et al., 2009] (LOE Ib).

There are not enough data available to judge the relation between diets with different GI and GL values and the blood pressure level, thus, the evidence is judged as insufficient.

Evidence-Based Guideline of the German Nutrition Society

\subsection{Need for Research regarding Hypertension}

Because hypertension is a common finding in the general public, more analyses of the existing cohort studies in which this disease is an endpoint should be conducted. As the summary of the evidence shows, it still remains open if the intake of mono- and disaccharides does not increase blood pressure levels, as it was observed in most cohorts so far. Therefore, this question should be further addressed in controlled intervention studies and additional cohort studies should be analysed.

It will be important to be able to better understand the effects of dietary fibre and whole-grain products on blood pressure regulation. It is unclear whether the observed effects of a diet rich in dietary fibre are due to a change in body weight and distribution of body fat (visceral and subcutaneous fat) or whether they are caused by a changed insulin/glucose metabolism.

\section{Quantity and Quality of Carbohydrate Intake and Primary Prevention of the Metabolic Syndrome}

Within the search, a limited number of studies ( $n=7$; 6 prospective cohort studies and 1 meta-analysis) on the relation between carbohydrate intake and the metabolic syndrome were found. No intervention studies were found. The studies found were very heterogeneous and differed significantly with regard to the number of participants, the age of the subjects, the observation period, the country of investigation, and the ethnic origin of the participants. The majority of study participants were USAmericans [Carnethon et al., 2004; Dhingra et al., 2007; Lutsey et al., 2008; Nettleton et al., 2009; Ventura et al., 2006]. In the 2 other prospective studies, subjects from Iran [Mirmiran et al., 2008] and from Brazil [Damiao et al., 2006], respectively, were investigated.

In our search, only those studies were chosen that correspond to the definition of the metabolic syndrome according to the criteria of the NCEP Expert Panel [2001] or Alberti et al. [2005]. However, it has to be mentioned that most authors set the limit regarding fasting blood glucose level at $110 \mathrm{mg} / \mathrm{dl}$ (according to the definition of the NCEP Expert Panel 2001), while Dhingra et al. [2007] used the more recent threshold of $100 \mathrm{mg} / \mathrm{dl}$. Damiao et al. [2006] used ethnic-specifically modified thresholds regarding waist circumference or abdominal obesity, while Mirmiran et al. [2008] did not. The use of different levels may be the reason for non-consistent results. On the one hand, with the application of the lower levels re-

Ann Nutr Metab 2012;60(suppl 1):1-58 
garding fasting blood glucose, more people with metabolic syndrome might have been identified. On the other hand, some ethnic groups show metabolic syndrome already below the level of the NCEP ATP III for Caucasians, so they would have been identified as well if the ethnicspecific thresholds would have been applied.

\subsection{Proportion of Carbohydrates}

Three cohort studies published in 2001 and later were found that investigated the influence of the carbohydrate proportion on the risk of the metabolic syndrome.

In the Coronary Artery Risk Development in Young Adults (CARDIA) study including 4,192 men and women (49\% Afro-Americans) at the age of 18-30 years at baseline, a positive association between carbohydrate intake and the risk of the metabolic syndrome was observed after a mean follow-up time of 13.6 years. Compared with a carbohydrate intake of $25-41 \%$ of total energy intake (EN\%), the risk increased to $1.63(95 \% \mathrm{CI} 1.06 ; 2.51)$ with an intake of 52-85 EN\% [Carnethon et al., 2004] (LOE IIb).

A positive association was shown as well in an Iranian study including 410 men and women at the age of 18-74 years. After 3.5 years of follow-up time, the risk of developing the metabolic syndrome increased by 2.7 -fold in the highest quartile of carbohydrate intake (63-77 EN\%) compared with the reference quartile (33-54 EN\%) $(\mathrm{OR}=2.7$; 95\% CI 1.2; 6.5; p < 0.01) [Mirmiran et al., 2008] (LOE IIb).

Damiao et al. [2006] analysed data of a Japanese-Brazilian cohort with 151 participants aged $40-79$ years who were followed up over 7 years. The modified criteria regarding waist circumference (women: $\geq 80 \mathrm{~cm}$; men: $\geq 90 \mathrm{~cm}$ ) for Asians were used in the diagnosis. Neither in women nor in men there were any differences regarding absolute and relative carbohydrate intake between subjects with and without the metabolic syndrome [Damiao et al., 2006] (LOE IIb).

The evidence regarding an association between the dietary proportion of carbohydrates and the occurrence of the metabolic syndrome is judged as insufficient due to the low number of prospective studies.

\subsection{Mono- and Disaccharides}

Regarding an association between the intake of monoand disaccharides and the risk of the metabolic syndrome, no prospective study was found. Three cohort studies and 1 meta-analysis were found on the influence of sugar-sweetened beverages on the metabolic syndrome.

Over a period of 8 years, Ventura et al. [2006] observed 152 white girls who were 5 years old at baseline. A statistical procedure taking 6 risk factor variables of the metabolic syndrome into account was used to classify the girls into 4 groups at the age of 13 years: 'Low risk of metabolic syndrome', 'low risk of dyslipoproteinaemia', 'low risk of hypertension' and 'high risk of metabolic syndrome'. Regarding their diet, the groups only differed in the consumption of sugar-sweetened beverages. The group 'high risk of metabolic syndrome' consumed significantly more $(\mathrm{p}<0.001)$ portions of sugar-sweetened beverages than all other groups, which accounted for a difference of $1 / 2$ to $2 / 3$ portions of sugar-sweetened beverages per day over the observation period [Ventura et al., 2006] (LOE IIb).

The analysis of successive examination visits of the Framingham Heart Study with middle-aged men and women showed that daily consumption of 1 or more portions of sweetened beverages (energy-containing and free) compared with no consumption was associated with an increased risk of the metabolic syndrome after a mean follow-up time of 4 years ( $\mathrm{OR}=1.44,95 \%$ CI $1.2 ; 1.74)$. If only energy-containing sweetened beverages were included, the risk of the metabolic syndrome at daily consumption $\geq 1$ portion compared with occasional consumption ( $<1$ portion/week) showed only a marginal statistically significant increase $(\mathrm{OR}=1.62,95 \% \mathrm{CI} 0.96$; $2.75 ; \mathrm{p}=0.07$ ) [Dhingra et al., 2007] (LOE IIb).

In the Atherosclerosis Risk in Communities (ARIC) study including 9,514 men and women between 45 and 64 years of age at baseline, the consumption of sugar-sweetened beverages ( 1 portion/day) after 9 years of follow-up was only marginally significantly associated with a slightly increased risk of the metabolic syndrome, too $($ HR extreme tertiles $=1.09,95 \%$ CI $0.99 ; 1.19 ; \mathrm{p}$ trend $=$ 0.07) [Lutsey et al., 2008] (LOE IIb).

The results of the 2 latter studies as well as non-published results of the study by Nettleton et al. [2009] were included in a meta-analysis from 2010 , so that this analysis was based on the data of 19,431 participants $(5,803$ cases) [Malik et al., 2010] (LOE IIa). Comparing highest and lowest intake (between $<1$ portion/month and $\geq 1-2$ portions/day) revealed a significantly increased risk of the metabolic syndrome ( $\mathrm{RR}=1.20,95 \% \mathrm{CI} 1.02 ; 1.42)$. The studies were largely consistent, all of them showed a positive association. 
The evidence regarding an association between the consumption of sugar-sweetened beverages and the occurrence of the metabolic syndrome is judged as possible.

\subsection{Polysaccharides}

No study could be identified that matched the criteria for the literature search defined a priori.

\subsubsection{Dietary Fibre}

Three cohort studies were found that investigated the association between dietary fibre intake and the metabolic syndrome. The source of dietary fibre was not specified in these studies.

In the CARDIA study $(\mathrm{n}=4,192)$, the risk of the metabolic syndrome in the lowest quintile of dietary fibre intake (women: 0.3-2.7 g/day; men: 0.6-3.6 g/day) did not differ significantly from the risk in the highest quintile after multivariate adjustment (women: $6.9-33 \mathrm{~g} / \mathrm{day}$; men: 8.6-29.8 g/day) $(\mathrm{OR}=1.12,95 \%$ CI 0.86; 1.47) [Carnethon et al., 2004] (LOE IIb). In an Iranian cohort (n = 410), dietary fibre intake was not associated with the risk of the metabolic syndrome either $(\mathrm{OR}=1.5,95 \% \mathrm{CI} 0.5$; 4) [Mirmiran et al., 2008] (LOE IIb).

In a Japanese-Brazilian cohort analysed by Damiao et al. [2006] (LOE IIb), dietary fibre intake at baseline of men and women who had developed metabolic syndrome after 7 years of follow-up ( $\mathrm{n}=31$ and 26 , respectively) did not differ from the intake of men and women without the metabolic syndrome ( $\mathrm{n}=53$ and 41, respectively).

The present studies indicate with possible evidence that there is no association between dietary fibre intake and the occurrence of the metabolic syndrome.

One cohort study was found regarding the association between the consumption of whole-grain products or refined grain products and the metabolic syndrome.

In the Atherosclerosis Risk in Communities Study, no association was observed between the consumption of whole-grain products or refined grain products and the occurrence of the metabolic syndrome during an observation period of 9 years [Lutsey et al., 2008] (LOE IIb).

Because there is only 1 prospective study available, the evidence regarding an association between the consumption of whole-grain products or refined grain products and the metabolic syndrome is judged as insufficient.

Evidence-Based Guideline of the German Nutrition Society

\subsection{Glycaemic Index and Glycaemic Load}

No cohort studies on this aspect were found.

\subsection{Need for Research regarding Metabolic Syndrome}

The number of identified studies regarding all types of carbohydrates is too low and the results are too inconsistent to derive significant associations thereof with higher strength of the evidence. To achieve a more comprehensive investigation of the association between carbohydrate intake and prevention of the metabolic syndrome, there is an urgent need for additional prospective studies, ideally intervention studies as well. There are hardly any studies on the influence of nutrition during childhood and adolescence on the prevention of the metabolic syndrome, which would be of interest in this context, too.

\section{Quantity and Quality of Carbohydrate Intake and Primary Prevention of Coronary Heart Disease}

\subsection{Proportion of Carbohydrates}

In recent epidemiological studies, the association between carbohydrate intake and the risk of coronary heart disease (CHD) has most often been investigated indirectly. To determine the influence of fat and fatty acids on the risk of CHD, so-called food-exchange models have been used that allowed the isoenergetic exchange of energy intake from one source of energy (for example fat) to another one (for example carbohydrates). In a recently performed pooled analysis of data from 11 prospective cohort studies [Jakobsen et al., 2009] (LOE IIa), the question was investigated whether saturated fatty acids (SFA) in the diet should be replaced by monounsaturated fatty acids (MUFA), polyunsaturated fatty acids (PUFA) or carbohydrates to reduce the risk of CHD. During the 4-10 years of follow-up, 5,249 incident CHD cases occurred, 2,155 of which were fatal. The substitution of SFA with PUFA was associated with a significantly reduced risk of CHD. The substitution of SFA with MUFA did not influence the risk of CHD. With the replacement of $5 \mathrm{EN} \%$ from SFA by the same amount of energy from carbohydrates, there was no association with the occurrence of fatal CHD (RR $=0.96 ; 95 \%$ CI 0.82; 1.13), but a slight and statistically significant increase in the overall CHD risk $(\mathrm{RR}=1.07 ; 95 \% \mathrm{CI} 1.01 ; 1.14)$. No explanation was provided for the contradictory findings regarding fatal CHD 
and total CHD. The heterogeneity between the studies was not statistically significant. The authors concluded from this meta-analysis that in order to reduce the risk of CHD, SFA in the diet should be replaced by PUFA and not by MUFA or carbohydrates.

A recently published meta-analysis of intervention studies [Mozaffarian et al., 2010] also showed that an exchange of SFA by PUFA results in a significantly lowered CHD risk.

Another meta-analysis based on prospective cohort studies by Siri-Tarino et al. [2010] (LOE IIa) did not find a significant effect of the replacement of SFA by carbohydrates on the risk of CHD. However, in this analysis there was no significant association between SFA intake and the risk of CHD either.

There are some additional recent and older studies available that investigated the association between carbohydrate intake and the occurrence of CHD without a socalled food-exchange model. They are described in the following.

In a study including 4,546 North American women and men the association between carbohydrate intake and the risk of CHD was investigated by means of a nutrient-density model. According to the results of this study, an increasing dietary energy percentage from carbohydrates (per $1 \mathrm{EN} \%$ ) is associated with a significantly reduced CHD risk by $4 \%(\mathrm{RR}=0.96 ; 95 \%$ CI $0.94 ; 0.99)$ in the age group of $30-59$ years. However, in the age group of 60-79 years, no significant effect was observed [Esrey et al., 1996] (LOE IIb).

Similar results were obtained from another prospective cohort study, the Swedish Women's Lifestyle and Health Study [Lagiou et al., 2007] (LOE IIb). In this study, 42,237 women aged 30-49 years were recruited and followed up for 12 years. The results show that a reduced consumption of carbohydrates by 1 decile was associated with an increased risk of premature death by $6 \%$ (95\% CI 1.00; 1.12); a significant association with CHD mortality could not be detected (RR $=10 \%$; $95 \%$ CI $0.96 ; 1.26$ ).

An analysis of the Nurses' Health Study did not find a significant association between the carbohydrate proportion of the diet and the risk of CHD. In this analysis, the low-carbohydrate-diet score was used as exposure; a high score reflects low carbohydrate intake and high intake of fat and proteins. During the 20-year follow-up, 1,994 cases of CHD occurred in the cohort. After multivariate adjustment the relative risk (RR) of CHD in women in the 10 th decile of the score was $0.94(95 \%$ CI $0.76 ; 1.18)$ compared with those in the 1st decile. Thus, a diet low in carbohydrates and high in fat and proteins was not signifi- cantly associated with the risk of $\mathrm{CHD}$, whereas the RR for carbohydrate deciles taking fat and protein from vegetable sources into account was 0.70 (95\% CI 0.56; 0.88). Additionally, each macronutrient was examined separately; there was no significant association between carbohydrate intake and the risk of CHD in the multivariate model ( $\mathrm{RR}=1.22$; 95\% CI 0.95; 1.56) [Halton et al., 2006] (LOE IIb).

In the Honolulu Heart Study [McGee et al., 1984] (LOE IIb), more than 8,000 men of Japanese origin were examined in Hawaii. Men who developed CHD or died of it had a higher proportion of energy intake from protein, fat, SFA and PUFA than men who remained free of CHD $(p<0.01)$, therefore, the proportion of carbohydrates was lower in men who developed CHD (not significant). Without adjustment for energy intake, the intake of carbohydrates and starch was inversely associated with the risk of CHD in the multivariate model (significant for carbohydrates and fatal CHD as well as for starch and total CHD, p < 0.05). An earlier analysis of this study obtained similar results [Yano et al., 1978] (LOE IIb).

In a study from Puerto Rico [Garcia-Palmieri et al., 1980] (LOE IIb), 8,218 urban and rural Puerto Rican men were observed over 6 years. It was shown that in urban men a lower consumption of carbohydrates, particularly starch, was associated with an increased risk of CHD $(\mathrm{p}<0.01)$ and deaths from CHD $(\mathrm{p}<0.05)$. In the rural population, no significant effect was shown.

Gordon et al. [1981] (LOE IIb) analysed data from the Puerto Rico Heart Health Program, the Honolulu Heart Study and the Framingham Study. In the Framingham Study, there was no significant association between the intake of carbohydrates, starch and sugar and the risk of incident and fatal CHD. In contrast, the studies from Puerto Rico and Hawaii showed that the mean intake of starch was significantly lower in persons with CHD ( $<<$ $0.05)$ and CHD-related deaths ( $<<0.01)$. Additionally, the numbers of CHD cases and CHD-related deaths significantly increased with decreasing total carbohydrate intake ( $\mathrm{p}<0.01$ ) [Gordon et al., 1981] (LOE IIb).

In summary, only the pooled analysis by Jakobsen et al. [2009] found a positive association between carbohydrate intake with exchange for SFA and the overall risk of $\mathrm{CHD}$, but there was no association between carbohydrate intake and the risk of fatal CHD. In contrast, the metaanalysis by Siri-Tarino et al. [2010] and all other listed observational studies showed either no significant association or a significant inverse association between carbohydrate intake and risk of CHD. 
The aim of the intervention study Women's Health Initiative Dietary Modification Trial (WHI) [Howard et al., 2006] (LOE Ib) was, in addition to fat reduction, an increase in the intake of fruit, vegetables and cereal products. After 1 year of intervention, carbohydrate intake in the intervention group was $58.3 \%$ of energy intake (EN\%) and in the control group $48.0 \mathrm{EN} \%$; after 6 years, the difference was similar with 53.9 EN\% in the intervention group and $43.9 \mathrm{EN} \%$ in the control group. Overall, the intervention did not have a significant influence on the incidence of CHD. After exclusion of participants with prevalent cardiovascular disease, the RR of CHD in postmenopausal women was 0.94 (95\% CI 0.86; 1.02).

There is possible evidence that an increase in carbohydrate intake or in the proportion of carbohydrates to total energy intake, respectively, does not influence the risk of CHD. This judgement of the evidence is due to the contradictory data.

\subsection{Mono- and Disaccharides}

A prospective cohort study [Fung et al., 2009] (LOE IIb) examined differences in the influence of various sweetened beverages on the risk of CHD. The beverages were classified into 4 different groups: all sugar-sweetened beverages, colas, non-cola carbonated soft drinks as well as fruit nectars and drinks. After multivariate adjustment, a positive association was shown between the intake of sugar-sweetened beverages ( $\mathrm{RR}=1.28$; $95 \% \mathrm{CI} 1.14 ; 1.44)$, colas $(\mathrm{RR}=1.35 ; 95 \% \mathrm{CI} 1.15 ; 1.57)$ as well as fruit nectars and drinks $(\mathrm{RR}=1.33$; 95\% CI $1.03 ; 1.71)$ and the occurrence of CHD and CHD-related deaths. The intake of noncola carbonated soft drinks was not significantly associated with the risk of CHD [Fung et al., 2009] (LOE IIb).

Another cohort study [Liu et al., 2000b] (LOE IIb) examined the influence of the intake of sucrose, fructose and lactose on the risk of CHD. For this purpose, 75,521 women at the age of 38-63 years were observed over a period of 10 years. There was no significant association shown between the intake of mono- and disaccharides and the risk of CHD.

Due to the low number of studies, the evidence regarding the association between the intake of mono- and disaccharides or the consumption of sugar-sweetened beverages, respectively, and the risk of $C H D$ is judged as insufficient.

Evidence-Based Guideline of the German Nutrition Society

\subsection{Polysaccharides}

The influence of starch on the risk of CHD was analysed in 4 older cohort studies [McGee et al., 1984; Yano et al., 1978; Garcia-Palmieri et al., 1980; Gordon et al., 1981] (all LOE IIb) that have been described above in more detail in subparagraph 8.1.

In the Honolulu Heart Study [McGee et al., 1984] (LOE IIb), the intake of starch that was not adjusted for energy intake was significantly inversely associated with the risk of CHD. An earlier analysis of this study showed similar results [Yano et al., 1978] (LOE IIb). According to the results of a study in Puerto Rico [Garcia-Palmieri et al., 1980] (LOE IIb), in urban men a lower intake of starch was associated with an increased risk of CHD overall $(p<0.01)$ and CHD-related deaths $(p<0.05)$. In men of a rural population, no association was shown. In a combined analysis of data from the Puerto Rico Heart Health Program, the Honolulu Heart Study and the Framingham Study, there was no significant association in the Framingham Study between the intake of starch and the risk of fatal and non-fatal CHD. In contrast, the studies from Puerto Rico and Hawaii showed that the mean intake of starch was significantly lower in persons with CHD $(\mathrm{p}<$ $0.05)$ and CHD-related deaths $(\mathrm{p}<0.01)$ [Gordon et al., 1981] (LOE IIb).

Due to the low number of older studies with partially less than optimal statistical analysis, the evidence regarding the association between the intake of starch and the risk of CHD is judged as insufficient.

\subsubsection{Dietary Fibre}

Total Dietary Fibre

The association between dietary fibre intake and the risk of CHD was investigated in many observational studies which formed the basis of the available metaanalyses and the pooled analysis.

A pooled analysis of 10 prospective cohort studies with a follow-up period of 6-10 years comprised 5,249 incident CHD cases, 2,011 of which were fatal [Pereira et al., 2004] (LOE IIa). In the adjusted model with additional correction of measurement errors, the risk of CHD was lowered by $14 \%$ per increase of $10 \mathrm{~g} /$ day of total dietary fibre $(\mathrm{RR}=0.86 ; 95 \% \mathrm{CI} 0.78 ; 0.96)$, the risk of fatal CHD was reduced by $27 \%(\mathrm{RR}=0.73 ; 95 \% \mathrm{CI} 0.61$; 0.87).

A meta-analysis from 2005 including 7 studies with a total of more than 150,000 participants also showed a significant inverse association between dietary fibre intake

Ann Nutr Metab 2012;60(suppl 1):1-58 
and the risk of CHD $(\mathrm{RR}=0.71 ; 95 \%$ CI $0.47 ; 0.95)[\mathrm{An}$ derson, 2005] (LOE IIa).

In a more recent meta-analysis [Mente et al., 2009] (LOE IIa), 146 prospective cohort studies (125 studies on primary prevention) with a mean follow-up of 11 years were included. The analysis regarding the association between dietary fibre intake and the risk of CHD was based upon the results of 14 studies ( 2 on secondary prevention). The results of this work showed a reduced risk of $\mathrm{CHD}$ with increasing dietary fibre intake, too. The relative risk of CHD for persons in the highest quantile of dietary fibre intake was $0.78(95 \%$ CI $0.72 ; 0.84)$ compared with the lowest quantile of intake.

A recent analysis of the Zutphen Study that has been running for 40 years (but which is not included in the meta-analyses) confirmed these results, but also showed that the effect of dietary fibre intake decreases with increasing age [Streppel et al., 2008] (LOE IIb).

In spite of consistent evidence from several meta-analyses of observational studies, the proof from intervention studies is missing so far. This results in the following judgement:

On the basis of the available literature, the evidence regarding the primary prevention of $C H D$ by an increased dietary fibre intake is judged as probable.

Dietary Fibre from Cereal Products, Fruit and

Vegetables

In the combined analysis of data from 10 cohort studies [Pereira et al., 2004] (LOE IIa), differences in the effects of dietary fibre from cereal products, fruit or vegetables were shown. The intake of dietary fibre from cereal products was associated with a risk of $\mathrm{CHD}$ and fatal CHD that was lowered non-significantly by $10 \%$ (RR = $0.90 ; 95 \%$ CI $0.77 ; 1.07)$ and significantly by $25 \%$ (RR = $0.75 ; 95 \%$ CI $0.63 ; 0.91)$, respectively. For dietary fibre intake from fruit, the RR of CHD and fatal CHD was 0.84 (95\% CI 0.70; 0.99) and 0.70 (95\% CI 0.55; 0.89), respectively. Regarding dietary fibre intake from vegetables, no significant association was shown [Pereira et al., 2004] (LOE IIa).

According to the results of another meta-analysis, the consumption of dietary fibre from cereal products (4 individual studies) reduced the risk of CHD in the highest intake quantile by $16 \%(\mathrm{RR}=0.84,95 \%$ CI $0.62 ; 1.05)$, but this effect was not statistically significant [Anderson, 2005] (LOE IIa).

Another cohort study, which was not included in the 2 meta-analyses, showed a significant inverse association between dietary fibre intake from cereal products and the incidence of CHD, but not between dietary fibre intake from fruit or vegetables and the incidence of $\mathrm{CHD}$ [Mozaffarian et al., 2003] (LOE IIb).

As the risk reduction was not significant in some of the available meta-analyses, the evidence is judged as follows:

The results regarding the intake of dietary fibre from cereal products and fruit show with possible evidence that there is an inverse association with the risk of CHD; whereas regarding the intake of dietary fibre intake from vegetables, there is possible evidence that there is no association with the risk of CHD.

Soluble and Insoluble Dietary Fibre

In the combined analysis of data from 10 cohort studies [Pereira et al., 2004] (LOE IIa), there was a significant inverse association between the intake of soluble and insoluble dietary fibre and the risk of CHD. However, the risk-reducing effect regarding incident $\mathrm{CHD}$ and fatal $\mathrm{CHD}$ of increased intake of soluble dietary fibre $(\mathrm{RR}=$ 0.72 ; $95 \%$ CI $0.55 ; 0.93$ and $\mathrm{RR}=0.46$; 95\% CI 0.28; 0.74 , respectively) seems to be greater than the effect with insoluble dietary fibre $(\mathrm{RR}=0.90 ; 95 \% \mathrm{CI} 0.83 ; 0.97$ and $\mathrm{RR}=0.80 ; 95 \%$ CI 0.69; 0.92, respectively) [Pereira et al., 2004] (LOE IIa). This is also indicated by the findings of other cohort studies [Bazzano et al., 2003; Wu et al., 2003] (LOE IIb each).

There is possible evidence that an increased intake of both soluble and insoluble dietary fibre is associated with a reduced CHD risk, although soluble dietary fibre seems to have a greater effect than insoluble dietary fibre.

\section{Whole-Grain Products}

In a meta-analysis from 2005 , the risk of CHD was significantly reduced by $28 \%(\mathrm{RR}=0.72 ; 95 \%$ CI $0.49 ; 0.94)$ in persons with a high consumption of whole-grain products (highest quantile vs. lowest quantile) [Anderson, 2005] (LOE IIa). In another meta-analysis [Mellen et al., 2008] (LOE IIa) on the association between whole-grain products in the diet and the risk of cardiovascular diseases, 7 prospective cohort studies were included. The RR of CHD was $0.76(95 \%$ CI $0.69 ; 0.83)$ in the group with highest whole-grain product intake (2.5 portions per day) compared with the group with lowest intake ( 0.2 portions per day). Finally, also the most recent of the 3 meta-analyses including 12 cohort studies showed that persons with the highest compared to those with the lowest con- 
sumption of whole-grain products have a significantly lowered risk of $\mathrm{CHD}(\mathrm{RR}=0.81$; 95\% CI $0.75 ; 0.86)$ [Mente et al., 2009] (LOE IIa).

So far, the consistent significant results of the 3 metaanalyses of cohort studies have not been confirmed in intervention studies. Therefore, the evidence is judged as follows:

According to the results of the available studies, there is probable evidence regarding primary prevention of CHD by increasing the consumption of whole-grain products.

\subsection{Glycaemic Index and Glycaemic Load}

There are 2 meta-analyses on the association between GI or GL of the diet and the risk of CHD. In the most recent analysis [Mente et al., 2009] (LOE IIa), 5 prospective cohort studies were included; 2 of them were already the basis of the meta-analysis by Barclay et al. [2008] (LOE IIa). While Barclay et al. analysed the effects of GI and GL separately, Mente et al. determined only a common estimator. With regard to the different definitions of GI and GL, it appears to make sense to investigate both variables separately. Therefore, in the following the results are described separately for GI and GL.

\section{Glycaemic Index}

In the study by van Dam et al. [2000] (LOE IIb), no association could be detected between GI of a diet and the risk of CHD in men of the Zutphen Elderly Study. In contrast, Liu et al. [2000b] (LOE IIb) found a significantly increased risk of CHD in women of the Nurses' Health Study in the highest quintile of GI. The meta-analysis by Barclay et al. [2008] (LOE IIa) on the basis of these 2 studies showed a marginally significant positive association between GI and CHD (RR = 1.25; 95\% CI 1.00; 1.56; highest vs. lowest quantile). In 2007, 2 additional studies on this subject were published. While there was no association between GI and the risk of CHD in the study by Levitan et al. [2007] (LOE IIb) in a cohort of Swedish men, Beulens et al. [2007] (LOE IIb) showed a significantly increased risk of CHD with increasing GI in Dutch women.

\section{Glycaemic Load}

The first publication on the association between GL of a diet and the risk of CHD was the one by Liu et al. [2000b] (LOE IIb), which showed a significantly increased risk of $\mathrm{CHD}$ in women in the highest quintile of GL compared with those in the lowest quintile. In the study by van Dam [2000] (LOE IIb), presented in Barclay et al. [2008], the association between GL and the risk of CHD was not statistically significant. In the meta-analysis of the 2 cohort studies, a non-significant association between GL and the incidence of $\mathrm{CHD}(\mathrm{RR}=1.57 ; 95 \% \mathrm{CI} 0.87 ; 2.84$; highest vs. lowest quantile) [Barclay et al., 2008] (LOE IIa) was shown as well. No significant association between dietary GL and the risk of CHD could be demonstrated in either the study by Levitan et al. [2007] (LOE IIb) or the study by Beulens et al. [2007] (LOE IIb). In contrast, an analysis of the Nurses' Health Study [Halton et al., 2006] (LOE IIb) showed a significantly increased risk of CHD in women with the highest GL in their diet.

When considering all the studies, it is noticeable that significant associations were only observed in women. In the meta-analysis by Mente et al. [2009] (LOE IIa) in which GI and GL were analysed together, there were significant gender differences; GI and GL did not show a significant association in men $(\mathrm{RR}=1.06$; $95 \%$ CI 0.91 ; 1.20); but in women a high GI and a high GL were associated with an increased risk of CHD (RR $=1.50$; 95\% CI $1.29 ; 1.71)$.

Due to the inconsistency of the results of the individual studies, the evidence is judged as follows:

There is possible evidence that a high GI or a high GL in a diet increase the risk of CHD in women and possible evidence that there is no association in men.

\subsection{Need for Research regarding $C H D$}

Regarding the association between the intake of carbohydrates (total carbohydrates and polysaccharides) and the risk of CHD, there is no final conclusion whether the associations that partially have been observed can rather be explained by the lower consumption of fat or specific fatty acids at an increased carbohydrate intake. This could also be the cause of the contradictory results of the published meta-analysis [Jakobsen et al., 2009]. Due to the methodological problems, cohort studies cannot provide a definitive conclusion regarding cause-effect relations, so that additional intervention studies would be useful. However, in this case, too, an increased carbohydrate intake is accompanied by a lower intake of other energy sources, if constant body weight is required. The only intervention study that is available at present could not show a significant association between increased carbohydrate intake and the risk of CHD. 
The available studies on the influence of the consumption of mono- and disaccharides on the risk of CHD are far from being sufficient to allow a judgement of the association; there is an urgent need for additional research. Only 1 available study investigated the influence of sugarsweetened beverages.

Regarding the association between the consumption of dietary fibre and the risk of CHD, a great number of studies with good and relatively consistent evidence is available. However, it would be useful to examine the effect and its underlying mechanisms of action in intervention studies in more detail. Regarding the clarification of possibly different effects of soluble and insoluble dietary fibre and of dietary fibre from fruit and vegetables on the risk of $\mathrm{CHD}$, intervention studies would be useful as well. Likewise, confirming the observed inverse associations between whole-grain products and the risk of CHD in intervention studies is considered appropriate.

The results of the 5 available individual studies on the risk association between GI and GL of the diet and the occurrence of CHD are inconsistent. Therefore, it would be necessary to conduct further studies (cohort studies). So far, the significant risk-increasing associations in women are particularly based upon data from the Nurses Health Study. A meta-analysis should be performed separately on GI and GL in which the gender difference could be analysed separately for GI and GL as well.

\section{Quantity and Quality of Carbohydrate Intake and Primary Prevention of Cancer}

For evidence judgement regarding cancer an existing systematic collection of literature of the World Cancer Research Fund (WCRF) was used, supplemented by new studies appearing after the release of the collection. This systematic collection of literature (Systematic Literature Reviews [SLRs]) was performed on behalf of the WCRF by selected working groups and is attached as computer disk to the report of the WCRF [WCRF/AICR, 2007].

\subsection{Proportion of Carbohydrates}

So far only a few studies have investigated how carbohydrate intake influences the overall risk of cancer. In the Swedish Women's Lifestyle and Health Cohort Study such an analysis was performed. No association was found between the intake of energy-yielding nutrients including carbohydrates and the risk of cancer [Lagiou et al., 2007] (LOE IIb).

\section{Cancer of the Oesophagus}

Initially, the oesophagus is lined with squamous epithelium. This original epithelial tissue can change with age and transform into intestinal tissue structures. This process is limited to the lower part of the oesophagus. An important risk factor is abdominal obesity, which promotes reflux of chyme from the stomach into the oesophagus, which in turn is considered to be one of the causes of the tissue remodelling. Malignant tumours that develop from these new tissue structures are histologically adenocarcinomas. The risk factors for these adenocarcinomas are often investigated together with adenocarcinomas of the stomach. Though the incidence of oesophageal adenocarcinoma has been rising strongly in recent decades, squamous cell carcinoma still accounts for the majority of oesophageal carcinomas. The most important risk factors for squamous cell carcinoma are smoking tobacco and the consumption of alcohol [Siewert and Ott, 2007].

The SLR on oesophageal cancer [WCRF/AICR, 2007] refers to a cohort study which could not observe a significant association between carbohydrate intake and oesophageal carcinoma in a Japanese-American study population. In further literature research, no studies could be identified that have investigated carbohydrate intake prospectively regarding malignant tumours of the oesophagus.

\section{Cancer of the Stomach}

In the SLR on stomach cancer [WCRF/AICR, 2007], a total of 3 cohort studies on carbohydrate intake could be identified, the smallest of which had been conducted in South Korea and, with 44 new cases, detected an inverse association regarding carbohydrate intake. The 2 other studies did not show a risk relation. In a more recent Swedish cohort study in women, no risk relation between carbohydrate intake and stomach cancer could be observed [Larsson et al., 2006a] (LOE IIb).

\section{Cancer of the Colon or Colorectum ${ }^{2}$}

In the SLR on cancer of the colon or colorectum [WCRF/AICR, 2007], 5 cohort studies were presented that investigated the total intake of carbohydrates. These 5 studies did not show a risk relation. Several new study results were published after the SLR. While the Women's Health Initiative [Kabat et al., 2008] (LOE IIb) and a

\footnotetext{
2 The cancer site colorectum comprises colon and rectum.
} 
Swedish cohort study in women [Larsson et al., 2007] (LOE IIb) do not report a risk relation between carbohydrate intake and the risk of colorectal cancer, the Breast Cancer Detection Demonstration Project [Strayer et al., 2007] (LOE IIb) and the Multiethnic Cohort Study [Howarth et al., 2008] (LOE IIb) have observed an inverse association.

\section{Cancer of the Breast}

In the SLR on breast cancer [WCRF/AICR, 2007], the results of 8 cohort studies were described. Most of these studies did not show associations with carbohydrate intake, but there were also both positive and inverse risk relations. A meta-analysis by one of the SLR working groups, including 3 of these studies that showed a positive risk relation between cases of postmenopausal breast cancer and carbohydrate intake, resulted in a marginally significant association $(\mathrm{RR}=1.09$; $95 \% \mathrm{CI} 1.00 ; 1.18$ per $50 \mathrm{~g}$ carbohydrates/day). The analyses of cohort studies published after the SLR gave similar results. Apart from studies in which no risk relation was observed [Giles et al., 2006; Lajous et al., 2008; Larsson et al., 2009] (all LOE IIb), there was also 1 study from China with an increased risk with higher carbohydrate intake [Wen et al., 2009] (LOE IIb).

\section{Cancer of the Endometrium}

From the SLR on endometrial cancer [WCRF/AICR, 2007], 3 studies were identified. In these studies, no significant risk relations were found. The 2 papers that were published after the SLR did not find a risk relation either [Larsson et al., 2007; Cust et al., 2007] (both LOE IIb).

\section{Cancer of the Pancreas}

Results of 5 cohort studies were available from the SLR [WCRF/AICR, 2007]. Four of these studies could be included into a quantitative meta-analysis. The analysis did not reveal a significant risk relation between carbohydrate intake and pancreatic cancer $(\mathrm{RR}=0.95 ; 95 \%$ CI $0.89 ; 1.21$ per $50 \mathrm{~g}$ carbohydrates/day). The 5th cohort study and the cohort studies published after the SLR did not find a risk relation between carbohydrate intake and pancreatic cancer either [Nöthlings et al., 2007; Patel et al., 2007; Heinen et al., 2008; Jiao et al., 2009] (all LOE IIb).

The evidence regarding the influence of total carbohydrate intake on the occurrence of oesophageal cancer is judged as insufficient due to the low number of studies. There is possible evidence that there is no association between total carbohydrate intake and the risk of cancer of the stomach, breast, endometrium and pancreas. Due to the number of studies available, this also applies to cancer of the colorectum, even though 2 recent cohort studies indicate an inverse risk relation.

\subsection{Mono- and Disaccharides}

The SLR results [WCRF/AICR, 2007] regarding the intake of mono- and disaccharides ${ }^{3}$, sugar ${ }^{4}$ and sugarsweetened beverages were utilized for this evaluation.

\section{Cancer of the Oesophagus}

In the SLR [WCRF/AICR, 2007], no cohort study was mentioned that had exclusively investigated the association between oesophageal cancer and mono- and disaccharides, sugar as food as well as sugar-sweetened beverages. In the SLR, a cohort study was described that observed a non-significant reduction in the risk of squamous cell carcinoma in the upper digestive tract (mouth/ pharynx, larynx, oesophagus) in association with high sugar consumption. In the literature published after the SLR, no studies could be identified that exclusively investigated oesophageal cancer.

\section{Cancer of the Stomach}

In the SLR [WCRF/AICR, 2007], no prospective studies on the association between mono- and disaccharides or sugar-sweetened beverages and the development of stomach cancer were mentioned. More recent studies on the association between malignant tumours in the stomach and mono- and disaccharides or sugar-sweetened beverages could not be identified.

\section{Cancer of the Colon or Colorectum}

The association between the intake of sugar and the risk of malignant colorectal tumours was described in the main report of the WCRF [WCRF/AICR, 2007]. According to the WCRF, there is a possible positive risk relation. In the SLR [WCRF/AICR, 2007], 4 cohort studies on fructose were reported, 1 of which showed a significant positive association, and 2 studies on lactose which showed no risk relation. None of the 3 cohort studies in the SLR that investigated the risk relation between disaccharides and colorectal carcinoma showed a significant positive risk relation. Regarding sugar-sweetened beverages, only 1 cohort study from 1985 was mentioned, which did not

\footnotetext{
Intake determined from nutrition value tables for foods Sugar (sucrose) added to foods before consumption.
} 
show a risk relation [WCRF/AICR, 2007]. In a new study on this subject from 2008, sugar intake could not be associated with malignant tumours of the colorectum [Kabat et al., 2008] (LOE IIb). In a recently performed analysis of 13 cohort studies from the Pooling Project, the intake of sugar-sweetened beverages was not associated with the risk of colorectal cancer [Zhang et al., 2010] (LOE IIa).

\section{Cancer of the Breast}

In the SLR [WCRF/AICR, 2007], 3 cohort studies on the intake of lactose or sucrose or fructose were described. Neither the intake of lactose nor sucrose nor fructose was associated with the risk of breast cancer. The intake of fructose, glucose, and lactose was investigated in the Danish part of the EPIC Study regarding the risk of breast cancer. Here as well, no risk relation was observed [Nielsen et al., 2005] (LOE IIb). No cohort studies on the association with sugar-sweetened beverage consumption had been identified.

\section{Cancer of the Endometrium}

In the SLR [WCRF/AICR, 2007], 1 cohort study was described having investigated the association between sugar intake and malignant tumours of the endometrium. The study did not report a risk relation. After the publication of the SLR, 1 cohort study was identified that showed no risk relation [Cust et al., 2007] (LOE IIb). Neither cohort studies on the consumption of monosaccharides nor on the consumption of sugar-sweetened beverages had been identified.

\section{Cancer of the Pancreas}

In the SLR [WCRF/AICR, 2007], 1 cohort study was reported that investigated fructose intake with regard to malignant tumours of the pancreas. In this study there was no significantly increased risk with regard to high fructose intake. Two recent large U.S. studies (the Multiethnic Cohort Study and the NIH-AARP Study) investigated fructose intake; both found an increased risk of pancreatic cancer with high fructose intake [Nöthlings et al., 2007; Jiao et al., 2009] (both LOE IIb). Likewise, in the NIH-AARP Study, an increased risk with high glucose intake was observed [Jiao et al., 2009] (LOE IIb). In contrast, in the Netherlands Diet and Health Study, the intake of mono- and disaccharides was not associated with the risk of pancreatic cancer [Heinen et al., 2008] (LOE IIb). There were 3 cohort studies on sucrose intake in the SLR that did not report a risk relation [WCRF/ AICR, 2007].
In the SLR [WCRF/AICR, 2007], no cohort study reporting on the risk relation between malignant tumours of the pancreas and intake of sugar was identified. In studies that were published after the SLR, the quantity of sugar intake did not prove to be a risk factor for pancreatic cancer [Nöthlings et al., 2007; Jiao et al., 2009] (both LOE IIb). Likewise, the consumption of sweets or jam was no risk factors for malignant tumours of the pancreas [Larsson et al., 2006b] (LOE IIb).

The association between the consumption of sugarsweetened beverages and pancreatic cancer was investigated in a meta-analysis [Gallus et al., 2011] (LOE IIa). The analysis of 6 cohort studies did not show a change in the risk of pancreatic cancer in participants who consumed sugar-sweetened beverages compared to participants who did not $(\mathrm{RR}=1.05 ; 95 \%$ CI $0.94 ; 1.17)$. When several categories of sugar-sweetened beverage consumptions were analysed, a study of the Karolinska Institute [Larsson et al., 2006b] (LOE IIb) and a Chinese study including 60,000 men and women [Mueller et al., 2010] (LOE IIb) resulted in a positive risk relation between the intake of sugar-sweetened beverages and cancer of the pancreas.

The evidence regarding an association between the intake of monosaccharides and the risk of cancer of the oesophagus, colorectum and breast is judged as insufficient. The evidence regarding an association between the intake of disaccharides and the risk of cancer of the oesophagus and endometrium is judged as insufficient as well. There is possible evidence of a lack of a risk relation between the intake of disaccharides and the development of malignant tumours in the colorectum, breast and pancreas. There is also possible evidence regarding a lack of an association between the intake of sugar-sweetened beverages and cancer of the colorectum and pancreas. There is possible evidence of a positive association between the intake of monosaccharides and malignant tumours of the pancreas.

No cohort or intervention studies were identified on the association between the intake of monosaccharides and the risk of malignant tumours in the stomach and endometrium as well as between the intake of disaccharides and the risk of stomach cancer and between the intake of sugar-sweetened beverages and the risk of malignant tumours of the oesophagus, stomach, breast and endometrium. 


\subsection{Polysaccharides}

Starch is the most important polysaccharide in food. In the SLRs [WCRF/AICR, 2007], this type of carbohydrate was evaluated separately from the study results regarding cereal products in general and foods like bread, rice and pasta. The study results of the SLRs regarding refined grain products are described in this chapter as well.

\section{Cancer of the Oesophagus}

In the SLR [WCRF/AICR, 2007], no cohort study on the risk relation between starch intake and oesophageal cancer was included. In 3 cohort studies, the association between the consumption of refined grain products and the risk of carcinomas in the upper digestive tract was investigated and no risk relation was found. After the SLR publication, no additional studies on this subject were identified.

\section{Cancer of the Stomach}

In the SLR on stomach cancer [WCRF/AICR, 2007], a Dutch study was mentioned in which no association between starch intake and the risk of stomach cancer was observed. After the SLR publication, no additional studies on this subject were identified. Additionally, the SLR reported on 6 cohort studies that investigated the association between the intake of refined grain products and the risk of stomach cancer. In none of these studies was a significant risk relation detected.

\section{Cancer of the Colon or Colorectum}

The SLR [WCRF/AICR, 2007] found 3 cohort studies regarding starch intake; 2 of the studies did not reveal a risk relation, and 1 study showed higher starch consumption in participants with colorectal cancer. The consumption of rice, pasta and refined grain products was investigated in a total of 5 cohort studies. No risk relation could be observed. The 2 studies that investigated the intake of breakfast cereals did not find a risk relation. A study in the Multiethnic Cohort Study, published after the SLR on the intake of cereal products, found no risk relation either [Nomura et al., 2008] (LOE IIb).

\section{Cancer of the Breast}

The only cohort study that was mentioned in the SLR [WCRF/AICR, 2007] did not show a risk relation between the intake of starch and breast cancer. A more recent study did not show a risk relation with respect to the intake of polysaccharides either [Nielsen et al., 2005] (LOE IIb).
Cancer of the Endometrium

There were no intervention or cohort studies on the intake of cereal products presented in the SLR [WCRF/ AICR, 2007]. There was 1 cohort study on the consumption of refined grain products. The study did not show a risk relation. A more recent cohort study on the risk relation between cancer of the endometrium and starch intake did not show a risk relation [Cust et al., 2007] (LOE IIb).

\section{Cancer of the Pancreas}

One cohort study on the association between the intake of starch and the occurrence of pancreatic cancer was included in the SLR [WCRF/AICR, 2007]. This cohort study did not show a risk relation. Additionally, 3 cohort studies on the intake of cereal products in general were described in the SLR, all of which did not report a significant result. A more recent cohort study on pancreatic cancer [Jiao et al., 2009] (LOE IIb) did not show an association either.

There is insufficient evidence regarding the association between the intake of polysaccharides (starch) and the risk of cancer of the oesophagus, breast and endometrium. The evidence regarding a lack of a risk relation between the intake of polysaccharides and cancer of the stomach, colorectum and pancreas is judged as possible.

\subsubsection{Dietary Fibre}

At first, dietary fibre exert their effects locally in the stomach, colon and rectum. Potential indirect effects on other organs are mainly due to the metabolic effects of dietary fibre, especially due to their influence on glucose metabolism.

\section{Cancer of the Oesophagus}

Only 1 cohort study was described in the respective SLR [WCRF/AICR, 2007]. Without additional statistical information, the study reported a reduced risk of oesophageal cancer in association with higher intake of dietary fibre and cereal products. Because of this study, it was pointed out in the main report of the WCRF that high dietary fibre intake may lower the risk of oesophageal cancer. However, the WCRF experts based their association on further evidence coming from case-control studies, which are not considered in this guideline and thus are not taken into consideration. According to the WCRF, in particular the risk of oesophageal adenocarcinoma, which has been described above, might be reduced with increasing dietary fibre intake. Five out of 6 case-control 
studies indicated such a risk relation. No studies on this subject were identified after the SLR.

\section{Cancer of the Stomach}

Three cohort studies could be identified in the SLR [WCRF/AICR, 2007] that showed no association between dietary fibre intake and the risk of stomach cancer in either a meta-analysis or individually. One of the studies in particular also investigated the intake of dietary fibre from cereals, which showed an inverse but non-significant association with the risk of stomach cancer. In the EPIC $S t u d y$, higher dietary fibre intake from cereals, but not total dietary fibre intake or the intake of dietary fibre from other foods, was associated with a significantly reduced risk of stomach cancer [Mendez et al., 2007] (LOE IIb).

\section{Cancer of the Colon or Colorectum}

WCRF [2007] and DGE [2008] judged the evidence regarding an inverse association between colon cancer and dietary fibre intake as probable.

In the SLR [WCRF/AICR, 2007], different meta-analyses on colorectal cancer as well as colon cancer alone are quoted. Based on 10 available cohort studies, a reduced risk of colorectal cancer was calculated in the quantitative meta-analysis (RR per $10 \mathrm{~g}$ dietary fibre/day $=0.90 ; 95 \%$ CI $0.84 ; 0.97)$. In the 5 cohort studies on colon cancer, the risk assessment from the quantitative meta-analysis gave a similar result. The results of the cohort studies published from 2005 to 2008 were reported in the Nutrition Report 2008 of the DGE [DGE, 2008]. Three Asian cohort studies were cited. In the Chinese study, no association between the intake of dietary fibre and colorectal cancer was observed [Shin et al., 2006] (LOE IIb). The 2 Japanese studies showed an inverse relation [Otani et al., 2006; Wakai et al., 2007] (both LOE IIb). Further, the analysis of data from the Multiethnic Cohort Study resulted in a significant inverse association between the intake of dietary fibre and the risk of colorectal cancer only in men; in women the observed risk reduction was not significant [Nomura et al., 2007] (LOE IIb). A follow-up analysis of the EPIC Study confirmed the results of an earlier analysis, in which a significantly reduced risk had been observed with high dietary fibre intake [Bingham et al., 2005] (LOE IIb). The largest risk reduction regarding colorectal cancer in the EPIC Study was observed in association with the intake of fruit and cereal dietary fibre. The Pooling Project including 13 cohort studies, which have partially been conducted in Europe, with over 8,000 incident cases of colorectal carcinoma and over 700,000 study participants, resulted in a different estimate of the associations regard- ing the intake of dietary fibre [Park et al., 2005] (LOE Ib). In this study, only a non-significantly lowered risk of colorectal carcinoma with dietary fibre intake could be observed. In a further analysis regarding non-linear associations, an intake of less than $10 \mathrm{~g} /$ day of dietary fibre was associated with a higher risk. In the NIH-AARP Study, which is numerically comparable with the EPIC Study, no significant inverse association between total intake of dietary fibre and the risk of colorectal carcinoma was observed; however, the intake of dietary fibre from cereals was associated with a reduced risk of disease [Schatzkin et al., 2007] (LOE IIb). An analysis of the observational arm of the Women's Health Initiative including 158,200 women, which was published after the Nutrition Report 2008 [DGE, 2008], did not confirm an inverse risk relation between dietary fibre intake and colorectal cancer: between an intake of about $10 \mathrm{~g} /$ day and an intake of about $21 \mathrm{~g} /$ day, no difference in risk was observed [Kabat et al., 2008] (LOE IIb). The particular role of cereals as a source of dietary fibre for the development of these malignant tumours, which had already been discussed in the Nutrition Report 2008 [DGE, 2008], could not be confirmed in this study. The 3 cohort studies in the SLR [WCRF/AICR, 2007] on the intake of refined grain products did not show a risk relation.

\section{Cancer of the Breast}

In the SLR [WCRF/AICR, 2007], 4 studies on dietary fibre intake were analysed in the meta-analysis, 1 of which showed a risk reduction with increased dietary fibre intake. However, the overall estimate did not indicate a risk relation. The 3 cohort studies on the intake of cereal products high in dietary fibre did not show a risk association either [WCRF/AICR, 2007]. The studies performed after the publication of the SLR only partially supported the previous assessment that dietary fibre does not influence the risk of breast cancer. While the intake of dietary fibre did not have an effect both in a Swedish and in a British study [Suzuki et al., 2008; Cade et al., 2007] (both LOE IIb), the NIH-AARP Study showed an inverse relation [Park et al., 2009] (LOE IIb). This inverse risk relation regarding the intake of watersoluble dietary fibre could specifically be observed in oestrogen/progesterone-negative tumours. In general, the source of dietary fibre did not influence the observed reduced relative risk.

Cancer of the Endometrium

There is only 1 cohort study mentioned in the SLR [WCRF/AICR, 2007] on the relation between dietary fi- 
bre intake and endometrial cancer. This study did not show a risk relation. The analysis of the EPIC Study that was conducted after the publication of the SLR did not result in a respective risk relation either [Cust et al., 2007] (LOE IIb). Cereal products rich in dietary fibre were not associated with the risk of endometrial cancer in the cohort study already mentioned in the SLR [WCRF/AICR, 2007].

\section{Cancer of the Pancreas}

In the SLR [WCRF/AICR, 2007], 1 cohort study was reported that did not show an association between the intake of dietary fibre and the risk of pancreatic cancer. An investigation on the intake of cereal products high in dietary fibre did not show a risk association either [WCRF/AICR, 2007]. More recent prospective studies on the risk relation between malignant tumours of this organ and dietary fibre intake could not be identified.

The evidence regarding the association between the intake of dietary fibre and the development of cancer of the oesophagus, endometrium, stomach and pancreas is insufficient. There is possible evidence that there is no association between the intake of dietary fibre and the risk of cancer of the breast. There is probable evidence that there is a risk-reducing association between the intake of dietary fibre from cereal products and the risk of colorectal cancer, and there is possible evidence regarding total intake of dietary fibre. High intake of dietary fibre from cereal products lowers the risk of stomach cancer with possible evidence.

\subsection{Glycaemic Index and Glycaemic Load}

GL is highly correlated with carbohydrate intake in total [van Bakel et al., 2009]. Because of this, there should be no major differences between study results that either assess carbohydrate intake or GL. The evidence regarding the association between GI and GL and the risk of cancer was judged in the Nutrition Report 2008 [DGE, 2008]. In this report, insufficient evidence was found regarding almost all malignant tumours due to inconsistent or a lack of study results. Based on several study results that showed no risk association, the strength of the evidence was classified as 'possible' for a lack of risk relations regarding cancer of the stomach and colorectum. In the SLRs [WCRF/AICR, 2007], the risk relation with respect to GI and GL was not presented individually for each cancer site. The association between GI and GL, respectively, and the risk of malignant tumours was examined more detailed in 3 meta-analyses [Barclay et al., 2008] (LOE IIa), [Gnagnarella et al., 2008] (LOE IIa/IIIa), [Mulholland et al., 2008a, b] (both LOE IIa/IIIa). Although all of these meta-analyses were published in the same year, different studies were included in each of them. A difficulty regarding the evaluation of these metaanalyses is that, in part, the summarised risk assessments are not numerically specified, but only presented graphically.

In addition to the investigation of many individual cancer sites, cancer in general was also investigated in the $N I H$-AARP Study. In men a moderate but significant risk increase with increasing GI and a risk reduction with increasing GL was observed. In women there was no risk relation regarding GI, but a risk reduction regarding $\mathrm{GL}$ [George et al., 2009] (LOE IIb).

\section{Cancer of the Oesophagus}

In the SLR [WCRF/AICR, 2007], the risk relation between GI or GL and the risk of oesophageal cancer was not presented. Also, the 3 meta-analyses mentioned above did not indicate any study that investigated the risk relation between GI or GL and oesophageal cancer. More recently, in women, the risk of cancer of the oesophagus was neither associated with GI nor with GL in the $\mathrm{NIH}$ AARP Study. However, in men an increase in risk with increasing GI was observed [George et al., 2009] (LOE IIb).

\section{Cancer of the Stomach}

In the SLR [WCRF/AICR, 2007], the risk relation between GI or GL and the risk of stomach cancer was not presented. A Swedish study on malignant tumours of the stomach did not reveal a risk relation with GI or GL [Larsson et al., 2006a] (LOE IIb). However, the NIH-AARP Study showed an increased risk for this cancer site in men regarding high GI, but not in women regarding GL.

\section{Cancer of the Colon or Colorectum}

Risk assessment in the meta-analyses on the association between GI and colorectal cancer resulted in a significant positive association $(\mathrm{OR}=1.10 ; 95 \% \mathrm{CI} 1.00 ; 1.12)$ in Barclay et al. [2008] (LOE IIa) (data of 5 cohort studies were included), but in Gnagnarella et al. [2008] (LOE IIa/ IIIa) and in Mulholland et al. [2009] (LOE IIa/IIIa) no significant risk association was observed. More recent studies gave inconsistent results. While the Netherlands Diet and Health Study [Weijenberg et al., 2008] (LOE IIb) 
and the observational study of the Women's Health Initiative [Kabat et al., 2008] (LOE IIb) did not report a risk relation between GI and cancer of the colorectum, higher GI was associated with a significant increase in risk in men and a trend towards an increased risk in women in the NIH-AARP Study [George et al., 2009] (LOE IIb). Regarding GL, the Multiethnic Cohort Study [Howarth et al., 2008] (LOE IIb) and the NIH-AARP Study (men only; [George et al., 2009]) (LOE IIb) showed a risk reduction with increasing GL. Both the Netherlands Diet and Health Study [Weijenberg et al., 2008] (LOE IIb) and the observational study of the Women's Health Initiative [Kabat et al., 2008] (LOE IIb) did not find a risk relation with GL. This also applied to women in the NIH-AARP Study [George et al., 2009] (LOE IIb).

\section{Cancer of the Breast}

In the meta-analysis of Barclay et al. [2008] (LOE IIa) including 8 cohort studies, no significant risk relation was found between GI and the risk of breast cancer $(\mathrm{RR}=1.06 ; 95 \% \mathrm{CI} 0.98 ; 1.15)$ or $\mathrm{GL}(\mathrm{RR}=0.99 ; 95 \% \mathrm{CI}$ 0.94; 1.06). Gnagnarella et al. [2008] (LOE IIa/IIIa) did not calculate a significant risk relation with GI or GL in their meta-analysis either including 11 study results from cohort studies. Mulholland et al. [2008a] (LOE IIa/IIIa) did not find a significant relative risk in their meta-analysis including 6 cohort studies each on the association between premenopausal and postmenopausal risk of breast cancer and GI, 10 studies on the association between premenopausal breast cancer and GL and 6 studies on the association between postmenopausal breast cancer and GL. The NIH-AARP Study did not show a risk relation between GI and GL and cancer of the breast [George et al., 2009] (LOE IIb). A Swedish study also reported a lack of risk relations [Larsson et al., 2009] (LOE IIb). In a study from Shanghai, no risk relation was found in the entire group, but for premenopausal women, an increased risk with increasing GL was found [Wen et al., 2009] (LOE IIb).

\section{Cancer of the Endometrium}

The meta-analyses including up to 5 cohort studies did not show a significant risk association between cancer of the endometrium and GI, but between cancer of the endometrium and GL [Barclay et al., 2008] (LOE IIa), [Gagnarella et al., 2008] (LOE IIa/IIIa), [Mulholland et al., 2008b] (LOE IIa/IIIa). These findings are in contrast with the results of the NIH-AARP Study, which did not show a risk relation regarding either GI or GL [George et al., 2009] (LOE IIb).

\section{Cancer of the Pancreas}

In the meta-analyses by Barclay et al. [2008] (LOE IIa), Gnaglarella et al. [2008] (LOE IIa/IIIa) and Mulholland et al. [2009] (LOE IIa/IIIa) including up to 6 cohort studies, the association between the risk of pancreatic cancer and GI or GL was investigated. There was no risk association regarding either GI or GL. The studies conducted afterwards, the Netherlands Diet and Health Study (only GL) and the NIH-AARP Study, did not observe a risk association regarding GI and GL [Heinen et al., 2008; Jiao et al., 2009] (both LOE IIb). However, in another analysis of the NIH-AARP Study, an inverse association was observed in women regarding GL [George et al., 2009] (LOE IIb).

The evidence regarding an association between GI and the risk of cancer of the oesophagus and the stomach is judged as insufficient. There is possible evidence that there is a positive risk association with GI regarding cancer of the colorectum. There is possible evidence that there is no relation between GI and the risk of cancer of the endometrium, breast and pancreas.

The evidence regarding an association between GL and the risk of cancer of the oesophagus and the stomach is judged as insufficient, too. Regarding the risk of cancer of the endometrium, there is possible evidence that there is a positive risk relation with GL. There is possible evidence that there is no relation between GL and the risk of cancer of the colorectum and pancreas. The evidence regarding a lack of a risk relation between $G L$ and breast cancer is judged as probable.

\subsection{Need for Research regarding Cancer}

Further research regarding cancer should basically comprise a systematic evaluation of the existing cohort studies. This applies to all aspects of carbohydrate intake that have been subject of this guideline. Particularly noteworthy is that there is a lack of prospective studies that have investigated the association between the intake of dietary fibre and pancreatic cancer. This lack of data is inexplicable because of the potential importance of dietary fibre intake for the prevention of cancer. Additionally, more data analyses should be performed with regard to the overall risk of cancer. These analyses are less interesting from an aetiological point of view; however, they describe the impact of changes in carbohydrate intake on the occurrence of cancer in general. To evaluate the effect of whole-grain cereal intake, it would be preferable to examine foods high in whole-grain regarding their meta- 
bolic effects in intervention studies in more detail. This might also allow conclusions on metabolic processes involved in cancer.

\section{Summary of the Results of the Guideline on Carbohydrate Intake}

\section{Total Carbohydrate Intake}

Regarding the relation between total carbohydrate intake and the risk of the investigated chronic diseases, with different strength of the evidence, there is either no association detectable, or the evidence is insufficient (table 3). Dyslipoproteinaemias are an exception thereof. There is convincing evidence that a high carbohydrate intake at the expense of total intake of fat and saturated fatty acids is associated with reduced concentrations of total and LDL cholesterol as well as HDL cholesterol. There is also convincing evidence that a high carbohydrate intake promotes an increase in the triglyceride concentration, independent of the quality of fatty acids in food. There is convincing evidence that a high carbohydrate intake at the expense of polyunsaturated fatty acids is associated with increased plasma concentrations of total and LDL cholesterol and reduced plasma concentration of HDL cholesterol.

\section{Intake of Mono- and Disaccharides and}

Sugar-Sweetened Beverages

Regarding the intake of mono- and disaccharides, the evidence for an association with the respective diseases is either insufficient, or there is evidence that there is no association. Possible evidence for a positive risk relation is only evident regarding the intake of monosaccharides and the risk of pancreatic cancer. High consumption of sugar-sweetened beverages increases the risk of obesity with probable evidence in adults and with possible evidence in children. The consumption of sugar-sweetened beverages also increases the risk of type 2 diabetes mellitus with probable evidence and increases the risk of metabolic syndrome with possible evidence.

\section{Polysaccharide Intake}

Regarding the intake of polysaccharides and starch, there is insufficient evidence for a risk relation with respect to almost all diseases.

\section{Dietary Fibre Intake}

With probable evidence, high total dietary fibre intake lowers the risk of obesity in adults, as well as of hypertension and CHD. There is possible evidence that a high total dietary fibre intake lowers the risk of dyslipoproteinaemia (by lowering total and LDL cholesterol concentrations) and of colorectal cancer. High intake of dietary fibre from cereal products lowers the risk of type 2 diabetes mellitus and of colorectal cancer with probable evidence; the risk of CHD and stomach cancer is lowered with possible evidence. If only whole-grain products are considered, there is probable evidence that a high intake reduces the risk of type 2 diabetes mellitus, hypertension and CHD. A high intake of whole-grain products lowers the concentrations of total and LDL cholesterol with convincing evidence.

\section{GI and GL}

The analysis showed that a high-GI diet increases the risk of obesity (in women), type 2 diabetes mellitus, CHD (in women) and colorectal cancer with possible evidence. There is probable evidence that a high-GI diet also increases the total cholesterol concentration. With respect to a positive association with GL, there is only possible evidence regarding the risk of cancer of the endometrium and the risk of CHD (women) and probable evidence regarding triglyceride concentrations.

\section{Need for Research}

Although the number of studies on the association between carbohydrate intake and the risk of diseases has substantially increased in recent years, and also their scope and quality have further improved, many questions cannot be answered conclusively. There are no randomised controlled intervention studies for many questions. However, more and more important information is provided by prospective cohort studies. For the individual components, there are often only a few or little reliable study results available regarding the association between the intake of carbohydrates and the prevention of nutrition-related diseases. Thus, there is a considerable need for research. Particularly serious is the lack of long-term intervention studies with defined final outcomes, which have the highest level of evidence. However, these studies are especially complex and difficult to perform, so that for some time it will be necessary to mainly use intervention studies with surrogate markers or intermediary markers and cohort studies.

When studying carbohydrate intake in association with the emergence of chronic diseases it must not be forgotten that the overall risk is determined only by the interaction of the various food components, and therefore, the importance of the individual components should not be overestimated. However, there is only a limited number of studies with sufficient investigation of food ex- 
Table 3. Summary of the evidence regarding the association between carbohydrate intake and primary prevention of certain nutrition-related diseases

\begin{tabular}{|c|c|c|c|c|c|c|c|c|}
\hline Increase in: & $\begin{array}{l}\text { Carbohydrate } \\
\text { proportion }\end{array}$ & $\begin{array}{l}\text { Monosac- } \\
\text { charides }\end{array}$ & $\begin{array}{l}\text { Disaccha- } \\
\text { rides }\end{array}$ & $\begin{array}{l}\text { Sugar- } \\
\text { sweetened } \\
\text { beverages }\end{array}$ & $\begin{array}{l}\text { Polysac- } \\
\text { charides }\end{array}$ & $\begin{array}{l}\mathrm{DF} / \\
\text { whole-grain products }\end{array}$ & GI & GL \\
\hline \multicolumn{9}{|l|}{ Obesity } \\
\hline Adults & ০০ & $\sim$ & $\sim$ & $\uparrow \uparrow$ & - & $\begin{array}{l}\text { total DF: } \downarrow \\
\text { whole-grain products: } \downarrow\end{array}$ & $\uparrow \mathrm{h} / \sim^{\mathrm{i}}$ & O \\
\hline Children & ○ & $\sim$ & $\sim$ & $\uparrow$ & - & $\begin{array}{l}\text { total DF: } \bigcirc \\
\text { whole-grain products: } \sim\end{array}$ & $\sim$ & $\sim$ \\
\hline Type 2 diabetes mellitus & ○૦ & $\sim$ & $\sim / 00^{\mathrm{e}} / \mathrm{O}^{\mathrm{f}}$ & $\uparrow \uparrow$ & $\sim$ & $\begin{array}{l}\text { total DF: } \bigcirc \\
\text { whole-grain products: } \downarrow \\
\text { DF from cereal products: } \downarrow\end{array}$ & $\uparrow$ & $\bigcirc$ \\
\hline \multicolumn{9}{|l|}{ Dyslipoproteinaemia } \\
\hline Total cholesterol concentration & $\downarrow \downarrow^{\mathrm{a}}, \uparrow \uparrow \uparrow^{\mathrm{b}}$ & $\sim$ & $\sim$ & $\sim$ & $\sim$ & $\begin{array}{l}\text { total DF: } \downarrow \\
\text { whole-grain products: } \downarrow \downarrow\end{array}$ & $\uparrow \uparrow$ & $\sim$ \\
\hline LDL cholesterol concentration & $\downarrow \downarrow^{\mathrm{a}}, \uparrow \uparrow \uparrow^{\mathrm{b}}$ & $\sim$ & $\sim$ & $\sim$ & $\sim$ & $\begin{array}{l}\text { total DF: } \downarrow \\
\text { whole-grain products: } \downarrow \downarrow\end{array}$ & $\sim$ & $\sim$ \\
\hline HDL cholesterol concentration & $\downarrow \downarrow^{\mathrm{a}}, \downarrow \downarrow^{\mathrm{b}}$ & $\sim$ & $\sim$ & $\sim$ & $\sim$ & $\begin{array}{l}\text { total DF: } \bigcirc \bigcirc \\
\text { whole-grain products: } \bigcirc \bigcirc \bigcirc\end{array}$ & 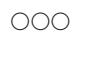 & $\sim$ \\
\hline Triglyceride concentration & $\uparrow \uparrow \uparrow$ & $\bigcirc^{c}$ & $\sim$ & $\sim$ & $\sim$ & $\begin{array}{l}\text { total DF: } \bigcirc \circ \bigcirc \\
\text { whole-grain products: } \bigcirc ০ \bigcirc\end{array}$ & ○ & $\uparrow \uparrow$ \\
\hline Hypertension & ○ & $\mathrm{O}^{\mathrm{d}}$ & $\mathrm{O}^{\mathrm{g}}$ & O & $\sim$ & $\begin{array}{l}\text { total DF: } \downarrow \\
\text { whole-grain products: } \downarrow\end{array}$ & $\sim$ & $\sim$ \\
\hline Metabolic syndrome & $\sim$ & - & - & $\uparrow$ & - & $\begin{array}{l}\text { total DF: } \bigcirc \\
\text { whole-grain products: } \sim\end{array}$ & - & - \\
\hline CHD & O & $\sim$ & $\sim$ & $\sim$ & $\sim$ & $\begin{array}{l}\text { total DF: } \downarrow \\
\text { whole-grain products: } \downarrow \\
\text { DF from cereal products: } \downarrow\end{array}$ & $\uparrow \mathrm{h} / \mathrm{O}^{\mathrm{i}}$ & $\uparrow \mathrm{h} / \mathrm{O}^{\mathrm{i}}$ \\
\hline \multicolumn{9}{|l|}{ Cancer } \\
\hline Oesophagus & $\sim$ & $\sim$ & $\sim$ & - & $\sim$ & $\sim$ & $\sim$ & $\sim$ \\
\hline Stomach & O & - & - & - & $\bigcirc$ & $\begin{array}{l}\text { total DF: } \sim \\
\text { DF from cereal products: } \downarrow\end{array}$ & $\sim$ & $\sim$ \\
\hline Colorectum & O & $\sim$ & O & O & $\bigcirc$ & $\begin{array}{l}\text { total DF: } \downarrow \\
\text { DF from cereal products: } \downarrow\end{array}$ & $\uparrow$ & $\bigcirc$ \\
\hline Breast & O & $\sim$ & O & - & $\sim$ & 0 & $\bigcirc$ & ০ \\
\hline Endometrium & O & - & $\sim$ & - & $\sim$ & $\sim$ & O & $\uparrow$ \\
\hline Pancreas & O & $\uparrow$ & 0 & O & ○ & $\sim$ & O & ○ \\
\hline
\end{tabular}

$\mathrm{DF}=$ Dietary fibre ${ }^{\mathrm{a}}$ At increased carbohydrate proportion at the expense of total fat or saturated fatty acids, respectively. ${ }^{\mathrm{b}}$ At increased carbohydrate proportion at the expense of polyunsaturated fatty acids. ${ }^{\mathrm{c}}$ At fructose up to $100 \mathrm{~g} / \mathrm{d}$, other monosaccharides $\sim$. ${ }^{\mathrm{d}}$ At long-term fructose intake. ${ }^{\mathrm{e}}$ Sucrose. ${ }^{\mathrm{f}}$ Lactose. ${ }^{\mathrm{g}}$ Long-term sucrose intake. ${ }^{\mathrm{h}}$ Women. ${ }^{\mathrm{i}}$ Men.

The number of arrows only indicates the level of evidence of the data and not the extent of the risk.

$\uparrow=$ Possible evidence, risk-enhancing; $\uparrow \uparrow=$ probable evidence, risk-enhancing; $\uparrow \uparrow \uparrow=$ convincing evidence, risk-enhancing.

$\downarrow=$ Possible evidence, risk-reducing; $\downarrow \downarrow=$ probable evidence, risk-reducing; $\downarrow \downarrow \downarrow$ = convincing evidence, risk-reducing.

$\mathrm{O}=$ Possible evidence, no association; $\bigcirc \bigcirc=$ probable evidence, no association; $\bigcirc \bigcirc \bigcirc=$ convincing evidence, no association .

$\sim=$ Insufficient evidence.

- = No study identified. 
change relations. Of special practical importance is the mutual exchange of carbohydrates and fat, which are the most important energy-yielding nutrients in quantitative terms. Especially in this respect, very different effects may occur depending on the ratio and type of carbohydrate and fat components in the diet [see also DGE, 2011b]. The exchange of carbohydrates for protein is potentially interesting as well and might modify the risk of nutrition-related diseases. However, there have only been a few studies in this area yet.

\section{Implementation of the Guideline on Carbohydrate Intake}

The implementation of the evidence-based guideline on carbohydrate intake in healthy individuals refers to those results for which the strength of the evidence regarding the association was judged as 'convincing' or 'probable'.

The present evidence-based guideline regarding carbohydrate intake and primary prevention of the diseases considered here has shown that the quality rather than the quantity of carbohydrate intake is important for the primary prevention of nutrition-related diseases. Based on the present results, it is possible to make recommendations that support the previous DGE recommendations on the choice of foods regarding the quality of carbohydrates [Stehle et al., 2005; DGE, 2010].

Due to the results of this guideline, great potential regarding the prevention of nutrition-related diseases is seen in a high intake of dietary fibre. The total intake of dietary fibre and especially the intake of whole-grain products, which are foods high in dietary fibre, should be increased, as this reduces the risk of various nutritionrelated diseases. On the other hand, the consumption of sugar-sweetened beverages should be limited, because they increase the risk of obesity and diabetes. These 2 main results should be emphasised in future nutrition counselling.

\section{Increase in Dietary Fibre Intake}

The preventive potential of dietary fibre should be exploited by increasing the consumption in the general public. According to the data of the National Nutrition Survey II [MRI, 2008], the intake of dietary fibre is below the guiding value of at least $30 \mathrm{~g} /$ day in $75 \%$ of all women and $68 \%$ of all men in Germany. In women, the median intake is $23 \mathrm{~g} / \mathrm{day}$, in men it is $25 \mathrm{~g} /$ day.

Dietary fibre intake may be increased by choosing the whole-grain variants of bread, pasta and other cereal products. When baking in private homes, dietary fibre intake can be increased by using cereal flour products with a high flour type number. Considering current habits and preferences in taste, moderate changes may be chosen: it is possible to increase dietary fibre intake considerably by exchanging half of the wheat flour type 405/550 for wheat whole-grain flour, by using wheat flour type 1050 or by partially using rye flour for savoury pastries.

The preferable regular consumption of foods high in dietary fibre such as muesli, legumes, whole-grain pasta in exchange for processed breakfast cereals and refined grain products, which is based on the present guideline and previous DGE recommendations on the choice of foods, usually is accompanied by a reduced GI of the diet. However, sometimes foods high in dietary fibre may also have a high GI, for example, whole-grain bread made from finely milled whole-grain flour in contrast to bread made from coarsely milled or whole grains. To date there is only possible evidence regarding a risk-increasing effect of high GI on some nutrition-related diseases. Therefore, no recommendations are made in that respect.

Dietary fibre intake can also be enhanced by an increased consumption of fruit and vegetables. Due to their high water content, fruit and vegetables belong to the foods with low energy density; at the same time, they are high in vitamins, minerals, dietary fibre and phytochemicals. Therefore, an increased consumption of fruit and vegetables is generally recommended because of their positive effects on human health [DGE, 2007].

\section{Reduced Consumption of Sugar-Sweetened Beverages}

In adolescents and young adults in Germany, the consumption of sugar-sweetened beverages and hence the intake of added sugar is particularly high [MRI, 2008]. Especially in these age groups, the risk of obesity could be lowered by a reduced consumption of sugar-sweetened beverages. Due to their content of mono- and disaccharides, sugar-sweetened beverages increase energy intake, unless there is a compensation through omitting other sugar-containing foods, which could explain the risk-increasing effect regarding obesity. This could also be the reason for the risk-increasing effect regarding diabetes. The consumption of sugar-sweetened beverages should be reduced by promoting the supply and the consumption of sugar-free, energy-free or low-energy alternatives. According to the DGE recommendations on the choice of foods, drinking water or mineral water and sugar-free herbal and fruit teas are the best alternatives [Stehle et al., 2005; DGE, 2010]. 


\section{Disclosure Statement}

At the time when the work for this publication was performed the author Anja Brönstrup was employed with the German Nutrition Society (DGE e.V.). Currently she is employed with the European Food Safety Authority (EFSA) in its Nutrition Unit that provides scientific and administrative support to the NDA Panel. The present article is published under the sole responsibil- ity of the author(s) and may not be considered as an EFSA scientific output. The positions and opinions presented in this article are those of the author(s) alone and are not intended to represent the views or scientific works of EFSA. To know about the views or scientific outputs of EFSA, please consult its website under http://www.efsa.europa.eu. The remaining authors have nothing to disclose.

\section{References}

Adams T, Rini A: Predicting 1-year change in body mass index among college students. J Am Coll Health 2007;55:361-365.

Albala C, Ebbeling CB, Cifuentes M, et al: Effects of replacing the habitual consumption of sugar-sweetened beverages with milk in Chilean children. Am J Clin Nutr 2008;88:605-611.

Alberti KG, Zimmet P, Shaw J, IDF Epidemiology Task Force Consensus Group: The metabolic syndrome - a new worldwide definition. Lancet 2005;366:1059-1062.

-Aller R, de Luis DA, Izaola O, et al: Effect of soluble fiber intake in lipid and glucose levels in healthy subjects: a randomized clinical trial. Diabetes Res Clin Pract 2004;65:7-11.

-Alonso A, Beunza JJ, Bes-Rastrollo M, et al: Vegetable protein and fiber from cereal are inversely associated with the risk of hypertension in a Spanish cohort. Arch Med Res 2006; 37:778-786

Anderson JW: Whole-grains intake and risk for coronary heart disease; in Marquart L, Slavin JL, Fulcher G (eds): Whole grain foods in health and disease. St Paul, Minn: American Association of Cereal Chemists, 2005, pp 187-200.

-Anderson JW, Garrity TF, Wood CL, et al: Prospective, randomized, controlled comparison of the effects of low-fat and low-fat plus high-fiber diets on serum lipid concentrations. Am J Clin Nutr 1992;56:887-894.

-Archer SL, Liu K, Dyer AR, et al: Relationship between changes in dietary sucrose and high density lipoprotein cholesterol: the CARDIA study. Coronary Artery Risk Development in Young Adults. Ann Epidemiol 1998;8: 433-438.

-Ascherio A, Hennekens C, Willett WC, et al: Prospective study of nutritional factors, blood pressure, and hypertension among US women. Hypertension 1996;27:1065-1072.

-Ascherio A, Rimm EB, Giovannucci EL, et al: A prospective study of nutritional factors and hypertension among US men. Circulation 1992;86:1475-1484.

Aston LM, Stokes CS, Jebb SA: No effect of a diet with a reduced glycaemic index on satiety, energy intake and body weight in overweight and obese women. Int J Obes (Lond) 2008;32: $160-165$.

Barclay AW, Petocz P, McMillan-Price J, et al: Glycemic index, glycemic load, and chronic disease risk - a meta-analysis of observational studies. Am J Clin Nutr 2008;87:627-637.

Barclay AW, Flood VM, Rochtchina E, et al: Glycemic index, dietary fiber, and risk of type 2 diabetes in a cohort of older Australians. Diabetes Care 2007;30:2811-2813.

-Bazzano LA, Song Y, Bubes V, et al: Dietary intake of whole and refined grain breakfast cereals and weight gain in men. Obes Res 2005; 13:1952-1960.

-Bazzano LA, He J, Ogden LG, et al: National Health and Nutrition Examination Survey Epidemiologic Follow-up Study. Dietary fiber intake and reduced risk of coronary heart disease in US men and women: the National Health and Nutrition Examination Survey I Epidemiologic Follow-up Study. Arch Intern Med 2003;163:1897-1904.

Berkey CS, Rockett HR, Field AE, et al: Sugaradded beverages and adolescent weight change. Obes Res 2004;12:778-788.

Berkey CS, Rockett HR, Field AE, et al: Activity, dietary intake, and weight changes in a longitudinal study of preadolescent and adolescent boys and girls. Pediatrics 2000;105:E56.

Bes-Rastrollo M, Sanchez-Villegas A, GomezGracia E, et al: Predictors of weight gain in a Mediterranean cohort: the Seguimiento Universidad de Navarra Study 1. Am J Clin Nutr 2006;83:362-370.

-Beulens JWJ, de Bruijne LM, Stolk RP, et al: High dietary glycemic load and glycemic index increase risk of cardiovascular disease among middle-aged women. A population-based follow-up study. J Am Coll Cardiol 2007;50 14-21.

Bingham SA, Norat T, Moskal A, et al: Is the association with fiber from foods in colorectal cancer confounded by folate intake? Cancer Epidemiol Biomarkers Prev 2005;14:15521556.

Blum JW, Jacobsen DJ, Donnelly JE: Beverage consumption patterns in elementary school aged children across a two-year period. J Am Coll Nutr 2005;24:93-98.

Bogaert N, Steinbeck KS, Baur LA, et al: Food, activity and family - environmental versus biochemical predictors of weight gain in children. Eur J Clin Nutr 2003;57:12421249.

Boreham C, Twisk J, van Mechelen W, et al: Relationships between the development of bio- logical risk factors for coronary heart disease and lifestyle parameters during adolescence: The Northern Ireland Young Hearts Project. Public Health 1999;113:7-12.

Bouché C, Rizkalla SW, Luo J, et al: Five-week, low-glycemic index diet decreases total fat mass and improves plasma lipid profile in moderately overweight nondiabetic men. Diabetes Care 2002;25:822-828.

Boyd NF, Cousins M, Beaton M, et al: Quantitative changes in dietary fat intake and serum cholesterol in women: results from a randomized, controlled trial. Am J Clin Nutr 1990;52:470-476

-Brown L, Rosner B, Willett WW, Sacks FM: Cholesterol-lowering effects of dietary fiber: a meta-analysis. Am J Clin Nutr 1999;69:3042.

Brown RC, Cox CM: Effects of high fat versus high carbohydrate diets on plasma lipids and lipoproteins in endurance athletes. Med Sci Sports Exerc 1998;30:1677-1683.

Buyken AE, Cheng G, Gunther AL, et al: Relation of dietary glycemic index, glycemic load, added sugar intake, or fiber intake to the development of body composition between ages 2 and 7 y. Am J Clin Nutr 2008; 88:755-762

Cade JE, Burley VJ, Greenwood DC; UK Women's Cohort Study Steering Group: Dietary fibre and risk of breast cancer in the UK Women's Cohort Study. Int J Epidemiol 2007;36:431-438.

Cao Y, Maugre DT, Pelkman CL, et al: Effects of moderate (MF) versus lower fat (LF) diets on lipids and lipoproteins: a meta-analysis of clinical trials in subjects with and without diabetes. J Lipidol 2009;3:19-32.

Carnethon MR, Sidney S, Loria CM, et al: Risk factors for the metabolic syndrome. The Coronary Artery Risk Development in Young Adults (CARDIA) study, 1985-2001. Diabetes Care 2004;27:2707-2715.

$\checkmark$ Chen L, Appel LJ, Loria C, et al: Reduction in consumption of sugar-sweetened beverages is associated with weight loss: the PREMIER trial. Am J Clin Nutr 2009;89:12991306.

Chen J, He J, Wildman RP, et al: A randomized controlled trial of dietary fiber intake on serum lipids. Eur J Clin Nutr 2006;60:6268 . 
-Cheng G, Karaolis-Danckert N, Libuda L, et al: Relation of dietary glycemic index, glycemic load, and fiber and whole-grain intakes during puberty to the concurrent development of percent body fat and body mass index. Am J Epidemiol 2009;169:667-677.

-Clarke R, Frost C, Collins R, Appleby P, Peto R: Dietary lipids and blood cholesterol: quantitative meta-analysis of metabolic ward studies. BMJ 1997;314:112-117.

Colditz GA, Manson JE, Stampfer MJ, et al: Diet and risk of clinical diabetes in women. Am J Clin Nutr 1992;55:1018-1023.

Colditz GA, Willett WC, Stampfer MJ, et al: Patterns of weight change and their relation to diet in a cohort of healthy women. Am J Clin Nutr 1990;5:1100-1105.

Connor SL, Gustafson JR, Sexton G, et al: The Diet Habit Survey: a new method of dietary assessment that relates to plasma cholesterol changes. J Am Diet Assoc 1992;92:41-47.

Cust AE, Slimani N, Kaaks R, et al: Dietary carbohydrates, glycemic index, glycemic load, and endometrial cancer risk within the European Prospective Investigation into Cancer and Nutrition cohort. Am J Epidemiol 2007; 166:912-923.

Damiao R, Castro TG, Cardoso MA, et al: Dietary intakes associated with the metabolic syndrome in a cohort of Japanese ancestry. Br J Nutr 2006;96:532-538.

Davis JN, Alexander KE, Ventura EE, et al: Inverse relation between dietary fiber intake and visceral adiposity in overweight Latino youth. Am J Clin Nutr 2009;90:1160-1166.

Davy BM, Melby CL, Beske SD, et al: Oat consumption does not affect resting casual and ambulatory 24-h arterial blood pressure in men with high-normal blood pressure to stage I hypertension. J Nutr 2002;132:394398.

-de Castro TG, Gimeno SG, Ferreira SR, Cardoso MA; and Group, Japanese-Brazilian Diabetes Study: Association of dietary fiber with temporal changes in serum cholesterol in Japanese-Brazilians. J Nutr Sci Vitaminol 2006;52:205-210.

-de Munter JS, Hu FB, Spiegelman D, et al: Whole grain, bran, and germ intake and risk of type 2 diabetes: a prospective cohort study and systematic review. PLoS Med 2007;4:e26.

DGE (Deutsche Gesellschaft für Ernährung, ed): Kohlenhydratzufuhr und Prävention ausgewählter ernährungsmitbedingter Krankheiten - Evidenzbasierte Leitlinie. Bonn 2011a. http://www.dge.de/leitlinie.

DGE (Deutsche Gesellschaft für Ernährung, ed): DGE-Position: Richtwerte für die Energiezufuhr aus Kohlenhydraten und Fett. Ernaehrungs Umschau 2011b;58:152-154.

DGE (Deutsche Gesellschaft für Ernährung, ed): Vollwertig essen und trinken nach den 10 Regeln der DGE. 7. Auflage, Bonn, 2010.

DGE (Deutsche Gesellschaft für Ernährung, ed): Ernährungsbericht 2008. Bonn, 2008.

DGE (Deutsche Gesellschaft für Ernährung, ed): Stellungnahme 'Obst und Gemüse in der Prävention chronischer Krankheiten' Bonn, 2007. http://www.dge.de/pdf/ws/Stellungnahme-OuG-Praevention-chronischer Krankheiten-2007-09-29.pdf (accessed on: September 24, 2010).

DGE (Deutsche Gesellschaft für Ernährung, ed) Fettkonsum und Prävention ausgewählter ernährungsmitbedingter Krankheiten - Evidenzbasierte Leitlinie. Bonn, 2006. http:// www.dge.de/leitlinie.

Dhingra R, Sullivan L, Jacques PF, et al: Soft drink consumption and risk of developing cardiometabolic risk factors and the metabolic syndrome in middle-aged adults in the community. Circulation 2007;116:480-488.

DiMeglio DP, Mattes RD: Liquid versus solid carbohydrate: effects on food intake and body weight. Int J Obes Relat Metab Disord 2000;24:794-800.

Du H, van der A DL, Boshuizen HC, et al: Dietary fiber and subsequent changes in body weight and waist circumference in European men and women. Am J Clin Nutr 2010;91: 329-336.

Du H, van der A DL, van Bakel MM, et al: Dietary glycaemic index, glycaemic load and subsequent changes of weight and waist circumference in European men and women. Int J Obes (Lond) 2009;33:1280-1288.

Dubois L, Farmer A, Girard M, Peterson K: Regular sugar-sweetened beverage consumption between meals increases risk of overweight among preschol-aged children. J Am Diet Assoc 2007; 107:924-934; discussion 934-935.

Eastwood MA, Elton RA, Smith JH: Long-term effect of wholemeal bread on stool weight, transit time, fecal bile acids, fats, and neutral sterols. Am J Clin Nutr 1986;43:343-349.

Ebbeling CB, Feldman HA, Osganian SK, et al: Effects of decreasing sugar-sweetened beverage consumption on body weight in adolescents: a randomized, controlled pilot study. Pediatrics 2006;117:673-680.

Ebbeling CB, Leidig MM, Sinclair KB, et al: Effects of an ad libitum low glycemic load diet on cardiovascular disease risk factors in obese young adults. Am J Clin Nutr 2005;81:976-982.

Eiben G, Lissner L: Health Hunters - an intervention to prevent overweight and obesity in young high-risk women. Int J Obes (Lond) 2006;30:691-696.

Esrey KL, Joseph L, Grover SA: Relationship between dietary intake and coronary heart disease mortality: lipid research clinics prevalence follow-up study. J Clin Epidemiol 1996; 49:211-216.

Feskens EJ, Virtanen SM, Rasanen L, et al: Dietary factors determining diabetes and impaired glucose tolerance. A 20-year followup of the Finnish and Dutch cohorts of the Seven Countries Study. Diabetes Care 1995; 18:1104-1112.

Feskens EJ, Bowles CH, Kromhout D: Carbohydrate intake and body mass index in relation to the risk of glucose intolerance in an elderly population. Am J Clin Nutr 1991;54:136140.
Fiorito LM, Marini M, Francis LA, et al: Beverage intake of girls at age $5 \mathrm{y}$ predicts adiposity and weight status in childhood and adolescence. Am J Clin Nutr 2009;90:935-942.

Fisher E, Boeing H, Fritsche A, et al: Wholegrain consumption and transcription factor7-like 2 (TCF7L2) rs7903146: gene-diet interaction in modulating type 2 diabetes risk. Br J Nutr 2009;101:478-481.

Flint AJ, Hu FB, Glynn RJ, et al: Whole grains and incident hypertension in men. Am J Clin Nutr 2009;90:493-498.

Forman JP, Choi H, Curhan GC: Fructose and vitamin $\mathrm{C}$ intake do not influence risk for developing hypertension. J Am Soc Nephrol 2009;20:863-871.

-Forshee RA, Anderson PA, Storey ML: Sugarsweetened beverages and body mass index in children and adolescents: a meta-analysis. Am J Clin Nutr 2008;87:1662-1671.

Fung TT, Malik V, Rexrode KM, et al: Sweetened beverage consumption and risk of coronary heart disease in women. Am J Clin Nutr 2009;89:1037-1042.

Fung TT, Hu FB, Pereira MA, et al: Whole-grain intake and the risk of type 2 diabetes: a prospective study in men. Am J Clin Nutr 2002; 76:535-540.

Gallus S, Turati F, Tavani A, et al: Soft drinks, sweetened beverages and risk of pancreatic cancer. Cancer Causes Control 2011;22:3339. Epub 2010 Oct 28.

-Garcia-Palmieri MR, Sorlie P, Tillotson J, et al: Relationship of dietary intake to subsequent coronary heart disease incidence: The Puerto Rico Heart Health Program. Am J Clin Nutr 1980;33:1818-1827.

-Garry PJ, Hunt WC, Koehler KM, et al: Longitudinal study of dietary intakes and plasma lipids in healthy elderly men and women. Am J Clin Nutr 1992;55:682-688.

Gatenby SJ, Aaron JI, Jack VA, Mela DJ: Extended use of foods modified in fat and sugar content: nutritional implications in a free-living female population. Am J Clin Nutr 1997;65: 1867-1873.

- George SM, Mayne ST, Leitzmann MF, et al: Dietary glycemic index, glycemic load, and risk of cancer: a prospective cohort study. Am J Epidemiol 2009;169:462-472.

Gibson S: Sugar-sweetened soft drinks and obesity: a systematic review of the evidence from observational studies and interventions. Nutr Res Rev 2008;21:134-147.

Giles GG, Simpson JA, English DR, et al: Dietary carbohydrate, fibre, glycaemic index, glycaemic load and the risk of postmenopausal breast cancer. Int J Cancer 2006;118:18431847.

Gnagnarella P, Gandini S, La Vecchia C, Maisonneuve P: Glycemic index, glycemic load, and cancer risk: a meta-analysis. Am J Clin Nutr 2008;87:1793-1801.

Gordon T, Kagan A, Garcia-Palmieri M, et al: Diet and its relation to coronary heart disease and death in three populations. Circulation 1981;63:500-515. 
-Grandjean AC, Reimers KJ, Bannick KE, Haven MC: The effect of caffeinated, non-caffeinated, caloric and non-caloric beverages on hydration. J Am Coll Nutr 2000;19:591-600.

-Halkjaer J, Tjonneland A, Thomsen BL, et al: Intake of macronutrients as predictors of $5-y$ changes in waist circumference. Am J Clin Nutr 2006;84:789-797.

-Halkjaer J, Sorensen TI, Tjonneland A, et al: Food and drinking patterns as predictors of 6 -year BMI-adjusted changes in waist circumference. Br J Nutr 2004;92:735-748.

Halton TL, Liu S, Manson JE, Hu FB: Low-carbohydrate-diet score and risk of type 2 diabetes in women. Am J Clin Nutr 2008;87: 339-346.

Halton TL, Willett WC, Liu S, et al: Low-carbohydrate-diet score and the risk of coronary heart disease in women. N Engl J Med 2006; 355:1991-2002.

-Hare-Bruun H, Flint A, Heitmann BL: Glycemic index and glycemic load in relation to changes in body weight, body fat distribution, and body composition in adult Danes. Am J Clin Nutr 2006;84:871-879.

Harding AH, Day NE, Khaw KT, et al: Dietary fat and the risk of clinical type 2 diabetes: the European prospective investigation of Cancer-Norfolk study. Am J Epidemiol 2004;159: 73-82.

Haub MD, Simons TR, Cook CM, et al: Calciumfortified beverage supplementation on body conposition in postmenopausal women. Nutr J 2005;4:21.

Heaton KW, Manning AP, Hartog M: Lack of effect on blood lipid and calcium concentrations of young men on changing from white to wholemeal bread. Br J Nutr 1976;35:55-60.

Heinen MM, Verhage BA, Lumey L, et al: Glycemic load, glycemic index, and pancreatic cancer risk in the Netherlands Cohort Study. Am J Clin Nutr 2008;87:970-977.

- Hodge AM, English DR, O’Dea K, Giles GG: Glycemic index and dietary fiber and the risk of type 2 diabetes. Diabetes Care 2004;27: 2701-2706.

Hopping BN, Erber E, Grandinetti A, et al: Dietary fiber, magnesium, and glycemic load alter risk of type 2 diabetes in a multiethnic cohort in Hawaii. J Nutr 2010;140:68-74.

Howard BV, Manson JE, Stefanick ML, et al: Lowfat dietary pattern and weight change over 7 years: the Women's Health Initiative Dietary Modification Trial. JAMA 2006;295:39-49.

- Howarth NC, Murphy SP, Wilkens LR, et al: The association of glycemic load and carbohydrate intake with colorectal cancer risk in the Multiethnic Cohort Study. Am J Clin Nutr 2008;88:1074-1082.

Hu FB, Manson JE, Stampfer MJ, et al: Diet, Lifestyle, and the risk of type 2 diabetes mellitus in women. N Engl J Med 2001;345:790-797.

-Huttunen JK, Mäkinen KK, Scheinin A: Turku sugar studies XI. Effects of sucrose, fructose and xylitol diets on glucose, Lipid and urate metabolism. Acta Odontol Scand 1976;34: 345-351.
International Journal of Obesity: 9th and 10th international symposia organized by the Merck Frosst/CIHR Research Chair in Obesity, Université Laval, Québec, Qué, Canada, Auberge Saint-Antoine, Québec, QC, Canada, 15-16 November 2007. 1. Gastrointestinal Tract, Obesity and Diabetes, 2. Childhood Obesity: Biological/Environmental Determinants and Preventative Strategies 2009;33:S1.

Iqbal Kring N, Heitmann BL: Fiber intake, not dietary energy density, is associated with subsequent change in BMI $\mathrm{z}$-score among sub-groups of children. Obes Facts 2008; 1 : 331-338.

-Iqbal SI, Helge JW, Heitmann BL: Do energy density and dietary fiber influence subsequent 5 -year weight changes in adult men and women? Obesity (Silver Spring) 2006;14: 106-114.

-Jakobsen MU, O'Reilly EJ, Heitmann BL, et al: Major types of dietary fat and risk of coronary heart disease: a pooled analysis of $11 \mathrm{co}-$ hort studies. Am J Clin Nutr 2009;89:14251432 .

-James J, Thomas P, Kerr D: Preventing childhood obesity: two year follow-up results fron the Christchurch obesity prevention programme in schools (CHOPPS). BMJ 2007; 335:762.

James J, Thomas P, Cavan D, Kerr D: Preventing childhood obesity by reducing consumption of carbonated drinks: cluster randomised controlled trial. BMJ 2004;328:1237.

Janket SJ, Manson JE, Sesso H, et al: A prospective study of sugar intake and risk of type 2 diabetes in women. Diabetes Care 2003;26: 1008-1015.

Jiao L, Flood A, Subar AF, et al: Glycemic index, carbohydrates, glycemic load, and the risk of pancreatic cancer in a prospective cohort study. Cancer Epidemiol Biomarkers Prev 2009;18:1144-1151.

Johnson L, Mander AP, Jones LR, et al: Is sugarsweetened beverage consumption associated with increased fatness in children? Nutrition 2007;23:557-563.

Jones JM: Dietary sweeteners containing fructose: overview of a workshop on the state of the science. J Nutr 2009;139:1210S-1213S.

Kabat GC, Shikany JM, Beresford SA, et al: Dietary carbohydrate, glycemic index, and glycemic load in relation to colorectal cancer risk in the Women's Health Initiative. Cancer Causes Control 2008;19:1291-1298.

- Kaitosaari T, Rönnemaa T, Raitakari O, et al: Effect of 7-year infancy-onset dietary intervention on serum lipoproteins and lipoprotein subclasses in healthy children in the prospective, randomized Special Turku Coronary Risk Factor Intervention Project for children (STRIP) Study. Circulation 2003; 108:672-677.

Karnehed N, Tynelius P, Heitmann BL, Rasmussen F: Physical activity, diet and gene-environment interactions in relation to body mass index and waist circumference: the
Swedish young male twins study. Public Health Nutr 2006;9:851-858.

Kasim SE, Martino S, Kim PN, et al: Dietary and anthropometric determinants of plasma lipoproteins during a long-term low-fat diet in healthy women. Am J Clin Nutr 1993;57: 146-153.

-Kasim-Karakas SE, Almario RU, Mueller WM, Peerson J: Changes in plasma lipoproteins during low-fat, high-carbohydrate diets: effects of energy intake. Am J Clin Nutr 2000; 71:1439-1447.

Kelly SAM, Summerbell CD, Brynes A, et al: Wholegrain cereals for coronary heart disease. Cochrane Database of Systematic Reviews 2007, Issue 2. Art. No: CD005051.

Kelly S, Frost G, Whittaker V, et al: Low glycaemic index diets for coronary heart disease. Cochrane Database of Systematic Reviews 2004, Issue 4. Art. No: CD004467.

-Klesges RC, Isbell TR, Klesges LM: Relationship between dietary restraint, energy intake, physical activity, and body weight: a prospective analysis. J Abnorm Psychol 1992;10: 668-674.

Knorpp L, Kroke A: Evidenzbasierung von Massnahmen der bevölkerungsbezogenen Prävention von ernährungsassoziierten Erkrankungen. Ernaehrungs Umschau 2011; 58:73-79.

Kochar J, Djousse L, Gaziano JM: Breakfast cereals and risk of type 2 diabetes in the Physicians' Health Study I. Obesity (Silver Spring) 2007;15:3039-3044.

Koh-Banerjee P, Franz M, Sampson L, et al: Changes in whole-grain, bran, and cereal fiber consumption in relation to 8 -y weight gain among men. Am J Clin Nutr 2004;80: 1237-1245.

Kral TV, Stunkard AJ, Berkowitz RI, et al: Beverage consumption patterns of children born at different risk of obesity. Obesity (Silver Spring) 2008;16:1802-1808.

Krauss RM, Blanche PJ, Rawlings RS, et al: Separate effects of reduced carbohydrate intake and weight loss on atherogenic dyslipidemia. Am J Clin Nutr 2006;83:1025-1031.

Krishnan S, Rosenberg L, Singer M, et al: Glycemic index, glycemic load, and cereal fiber intake and risk of type 2 diabetes in US black women. Arch Intern Med 2007;167:2304-2309.

Kroke A, Boeing H, Rossnagel K, Willich SN: History of the concept of 'levels of evidence' and their current status in relation to primary prevention through lifestyle interventions. Public Health Nutr 2003;2: 279-284.

Kvaavik E, Andersen LF, Klepp KI: The stability of soft drinks intake from adolescence to adult age and the association between longterm consumption of soft drinks and lifestyle factors and body weight. Public Health Nutr 2005;8:149-157.

Lagiou P, Sandin S, Weiderpass E, et al: Low carbohydrate-high protein diet and mortality in a cohort of Swedish women. J Intern Med 2007;261:366-374. 
Lagström H, Seppänen R, Jokinen E, et al: Influence of dietary fat on the nutrient intake and growth of children from 1 to $5 \mathrm{y}$ of age: the Special Turku Coronary Risk Factor Intervention Project. Am J Clin Nutr 1999;69: 516-523.

Lajous M, Boutron-Ruault MC, Fabre A, et al: Carbohydrate intake, glycemic index, glycemic load, and risk of postmenopausal breast cancer in a prospective study of French women. Am J Clin Nutr 2008;87:1384-1391.

-Larsson SC, Bergkvist L, Wolk A: Glycemic load, glycemic index and breast cancer risk in a prospective cohort of Swedish women. Int J Cancer 2009;125:153-157.

-Larsson SC, Giovannucci E, Wolk A: Dietary carbohydrate, glycemic index, and glycemic load in relation to risk of colorectal cancer in women. Am J Epidemiol 2007;165:256-261.

- Larsson SC, Bergkvist L, Wolk A: Glycemic load, glycemic index and carbohydrate intake in relation to risk of stomach cancer: a prospective study. Int J Cancer 2006a;118:3167-3169.

-Larsson SC, Bergkvist L, Wolk A: Consumption of sugar and sugar-sweetened foods and the risk of pancreatic cancer in a prospective study. Am J Clin Nutr 2006b;84:1171-1176.

Laurson K, Eisenmann JC, Moore S: Lack of association between television viewing, soft drinks, physical activity and body mass index in children. Acta Paediatr 2008;97:795800.

Lee YP, Mori TA, Puddey IB, et al: Effects of lupin kernel flour-enriched bread on blood pressure: a controlled intervention study. Am J Clin Nutr 2009;89:766-772

Lee-Han H, Cousins M, Beaton M, et al: Compliance in a randomized clinical trial of dietary fat reduction in patients with breast dysplasia. Am J Clin Nutr 1988;48:575-586.

- Levitan EB, Mittleman MA, Håkansson N, Wolk A: Dietary glycemic index, dietary glycemic load, and cardiovascular disease in middleaged and older Swedish men. Am J Clin Nutr 2007;85:1521-1526.

- Libuda L, Alexy U, Sichert-Hellert, et al: Pattern of beverage consumption and long term association with the body weight status in German adolescents - results from the DONALD study. Br J Nutr 2008;99:1370-1379.

Lim S, Zoellner JM, Lee JM, et al: Obesity and sugar-sweetened beverages in African-American preschool children: a longitudinal study. Obesity (Silver Spring) 2009;17:1262-1268.

- Lin YC, Lyle RM, McCabe LD, et al: Dairy calcium is related to changes in body composition during a two-year exercise intervention in young women. J Am Coll Nutr 2000;19:754-760.

Lindstrom J, Peltonen M, Eriksson JG, et al: High-fibre, low-fat diet predicts long-term weight loss and decreased type 2 diabetes risk: the Finnish Diabetes Prevention Study. Diabetologia 2006;49:912-920.

Liu S, Chou EL: Dietary glycemic load and type 2 diabetes: modeling the glucose-raising potential of carbohydrates for prevention. Am J Clin Nutr 2010;92:675-677.
Liu S, Willett WC, Manson JE, et al: Relation between changes in intakes of dietary fiber and grain products and changes in weight and development of obesity among middle-aged women. Am J Clin Nutr 2003;78:920-927.

Liu S, Manson JE, Stampfer MJ, et al: A prospective study of whole-grain intake and risk of type 2 diabetes mellitus in US women. Am J Public Health 2000a;90:1409-1415.

Liu S, Willett WC, Stampfer MJ, et al: A prospective study of dietary glycemic load, carbohydrate intake, and risk of coronary heart disease in US women. Am J Clin Nutr 2000b;71: $1455-1461$.

Livesey G, Taylor R: Fructose consumption and consequences for glycation, plasma triacylglycerol, and body weight: meta-analyses and meta-regression models of intervention studies. Am J Clin Nutr 2008;88:1419-1437.

Livesey G, Taylor R, Hulshof T, Howlett J: Glycemic response and health - a systematic review and meta-analysis: relations between dietary glycemic properties and health outcomes. Am J Clin Nutr 2008;87:258S-268S.

Lock S, Ford MA, Bagley R, Green LF: The effect on plasma lipids of the isoenergetic replacement of table sucrose by dried glucose syrup (maize-syrup solids) in the normal diet of adult men over a period of 1 year. Br J Nutr 1980;43:251-256.

Ludwig DS, Peterson KE, Gortmaker SL: Relation between consumption of sugar-sweetened drinks and childhood obesity: a prospective, observational analysis. Lancet 2001;357:505-508.

- Ludwig DS, Pereira MA, Kroenke CH, et al: Dietary fiber, weight gain, and cardiovascular disease risk factors in young adults. JAMA 1999;282:1539-1546.

-Lundgren H, Bengtsson C, Blohme G, et al: Dietary habits and incidence of noninsulin-dependent diabetes mellitus in a population study of women in Gothenburg, Sweden. Am J Clin Nutr 1989;49:708-712.

Lutsey PL, Steffen LM, Stevens J: Dietary intake and the development of the metabolic syndrome: The Atherosclerosis Risk in Communities Study. Circulation 2008;117:754-761.

Ma Y, Li Y, Chiriboga DE, et al: Association between carbohydrate intake and serum lipids. J Am Coll Nutr 2006;25:155-163.

- Ma Y, Olendzki B, Chiriboga D, et al: Association between dietary carbohydrates and body weight. Am J Epidemiol 2005;161:359-367.

Mack I, Hauner H: Low Carb - kohlenhydratarme Kostformen unter die Lupe genommen. Ernaehrungs Umschau 2007;54: 720-726.

-Magarey AM, Daniels LA, Boulton TJ, Cockington RA: Does fat intake predict adiposity in healthy children and adolescents aged 2-15 years? A longitudinal analysis. Eur J Clin Nutr 2001;55:471-481.

Malik VS, Popkin BM, Bray GA, et al: Sugarsweetened beverages and risk of metabolic syndrome and type 2 diabetes: a meta-analysis. Diabetes Care 2010;33:2477-2483.
Malik VS, Willett WC, Hu FB: Sugar-sweetened beverages and BMI in children and adolescents: reanalyses of a meta-analysis. Am J Clin Nutr 2009;89:438-439.

-Marett R, Slavin JL: No long-term benefits of supplementation with arabinogalactan on serum lipids and glucose. J Am Diet Assoc 2004;104:636-639.

- Marshall JA, Hoag S, Shetterly S, Hamman RF: Dietary fat predicts conversion from impaired glucose tolerance to NIDDM. The San Luis Valley Diabetes Study. Diabetes Care 1994;17:50-56.

Mattes RD, Shikany JM, Kaiser KA, Allison DB: Nutritively sweented beverage consumption and body weight: a systematic review and meta-analysis of randomized experiments. Obes Rev 2010; [Epub ahead of print].

McGee DL, Reed DM, Yano K, et al: Ten-year incidence of coronary heart disease in the Honolulu Heart Program. Relationship to nutrient intake. Am J Epidemiol 1984;119: 667-676.

$\rightarrow$ McMillen-Price J, Petocz P, Atkinson F, et al: Comparison of 4 diets of varying glycemic load on weight loss and cardiovascular risk reduction in overweight and obese young adults: a randomized controlled trial. Arch Intern Med 2006;166:1466-1475.

-Mellen PB, Walsh T, Herrington D: Whole grain intake and cardiovascular disease: A metaanalysis. Nutr Metab Cardiovasc Dis 2008; 18:283-290.

Mendez MA, Pera G, Agudo A, et al: Cereal fiber intake may reduce risk of gastric adenocarcinomas: the EPIC-EURGAST study. Int J Cancer 2007;121:1618-1623.

Mensink RP, Zock PL, Kester AD, Katan MB: Effects of dietary fatty acids and carbohydrates on the ratio of serum total to HDL cholesterol and on serum lipids and apolipoproteins: a meta-analysis of 60 controlled trials. Am J Clin Nutr 2003;77:1146-1155.

$\rightarrow$ Mensink RP, Katan MB: Effect of dietary fatty acids on serum lipids and lipoproteins - a meta-analysis of 27 trials. Arteriosclerosis and Thrombosis 1992;12:911-919.

Mente A, de Koning L, Shannon HS, Anand SS: A systematic review of the evidence supporting a causal link between dietary factors and coronary heart disease. Arch Intern Med 2009;169:659-669.

- Meyer KA, Kushi LH, Jacobs DR Jr, et al: Carbohydrates, dietary fiber, and incident type 2 diabetes in older women. Am J Clin Nutr 2000;71:921-930.

Millen BE, Pencina MJ, Kimokoti RW, et al: Nutritional risk and the metabolic syndrome in women: opportunities for preventive intervention from the Framingham Nutrition. Am J Clin Nutr 2006;84:434-441.

Mirmiran P, Noori N, Azizi F: A prospective study of determinants of the metabolic syndrome in adults. Nutr Metab Cardiovasc Dis 2008; 18:567-573 
Monterrosa AE, Haffner SM, Stern MP, Hazuda HP: Sex difference in lifestyle factors predictive of diabetes in Mexican-Americans. Diabetes Care 1995; 18:448-456.

Montonen J, Jarvinen R, Knekt P, et al: Consumption of sweetened beverages and intakes of fructose and glucose predict type 2 diabetes occurrence. J Nutr 2007;137:1447-1454.

Montonen J, Knekt P, Jarvinen R, et al: Wholegrain and fiber intake and the incidence of type 2 diabetes. Am J Clin Nutr 2003;77:622629.

Mosdol A, Witte DR, Frost G, et al: Dietary glycemic index and glycemic load are associated with high-density-lipoprotein cholesterol at baseline but not with increased risk of diabetes in the Whitehall II study. Am J Clin Nutr 2007;86:988-994.

Mozaffarian D, Micha R, Wallace S: Effects on coronary heart disease of increasing polyunsaturated fat in place of saturated fat: a systematic review and meta-analysis of randomized controlled trials. PLoS Med 2010; 7:e1000252.

-Mozaffarian D, Kumanyika SK, Lemaitre RN, et al: Cereal, fruit, and vegetable fiber intake and the risk of cardiovascular disease in elderly individuals. JAMA 2003;289:1659-1666.

-Mrdjenovic G, Levitsky DA: Nutritional and energetic consequences of sweetened drink consumption in 6- to 13-year-old children. J Pediatr 2003;142:604-610.

MRI (Max Rubner-Institut): Nationale Verzehrsstudie II. Ergebnissbericht Teil 1. Karlsruhe, 2008;www.was-esse-ich.de/uploads/media/NVSII_Abschlussbericht_ Teil_1.pdf (accessed on: April 13, 2010).

-Mueller NT, Odegaard A, Anderson K, et al: Soft drink and juice consumption and risk of pancreatic cancer: the Singapore Chinese Health Study. Cancer Epidemiol Biomarkers Prev 2010;19:447-455.

- Mulholland HG, Murray LJ, Cardwell CR, Cantwell MM: Glycemic index, glycemic loas, and risk of digestive tract neoplasm: a systematic review and meta-analysis. Am J Clin Nutr 2009;89:568-576.

Mulholland HG, Murray LJ, Cardwell CR, Cantwell MM: Dietary glycaemic index, glycaemic load and breast cancer risk: a systematic review and meta-analysis. Br J Cancer 2008a;99:1170-1175.

-Mulholland HG, Murray LJ, Cardwell CR, Cantwell MM: Dietary glycaemic index, glycaemic load and endometrial and ovarian cancer risk: a systematic review and metaanalysis. Br J Cancer 2008b;99:434-441.

Mundt CA, Baxter-Jones AD, Whiting SJ, et al: Relationships of activity and sugar drink intake on fat mass development in youths. Med Sci Sports Exerc 2006;38:1245-1254.

Munoz D: The efficacy of two brief interventions to reduce soda consumption in a college population (Dissertation). State University of New York: Albany, NY, 2006.

-National Cholesterol Education Program (NCEP) Expert Panel on Detection, Evalua- tion, and Treatment of High Blood Cholesterol in Adults (Adult Treatment Panel III): Executive summary of the third report. JAMA 2001;285:2486-2497.

Nettleton JA, Lutsey PL, Wang Y, et al: Diet soda intake and risk of incident metabolic syndrome and type 2 diabetes in the Multi-Ethnic Study of Atherosclerosis (MESA). Diabetes Care 2009;32:688-694.

- Newby PK, Peterson KE, Berkey CS, et al: Beverage consumption is not associated with changes in weight and body mass index among lowincome preschool children in North Dakota. J Am Diet Assoc 2004;104:1086-1094.

Nicklas TA, Dwyer J, Feldman HA, et al: Serum cholesterol levels in children are associated with dietary fat and fatty acid intake. J Am Diet Assoc 2002; 102:511-517.

- Nielsen TG, Olsen A, Christensen J, et al: Dietary carbohydrate intake is not associated with the breast cancer incidence rate ratio in postmenopausal Danish women. J Nutr 2005; 135:124-128.

Niinikoski H, Lagström H, Jokinen E, et al: Impact of repeated dietary counseling between infancy and 14 years of age on dietary intakes and serum lipids and lipoproteins: the STRIP Study. Circulation 2007;116:1032-1040.

- Nissinen K, Mikkilä V, Männistö S, et al: Sweets and sugar-sweetened soft drink intake in childhood in relation to adult BMI and overweight. The Cardiovascular Risk in Young Finns Study. Public Health Nutr 2009;12: 2018-2026.

Nöthlings U, Murphy SP, Wilkens LR, et al: Dietary glycemic load, added sugars, and carbohydrates as risk factors for pancreatic cancer: the Multiethnic Cohort Study. Am J Clin Nutr 2007;86:1495-1501.

Nomura AM, Wilkens LR, Murphy SP, et al: Association of vegetable, fruit, and grain intakes with colorectal cancer: the Multiethnic Cohort Study. Am J Clin Nutr 2008;88:730-737.

Nomura AM, Hankin JH, Henderson BE, et al: Dietary fiber and colorectal cancer risk: the multiethnic cohort study. Cancer Causes Control 2007; 18:753-764.

Nooyens AC, Visscher TL, Schuit AJ, et al: Effects of retirement on lifestyle in relation to changes in weight and waist circumference in Dutch men: a prospective study. Public Health Nutr 2005;8:1266-1274.

-Otani T, Iwasaki M, Ishihara J, et al: Dietary fibre intake and subsequent risk of colorectal cancer: the Japan Public Health Center-based prospective study. Int J Cancer 2006;119: $1475-1480$.

- Oxlund AL, Heitmann BL: Glycaemic index and glycaemic load in relation to blood lipids -6 years of follow-up in adult Danish men and women. Public Health Nutr 2006;9:737-745.

Palmer JR, Boggs DA, Krishnan S, et al: Sugarsweetened beverages and incidence of type 2 diabetes mellitus in African American women. Arch Intern Med 2008;168:1487-1492.

- Park Y, Brinton LA, Subar AF, Hollenbeck A, Schatzkin A: Dietary fiber intake and risk of breast cancer in postmenopausal women: the National Institutes of Health-AARP Diet and Health Study. Am J Clin Nutr 2009;90: 664-671.

Park Y, Hunter DJ, Spiegelman D, et al: Dietary fiber intake and risk of colorectal cancer: a pooled analysis of prospective cohort studies. JAMA 2005;294:2849-2857.

- Parker DR, Gonzalez S, Derby CA, et al: Dietary factors in relation to weight change among men and women from two southeastern New England communities. Int $J$ Obes Relat Metab Disord 1997;21:103-109.

- Patel AV, McCullough ML, Pavluck AL, et al: Glycemic load, glycemic index, and carbohydrate intake in relation to pancreatic cancer risk in a large US cohort. Cancer Causes Control 2007; 18:287-294.

- Paynter NP, Yeh HC, Voutilainen S, et al: Coffee and sweetened beverage consumption and the risk of type 2 diabetes mellitus: the atherosclerosis risk in communities study. Am J Epidemiol 2006;164:1075-1084.

- Pereira MA, O'Reilly E, Augustsson K, et al: Dietary fiber and risk of coronary heart disease: a pooled analysis of cohort studies. Arch Intern Med 2004;164:370-376.

- Philippou E, Bovill-Taylor C, Rajkumar C, et al: Preliminary report: the effect of a 6-month dietary glycemic index manipulation in addition to healthy eating advice and weight loss on arterial compliance and 24-hour ambulatory blood pressure in men: a pilot study. Metabolism 2009;58:1703-1708.

- Phillips SM, Bandini LG, Naumova EN, et al: Energy-dense snack food intake in adolescence: longitudinal relationship to weight and fatness. Obes Res 2004;12:461-472.

Priebe MG, van Binsbergen JJ, de Vos R, Vonk $\mathrm{RJ}$ : Whole grain foods for the prevention of type 2 dibetes mellitus. Cochrane Database of Systematic Reviews 2008; Issue 1. Art.No: CD006061.

-Raben A, Vasilaras TH, Moller AC, Astrup A: Sucrose compared with artificial sweeteners: different effects on ad libitum food intake and body weight after 10 weeks of supplementation in overweight subjects. Am J Clin Nutr 2002;76:721-729.

Rock CL, Flatt SW, Thomson CA, et al; for the Women's Healthy Eating and Living (WHEL) Study Group: Plasma triacylglycerol and HDL cholesterol concentrations confirm self-reported changes in carbohydrate and fat intakes in women in a diet intervention trial. J Nutr 2004;134:342-347.

- Rodearmel SJ, Wyatt HR, Stroebele N, et al: Small changes in dietary sugar and physical activity as an approach to preventing excessive weight gain: the America on the Move family study. Pediatrics 2007;120:869-879.

-Rolland-Cachera MF, Deheeger M, Akrout M, Bellisle F: Influence of macronutrients on adiposity development: a follow up study of nutrition and growth from 10 months to 8 years of age. Int J Obes Relat Metab Disord 1995;19:573-578. 
-Ruottinen S, Rönnemaa T, Niinikoski H, et al: Carbohydrate intake, serum lipids and apolipoprotein E phenotype show association in children. Acta Paediatrica 2009;98:1667-1673.

-Ruottinen S, Niinikoski H, Lagström H, et al: High sucrose intake is associated with poor quality of diet and growth between 13 months and 9 years of age: The Special Turku Coronary Risk Factor Intervention Project. Pediatrics 2008;121:e1676-e1685.

- Sacks FM, Handysides GH, Marais GE, et al: Effects of a low-fat diet on plasma lipoprotein levels. Arch Intern Med 1986;146:1573-1577.

- Sahyoun NR, Anderson AL, Tylavsky FA, et al: Dietary glycemic index and glycemic load and the risk of type 2 diabetes in older adults. Am J Clin Nutr 2008;87:126-131.

- Sahyoun NR, Jacques PF, Zhang XL, et al: Whole-grain intake is inversely associated with the metabolic syndrome and mortality in older adults. Am J Clin Nutr 2006;83:124131.

- Salmeron J, Hu FB, Manson JE, et al: Dietary fat intake and risk of type 2 diabetes in women. Am J Clin Nutr 2001;73:1019-1026.

- Salmeron J, Ascherio A, Rimm EB, et al: Dietary fiber, glycemic load, and risk of NIDDM in men. Diabetes Care 1997a;20:545-550.

- Salmeron J, Manson JE, Stampfer MJ, et al: Dietary fiber, glycemic load, and risk of noninsulin-dependent diabetes mellitus in women. Jama 1997b;277:472-477.

- Sandström B, Marckmann P, Bindslev N: An eight-month controlled study of a low-fat high-fibre diet: effects on blood lipids and blood pressure in healthy young subjects. Eur J Clin Nutr 1992;46:95-109.

- Saris WH, Astrup A, Prentice AM, et al: Randomized controlled trial of changes in dietary carbohydrate/fat ratio and simple vs complex carbohydrates on body weight and blood lipids: the CARMEN study. The Carbohydrate Ratio Management in European National diets. Int J Obes Relat Metab Disord 2000;24:1310-1318.

-Schatzkin A, Mouw T, Park Y, et al: Dietary fiber and whole-grain consumption in relation to colorectal cancer in the NIH-AARP Diet and Health Study. Am J Clin Nutr 2007;85:13531360.

Schulz M, Liese AD, Fang F, et al: Is the association between dietary glycemic index and type 2 diabetes modified by waist circumference? Diabetes Care 2006;29:1102-1104.

-Schulze MB, Schulz M, Heidemann C, et al: Carbohydrate intake and incidence of type 2 diabetes in the European Prospective Investigation into Cancer and Nutrition (EPIC)Potsdam Study. Br J Nutr 2008;99:1107-1116.

- Schulze MB, Hoffmann K, Boeing H, et al: An accurate risk score based on anthropometric, dietary, and lifestyle factors to predict the development of type 2 diabetes. Diabetes Care 2007a;30:510-515.

-Schulze MB, Schulz M, Heidemann C, et al: Fiber and magnesium intake and incidence of type 2 diabetes: a prospective study and me- ta-analysis. Arch Intern Med 2007b;167: 956-965.

-Schulze MB, Liu S, Rimm EB, et al: Glycemic index, glycemic load, and dietary fiber intake and incidence of type 2 diabetes in younger and middle-aged women. Am J Clin Nutr 2004a;80:348-356.

Schulze MB, Manson JE, Ludwig DS, et al: Sugar-sweetened beverages, weight gain, and incidence of type 2 diabetes in young and middle-aged women. Jama 2004b;292:927-934.

Shah M, Adams-Huet B, Garg A: Effect of highcarbohydrate or high-cis-monounsaturated fat diets on blood pressure: a meta-analysis of intervention trials. Am J Clin Nutr 2007; 85:1251-1256.

-Shin A, Li H, Shu XO, et al: Dietary intake of calcium, fiber and other micronutrients in relation to colorectal cancer risk: Results from the Shanghai Women's Health Study. Int J Cancer 2006;119:2938-2942.

- Sichieri R, Paula Trotte A, de Souza RA, Veiga GV: School randomised trial on prevention of excessive weight gain by discouraging students from drinking sodas. Public Health Nutr 2009;12:197-202.

- Sichieri R, Moura AS, Genelhu V, et al: An 18-mo randomized trial of a low-glycemic-index diet and weight change in Brazilian women. Am J Clin Nutr 2007;86:707-713.

-Siewert JR, Ott K: Are squamous and adenocarcinomas of the esophagus the same disease? Semin Radiat Oncol 2007;17:38-44.

-Simons-Morton DG, Hunsberger SA, Van Horn $\mathrm{L}$, et al: Nutrient intake and blood pressure in the Dietary Intervention Study in Children. Hypertension 1997;29:930-936.

- Siri-Tarino PW, Sun Q, Hu FB, Krauss RM: Meta-analysis of prospective cohort studies evaluating the association of saturated fat with cardiovascular disease. Am J Clin Nutr 2010;91:535-546.

-Skinner JD, Bounds W, Carruth BR, et al: Predictors of children's body mass index: a longitudinal study of diet and growth in children aged 2-8 years. Int J Obes Relat Metab Disord 2004;28:476-482.

-Skinner JD, Bounds W, Carruth BR, Ziegler P: Longitudinal calcium intake is negatively related to children's body fat indexes. J Am Diet Assoc 2003;103:1626-1631.

-Sloth B, Krog-Mikkelsen I, Flint A, et al: No difference in body weight decrease between a lowglycemic-index and a high-glycemic-index diet but reduced LDL cholesterol after 10-weeks ad libitum intake of the low-glycemic-index diet. Am J Clin Nutr 2004;80:337-347.

- Sluijs I, van der Schouw YT, van der AD, et al: Carbohydrate quantity and quality and risk of type 2 diabetes in the European Prospective Investigation into Cancer and Nutrition-Netherlands (EPIC-NL) study. Am J Clin Nutr 2010;92:905-911.

-Stamler J, Liu K, Ruth KJ, et al: Eight-year blood pressure change in middle-aged men: relationship to multiple nutrients. Hypertension 2002;39:1000-1006.
Stamler J, Caggiula AW, Grandits GA: Relation of body mass and alcohol, nutrient, fiber, and caffeine intakes to blood pressure in the special intervention and usual care groups in the Multiple Risk Factor Intervention Trial. Am J Clin Nutr 1997;65(1 suppl):338-365.

-Stanhope KL, Schwarz JM, Keim NL, et al: Consuming fructose-sweetened, not glucosesweetened, beverages increases visceral adiposity and lipids and decreases insulin sensitivity in overweight/obese humans. J Clin Invest 2009;119:1322-1334.

- Steffen LM, Kroenke CH, Yu X, et al: Associations of plant food, dairy product, and meat intakes with 15-years incidence of elevated blood pressure in young black and white adults: the Coronary Artery Risk Development in Young Adults (CARDIA) Study. Am J Clin Nutr 2005;82:1169-1177.

Stehle P, Oberritter H, Büning-Fesel M, Heseker $\mathrm{H}$ : Grafische Umsetzung von Ernährungsrichtlinien - traditionelle und neue Ansätze. Ernaehrungs Umschau 2005;52:128-135.

- Stevens J, Ahn K, Juhaeri, et al: Dietary fiber intake and glycemic index and incidence of diabetes in African-American and white adults: the ARIC study. Diabetes Care 2002; 25:1715-1721.

- Strayer L, Jacobs DR Jr, Schairer C, et al: Dietary carbohydrate, glycemic index, and glycemic load and the risk of colorectal cancer in the BCDDP cohort. Cancer Causes Control 2007; 18:853-863

- Streppel MT, Ocké MC, Boshuizen HC, et al: Dietary fiber intake in relation to coronary heart disease and all-cause mortality over 40 years: the Zutphen Study. Am J Clin Nutr 2008;88:1119-1125.

-Streppel MT, Arends LR, van 't Veer P, et al: Dietary fiber and blood pressure: a meta-analysis of randomized placebo-controlled trials. Arch Intern Med 2005;165:150-156.

Striegel-Moore RH, Thompson D, Affenito SG, et al: Correlates of beverage intake in adolescent girls: the National Heart, Lung, and Blood Institute Growth and Health Study. J Pediatr 2006;148:183-187.

Suzuki R, Rylander-Rudqvist T, Ye W, et al: Dietary fiber intake and risk of postmenopausal breast cancer defined by estrogen and progesterone receptor status - a prospective cohort study among Swedish women. Int J Cancer 2008;122:403-412.

Swarbrick MM, Stanhope KL, Elliot SS, et al: Consumption of fructose-sweetened beverages for 10 weeks increases postprandial triacylglycerol and apolipoprotein-B concentrations in overweight and obese women. $\mathrm{Br}$ J Nutr 2008;100:947-952.

Tai ES, Fok AC, Chu R, Tan CE: A study to assess the effect of dietary supplementation with soluble fibre (Minolest) on lipid levels in normal subjects with hypercholesterolaemia. Ann Acad Med Singapore 1999;28:209-213.

-Talati R, Baker WL, Pabilonia MS, et al: The effects of barley-derived soluble fiber on serum lipids. Ann Fam Med 2009;7:157-163. 
-Tam CS, Garnett SP, Cowell CT, et al: Soft drink consumption and excess weight gain in Australian school students: results from the $\mathrm{Ne}$ pean study. Int J Obes (Lond) 2006;30:10911093.

-Tappy L, Le K-A: Metabolic effects of fructose and the worldwide increase in obesity. Physiol Rev 2010;90:23-46.

Thomas DE, Elliott EJ, Baur L: Low glycaemic index or low glycaemic load diets for overweight and obesity (Review). Cochrane Database of Systematic Reviews 2007; Issue 3. Art. No: CD005105.

Tillotson JL, Grandits GA, Bartsch GE, Stamler J: Relation of dietary carbohydrates to blood lipids in the special intervention and usual care groups in the Multiple Risk Factor Intervention. Am J Clin Nutr 1997a;65:314-326.

Tillotson JL, Grandits GA, Bartsch GE, Stamler J: Relation of dietary fiber to blood lipids in the special intervention and usual care groups in the Multiple Risk Factor Intervention Trial. Am J Clin Nutr 1997b;65:327-337.

-Tinker LF, Bonds DE, Margolis KL, et al: Low-fat dietary pattern and risk of treated diabetes mellitus in postmenopausal women: the Women's Health Initiative randomized controlled dietary modification trial. Arch Intern Med 2008;168:1500-1511.

Tordoff MG, Alleva AM: Effect of drinking soda sweetened with aspartame or high-fructose corn syrup on food intake and body weight. Am J Clin Nutr 1990;51:963-969.

-Tucker LA, Thomas KS: Increasing total fiber intake reduces risk of weight and fat gains in women. J Nutr 2009;139:576-581.

-Twisk JW, Kemper HC, van Mechelen W, et al: Body fatness: longitudinal relationship of body mass index and the sum of skinfolds with other risk factors for coronary heart disease. Int J Obes Relat Metab Disord 1998; 22:915-922.

Twisk JW, Kemper HC, van Mechelen W, Post GB: Which lifestyle parameters discriminate high- from low-risk participants for coronary heart disease risk factors. Longitudinal analysis covering adolescence and young adulthood. J Cardiovasc Risk 1997;4:393400 .

van Bakel MM, Kaaks R, Feskens EJ, et al: Dietary glycaemic index and glycaemic load in the European Prospective Investigation into Cancer and Nutrition. Eur J Clin Nutr 2009; 63:S188-S205.

van Dam RM, Seidell JC: Carbohydrate intake and obesity. Eur J Clin Nutr 2007;61(suppl 1):75-99. van Dam RM, Rimm EB, Willett WC, et al: Dietary patterns and risk for type 2 diabetes mellitus in U.S. men. Ann Intern Med 2002; 136:201-209.

van Dam RM, Visscher AWJ, Feskens EJM: Dietary glycemic index in relation to metabolic risk factors and incidence of coronary heart disease: the Zutphen Elderly Study. Eur J Clin Nutr 2000;54:726-731.

van Lenthe FJ, van Mechelen W, Kemper HC, Post GB: Behavioral variables and development of a central pattern of body fat from adolescence into adulthood in normalweight whites: the Amsterdam Growth and Health Study. Am J Clin Nutr 1998;67:846852.

Vanselow MS, Pereira MA, Neumark-Sztainer D, Raatz SK: Adolescent beverage habits and changes in weight over time: findings from Project EAT. Am J Clin Nutr 2009;90:14891495.

van Wymelbeke V, Beridot-Therond ME, de La Gueronniere V, Fantino M: Influence of repeated consumption of beverages containing sucrose or intense sweeteners on food intake. Eur J Clin Nutr 2004;58:154-161.

Vartanian LR, Schwartz MB, Brownell KD: Effects of soft drink consumption on nutrition and health: a systematic review and meta-analysis. Am J Public Health 2007;97:667-675.

Vartiainen E, Puska P, Pietinen P, et al: Effects of dietary fat modifications on serum lipids and blood pressure in children. Acta Paediatr Scand 1986;75:396-401.

Ventura AK, Loken E, Brich LL: Risk profiles for metabolic syndrome in a nonclinical sample of adolescent girls. Pediatrics 2006;118: 2434-2442.

-Villegas R, Liu S, Gao YT, et al: Prospective study of dietary carbohydrates, glycemic index, glycemic load, and incidence of type 2 diabetes mellitus in middle-aged Chinese women. Arch Intern Med 2007;167:2310-2316.

Wakai K, Date C, Fukui M, et al: Dietary fiber and risk of colorectal cancer in the Japan collaborative cohorte study. Cancer Epidemiol Biomarkers Prev 2007;16:668-675.

Walter U, Schwartz FW: Prävention; in Schwartz FW, Badura B, Busse R, et al. (eds): Das Public Health Buch. Urban and Fischer Verlag. München, Jena 2003, pp S189-S214.

Wang L, Gaziano JM, Liu S, et al: Whole- and refined-grain intakes and the risk of hypertension in women. Am J Clin Nutr 2007;86: $472-479$.

Wannamethee SG, Whincup PH, Thomas MC, Sattar N: Associations between dietary fiber and inflammation, hepatic function, and risk of type 2 diabetes in older men: potential mechanisms for the benefits of fiber on diabetes risk. Diabetes Care 2009;32:18231825 .
Weijenberg MP, Mullie PF, Brants HA, et al: Dietary glycemic load, glycemic index and colorectal cancer risk: results from the Netherlands Cohort Study. Int J Cancer 2008;122: 620-629.

Wen W, Shu XO, Li H, et al: Dietary carbohydrates, fiber, and breast cancer risk in Chinese women. Am J Clin Nutr 2009;89:283289.

Whelton SP, Hyre AD, Pedersen B, et al: Effect of dietary fiber intake on blood pressure: a meta-analysis of randomized, controlled clinical trials. J Hypertens 2005;23:475-481.

WHO: Global health risks: Mortality and burden of disease attributable to selected major risks. World Health Organization Geneva, 2009.

WHO: Diet, nutrition and the prevention of chronic diseases. World Health Organisation (WHO), Technical Report Series 916, 2003.

Williams CL, Strobino BA: Childhood Diet, Overweight, and CVD Risk Factors: The Healthy Start Project. Prev Cardiol 2008;11: 11-20.

$\checkmark$ Williams DE, Knowler WC, Smith CJ, et al: The effect of Indian or Anglo dietary preference on the incidence of diabetes in Pima Indians. Diabetes Care 2001;24:811-816.

-Witteman JC, Willett WC, Stampfer MJ, et al: A prospective study of nutritional factors and hypertension among US women. Circulation 1989;80:1320-1327.

World Cancer Research Fund/American Institute for Cancer Research (WCRF/AICR): Food, nutrition, physical activity and the prevention of cancer: a global perspective. American Institute for Cancer Research, Washington DC, 2007.

Wu H, Dwyer KM, Fan Z, et al: Dietary fiber and progression of atherosclerosis: the Los Angeles Atherosclerosis Study. Am J Clin Nutr 2003;78:1085-1091.

Yano K, Rhoads GG, Kagan A, Tillotson J: Dietary intake and the risk of coronary heart disease in Japanese men living in Hawaii. Am J Clin Nutr 1978;31:1270-1279.

Zhang X, Albanes D, Beeson WL, et al: Risk of colon cancer and coffee, tea, and sugarsweetened soft drink intake: pooled analysis of prospective cohort studies. J Natl Cancer Inst 2010;102:771-783.

Zhang C, Liu S, Solomon CG, Hu FB: Dietary fiber intake, dietary glycemic load, and the risk for gestational diabetes mellitus. Diabetes Care 2006;29:2223-2230. 